University of Louisville

ThinkIR: The University of Louisville's Institutional Repository

Electronic Theses and Dissertations

8-2013

\title{
Synapses, spines, zinc and pathology of Alzheimer's disease.
}

Dmitry Familtsev 1985-

University of Louisville

Follow this and additional works at: https://ir.library.louisville.edu/etd

\section{Recommended Citation}

Familtsev, Dmitry 1985-, "Synapses, spines, zinc and pathology of Alzheimer's disease." (2013). Electronic Theses and Dissertations. Paper 420.

https://doi.org/10.18297/etd/420

This Doctoral Dissertation is brought to you for free and open access by ThinkIR: The University of Louisville's Institutional Repository. It has been accepted for inclusion in Electronic Theses and Dissertations by an authorized administrator of ThinkIR: The University of Louisville's Institutional Repository. This title appears here courtesy of the author, who has retained all other copyrights. For more information, please contact thinkir@louisville.edu. 
SYNAPSES, SPINES, ZINC AND PATHOLOGY OF ALZHEIMER'S DISEASE

by

Dmitry Familtsev

M.D., Omsk State Medical Academy, Omsk, Russia, 2008

\author{
A Dissertation \\ Submitted to the Faculty of the \\ School of Medicine of the University of Louisville \\ in Partial Fulfillment of the Requirements \\ for the Degree of \\ Doctor of Philosophy \\ Department of Anatomical Sciences and Neurobiology \\ University of Louisville \\ Louisville, Kentucky
}

August 2013 

SYNAPSES, SPINES, ZINC AND PATHOLOGY OF ALZHEIMER'S DISEASE

\author{
By \\ Dmitry Familtsev \\ M.D., Omsk State Medical Academy, Omsk, Russia, 2008
}

A Dissertation Approved on

$\underline{0.5 / 08 / 2013}$

(Date)

By the following reading Committee:

Martha E. Bickford, Ph.D., Dissertation Director

Radhika Vaishnav, Ph.D.

George D. Mower, Ph.D.

Nicholas Mellen, Ph.D.

Michal Hetman, M.D., Ph.D. 


\section{ACKNOWLEDGEMENTS}

During the course of these years while learning what it takes to be a scientist, it was proven to me so many times that with wise and caring guidance even the grayest days could turn sunny quicker. I believe, your life as a graduate student is only as good as your mentor is. And I could not ask for a better life during my graduate years. Besides being a successful scientist, wise teacher and a great advisor, Dr. Martha Bickford, first of all, manage to be one of the best persons I know. Without her caring mentorship, my scientific aspirations could have never seen the light of a day. Without her friendly support, it would hardly be possible sometimes to concentrate on the science due to the stresses of daily life. Without her incredible understanding, it just would not be possible for me to achieve my professional and personal goals. She knew exactly when I needed to be pushed a little in a gentle but the most effective way; yet, she knew even better when I needed to be encouraged. Working with Dr. Bickford was an irreplaceable life experience because she taught me how to be passionate about my work, how to be resilient and, yet, flexible, and, above it all, she taught me how to be a better human, humble and forgiving. With this dissertation the great chapter of my life is coming to its conclusion but I will always be grateful for the day I entered Dr. Bickford's lab and was allowed to stay there for the next few great years.

I would also like to thank my co-mentor, Dr. Robert Friedland, for his valuable insights into the Alzheimer's disease studies and financial support for conducting them. His unquenchable enthusiasm toward the unveiling the secrets of this mysterious illness 
was very contagious and he was the reason I became interested in the studying of Alzheimer's disease in the first place. My only regret is that I was not always able to consult him more often.

I am endlessly thankful for the great impact the members of my committee brought into my work. Dr. Radhika Vaishnav with her valuable experience in Alzheimer's disease has always given me a great advice on how to improve my research. Dr. Michal Hetman gave me a few useful hints, especially on western blot techniques and provided me with his lab space and resources to actually conduct a large portion of my western blot experiments. Dr. George Mower was always first to notice any of imperfections in my experiments and did not hesitate to describe a simple and robust way of addressing them. Dr. Nicholas Mellen, besides his insightful advices, showed me what the real passion for discovery means; he taught me how important it is to be able to move on quickly when something would not work without sacrificing the whole idea and search for the alternative ways instead. All of them were available for me whenever I needed their help and incredibly supportive.

I would like to thank Dr. Jeff Petruska and his whole team, especially Dr. Ben Harrison, who was a great teacher and a friend outside the lab. They taught me many great skills over the course of my education. Dr. Aruna Vashishta, Justin Hallgren from Dr. Hetman's lab for their time and knowledge sharing. I thank Dr. Suresh Tiyagi and Dr. Natia Qipshidze for making my life a little easier with the usage of imaging device for my western blot experiments. I would also give a special thanks to Darlene Burke for helping me with biostatistics part of this work.

I would also like to thank my lab colleagues, especially lab manager Arkadiusz Slusarczyk, for all that he taught me, and I talk not only about technical knowledge and chemistry. He was a great listener and a friend, his optimism was so influential that I could not resist but share it. My former lab members, Dr. Haiyang Wei, Dr. Jon Day- 
Brown, Donna Dillahay and Na Zhou were a great support and help. I would specifically thank Dr. Sean Masterson for being such a great friend for me during all this time. And of course, I would like to finish this acknowledgement with bending my knees in front of my loving wife, Anastasia Familtseva, who was endlessly understanding, supportive and caring, who gives a meaning for me to continue it all.

Thank you all, it was a pleasure and a great experience. 
This work is dedicated to my grandparents, the best any grandson could wish for. Without you I would still be just another guy whose dreams have not come true. 


\title{
ABSTRACT \\ SYNAPSES, SPINES, ZINC AND THE PATHOLOGY OF ALZHEIMER'S DISEASE
}

\author{
Dmitry Familtsev
}

May, $8^{\text {th }}, 2013$

\begin{abstract}
Alzheimer's disease (AD) is a multifactorial illness manifesting as gradual progressive memory loss, culminating in an end-stage of profound cognitive deterioration. While $\mathrm{AD}$ pathology is characterized by the presence of beta amyloid (A $\beta$ ) plaques, clearing $\mathrm{A} \beta$ deposits from the brain has not proved sufficient to improve cognition. It is thought that the gradual loss of synaptic connections in the telencephalon leads to cognitive impairment. Thus, understanding synaptic function and testing methods to prevent the loss of synapses are the primary directions of current $\mathrm{AD}$ research.
\end{abstract}

$\mathrm{AD}$ pathology in the primary sensory areas of the cortex (the granular or koniocortex) is typically found only in the most advanced cases. To gain insight into the differences in the synaptic organization of the koniocortex and the eulaminate cortex, we compared the distribution and morphology of geniculocortical and pulvinocortical terminals in tree shrews. Synaptic terminals were labeled using the stereotaxic injection of neuroanatomical tracers or viral vectors, and/or immunohistochemical labeling of the type 2 vesicular glutamate transporter (vGLUT2) and gamma aminobutyric acid (GABA). Transmitted light, confocal and electron microscopy revealed that geniculocortical 
terminals in layers IVa and IVb of the striate cortex are significantly larger than pulvinocortical terminals in the temporal cortex. Geniculocortical terminals contact nonGABAergic dendritic spines (91\%) and the GABAergic dendrites of cortical interneurons (9\%), while pulvinocortical terminals only contact nonGABAergic dendritic spines. Geniculocortical terminals often contain small extensions of postsynaptic spines, termed "spinules", while pulvinocortical terminals do not. Analysis of the postsynaptic targets of geniculocortical terminals revealed that $14 \%$ contained a spine apparatus, an organelle related to dendritic spine stability and memory. Our results indicate that the organization of thalamocortical synaptic connections is quite different in the konicortex and eulaminate cortex. Further comparisons of the synaptic organization of the koniocortex and eulaminate cortex may reveal characteristics related to the progression of $\mathrm{AD}$ pathology.

The brain areas primarily affected by $\mathrm{AD}$ are mirrored by the distribution of dendritic spines that contain spine apparati (uniquely identified by the actin-bindng protein synaptopodin) and by glutamatergic terminals that contain zinc ions sequestered by the type 3 zinc transporter ( $\left.\mathrm{ZnT}_{3}\right)$. Because zinc assists in the rapid aggregation of $\mathrm{A} \beta$, and zinc levels increase and decrease with brain activity, we examined levels of ZnT3 and synaptopodin in the cortex and hippocampus of a transgenic mouse model of $\mathrm{AD}$ (TgSwDI; this model expresses neuronally derived human amyloid $\beta$-precursor protein, APP gene, 770 isoform, containing the Swedish K670N/M671L, Dutch E693Q and Iowa D694N mutations, under the control of the mouse thymus cell antigen 1, theta, Thy1, promoter). Using western blot techniques we measured $\mathrm{ZnT}_{3}$ and synaptopodin levels in tissue from mice at 1, 4 and 6 months of age before and after zinc precipitation by intraperitoneal injection of sodium selenite. We found that sodium selenite treatment produced no significant effect on the levels of synaptopodin. However, we did find that ZnT3 levels were higher in TgSwDI mice at 4 months of age in both the cortex and 
hippocampus when compared to wild type mice (WT) and TgSwDI mice of other ages. Also, when compared by ages, synaptopodin levels were higher in 4-month old WT and TgSwDI animals in both the cortex and hippocampus. These results suggest that zinc may be an important participant in the pathology of $\mathrm{AD}$, but that age-related changes in ZnT3 levels should be considered when evaluating treatments involving the manipulation of zinc levels.

Finally, using electron microscopy and immunohistochemical labeling of activated microglia with isolectin b4 (Ib4), we investigate the histopathology of the cortex and hippocampus in TgSwDI mice. Introduction of Dutch/Iowa mutation caused a strong affinity of $\mathrm{A} \beta$ deposition near brain vasculature. We found that pathology in the brains of TgSwDI mice progresses in the same areal sequence as is seen in human $\mathrm{AD}$ patients and other AD mouse models. However, the cortex and hippocampus are largely devoid of neuritic plaques; the abundance of $\mathrm{A} \beta$ accumulation was observed in and around blood vessel walls surrounded by microglia cells. Our finding suggest the this mouse model of $\mathrm{AD}$ is very suitable for investigations of cerebral amyloid angiopathyrelated aspects of $\mathrm{AD}$. 
TABLE OF CONTENTS

PAGE

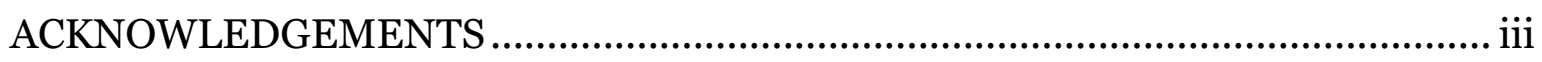

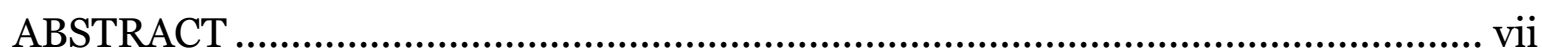

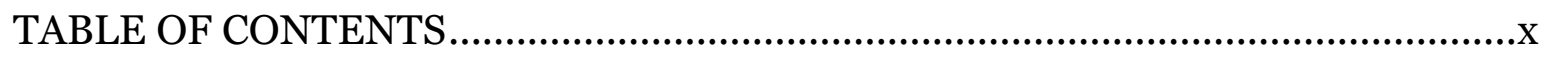

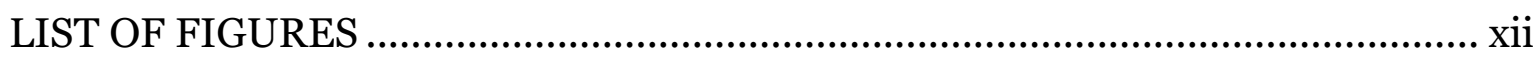

CHAPTERS

I. INTRODUCTION

II. ULTRASTRUCTURAL COMPARISON OF GENICULOCORTICAL AND PULVINOCORTICAL PROJECTIONS IN THE TREE SHREW .............................14

Introduction ......................................................................................... 14

Materials and Methods ............................................................................. 15

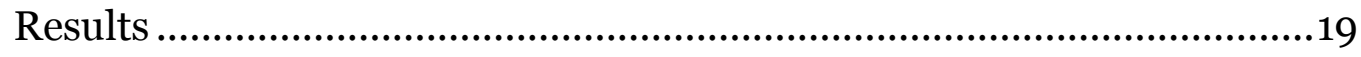

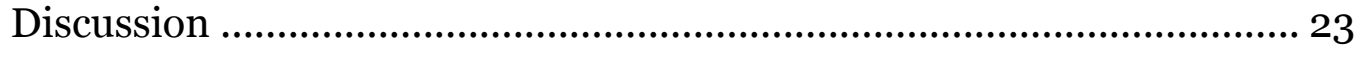

III. ALTERATION OF SYNAPTOPODIN AND ZINC TRANSPORTER TYPE 3 PROTEIN IN WILD TYPE AND TRANSGENIC MODEL OF ALZHEIMER'S DISEASE CONTAINING SWEDISH, DUTCH AND IOWA MUTATIONS

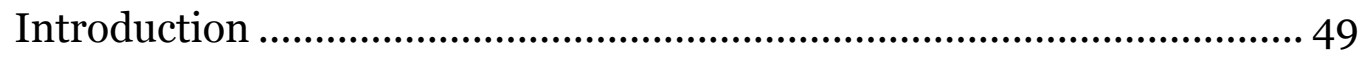

Materials and Methods ........................................................................... 55

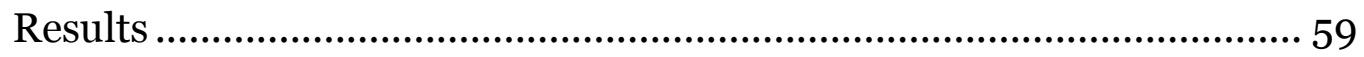

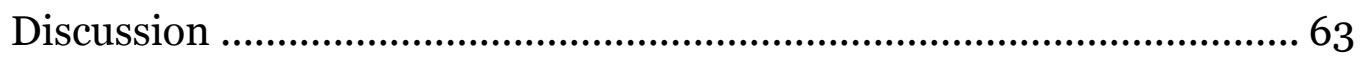

IV. BETA-AMYLOID PLAQUE FORMATION: HISTOCHEMICAL AND ULTRASTRUCTUTAL CHARACTERISTICS IN A TRANSGENIC MOUSE MODEL OF ALZHEIMER'S DISEASE CONTAINING SWEDISH, DUTCH AND IOWA 


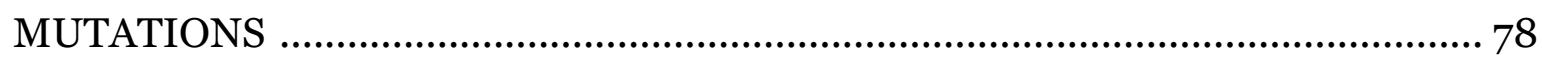

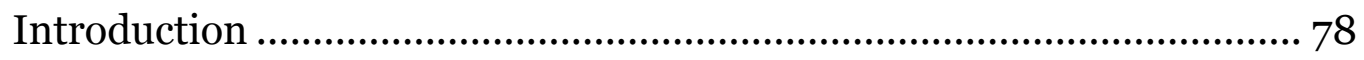

Materials and Methods ......................................................................... 83

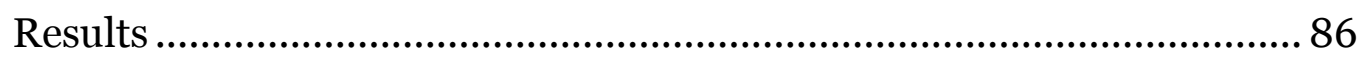

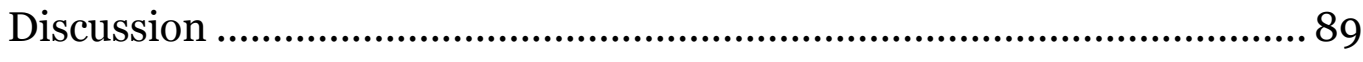

V. SUMMARY, THEORETICAL CONSIDERATIONS AND FUTURE

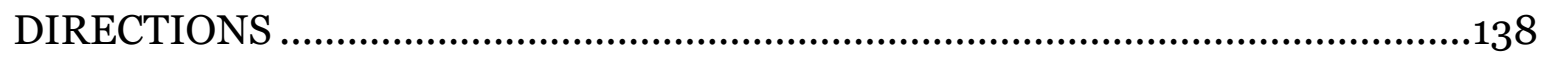

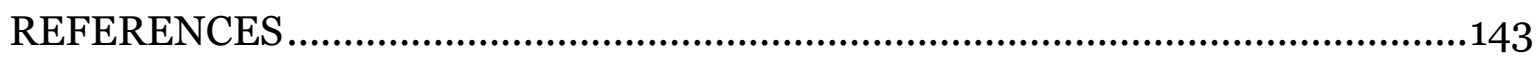

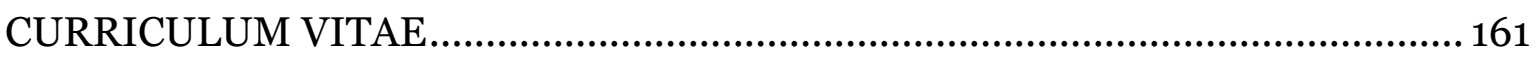




\section{LIST OF FIGURES}

FIGURE

PAGE

Chapter I

1.1 The hipoocampus anatomy

Chapter II

2.1 Schematic of dorsal lateral geniculate nucleus (dLGN) and pulvinar projections to the cortex

in tree shrew

2.2 The location of virus injection in the dLGN

2.3 The location of BDA tracer injections in the dLGN

2.4 Distribution and morphology of pulvinocortical terminals in the temporal cortex, and geniculocortical terminals in $\mathrm{V} 1$

2.5 Confocal image of TdTomato and vGLUT2 labeled terminals co-localization

2.6 Micrograph of geniculocortical terminal connection in layer IVa of V1

2.7 Mean size of geniculocortical terminals (GC) in layer IV sublayers of V1

2.8 GABA-content in synaptic targets of geniculocortical terminals (GC) in layer IV

sublayers of $\mathrm{V} 1$

2.9 Percentage of spine apprati-containing postsynaptic profiles in each type of

synaptic contact 
2.10 Comparison of pre- and postsynaptic sizes of geniculocortical (GC) versus pulvinocortial (PC) terminals

Chapter III

3.1 Zinc and synaptopodin staining of rat brain

3.2 Western blot bands from the cortex of 4-month old and 6-month old

3.3 Synaptopodin and $\mathrm{ZnT}_{3}$ levels before and after sodium selenite treatment in cortex and hippocampus of 1-month old WT and TgSwDI mice

3.4 Synaptopodin and $\mathrm{ZnT}_{3}$ levels before and after sodium selenite treatment in cortex and hippocampus of 4-month old WT and TgSwDI mice

3.5 Synaptopodin and ZnT3 levels before and after sodium selenite treatment in cortex and hippocampus of 6-month old WT and TgSwDI mice

Chapter IV

4.1 Confocal image of $\mathrm{A} \beta$ plaques and associated microglia in 13-month old TgSwDI

4.2 Confocal image of mossy fibers (MF) stained for A $\beta$ with $4 \mathrm{G} 8$ and

microglia with Ib4

4.3 Progression of AD pathology in TgSwDI at different ages

100

4.4 Sequential saggital sections of 20-month old TgSwDI stained for Ib4

102

4.5 IB4 staining in 20-month old TgSwDI

4.6 Tg2576 mature plaque core in 24-month old Tg2576

106

4.7 Mature plaque core with A $\beta$ fibrils, Tg2576 24-month old 


\section{CHAPTER I \\ INTRODUCTION}

Alzheimer's disease (AD) is characterized by progressive cognitive decline with the initial impairment of the ability to form new memories and then gradual involvement of all intellectual modalities. This eventually leads to the inability to perform the functions of daily life and premature death. $\mathrm{AD}$ is the most frequent cause of dementia in developed countries. In the United States there are approximately 5.5 million people affected, and it is estimated that worldwide there are 24 million people that have $\mathrm{AD}$ (Mayeux and Stern, 2012). The incidence and prevalence of $\mathrm{AD}$ is directly related to the age of the population. The incidence of $\mathrm{AD}$ rises exponentially after age 65 (Evans et al., 1989; Brookmeyer et al, 1998). However, about 5 percent of people affected by this disease have an early onset form, which is diagnosed when they are in their 40 and 50 s (Alzheimer's Disease Association).

Pathologically, both sporadic and early-onset $\mathrm{AD}$ is characterized by the same morphologic elements that include extracellular plaques of beta-amyloid and intracellular hyperphosphorylated tangles of the microtubule-associated protein tau (Braak and Braak, 1991; Perl, 2010). The latter is also called neurofibrillary tangles and they were described by Alois Alzheimer himself in his pioneering work as abnormal fibrous inclusions within the perikaryal cytoplasm of pyramidal neurons (Perl, 2010). Ultrastructural studies of neurofibrillary tangles reveal the helices of paired $10 \mathrm{~nm}$ fibrils of tau protein that are hyperphosphorylated in serine/threonine residues by kinases and have other attached constituents such as ubiquitin, cholinesterases and beta-amyloid 4 fibrils to them (Kidd, 1963; Wisniewski et al., 1976; Perry et al., 1987; Mesulam and 
Moran, 1987; Love et al., 1988; Trojanowski et al., 1991; Avila, 2006; Perl, 2010). Despite being a major histopathological factor for diagnosis of $\mathrm{AD}$, neurofibrillary tangles are not pathognomonic to this disease only and can be seen in other diseases of the central nervous system such as postencephalitic parkinsonism, posttraumatic dementia or dementia pugalistica, type C Niemann-Pick disease, and amyotrophic lateral sclerosis/parkinsonism dementia complex of Guam and others (Perl, 2010). The range of these diseases with their different etiologies, pathogeneses and clinical presentations could imply that neurofibrillary tangles are more of a consequence of different pathological processes affecting the neuronal tissue than the cause of them.

Another cardinal histopathological hallmark of $\mathrm{AD}$ is the senile plaque, or $\beta$ amyloid plaque, and it is also not totally unique to this disease. It can be seen in Lewy body dementia (Armstrong et al., 1997; Jendroska et al., 1997), Parkinson disease dementia (Jendroska et al., 1996), and others, even though studies succeeded in chemical and structural differentiation of the constituent beta-fibrils that form plaques in these etiologically diverse diseases (Wiltfang et al., 2002, Bibl et al., 2006). The core of the senile plaque is presented by the accumulation of a $4 \mathrm{kDa}$ protein with a betapleated sheet structure (Beyreuther et al., 1985; Tate et al., 1992). The beta-amyloid plaque consists of the central core of beta-amyloid fibrils, arranged in a radial fashion, and surrounding it neurites, that include dendrites or axons of dystrophic neurons, containing dense bodies, membranous profiles, and packets of paired helical filaments, as well as glial cells. Conglomerations of beta-amyloid fibrils, called "diffuse plaques", are also found and they are not surrounded by many dystrophic neurites; these diffuse deposits are often times found in the brains of older individuals and do not correlate with cognitive impairment (Kidd, 1964; Yamaguchi et al., 1989; Gentleman et al., 1989; Morris et al., 1996; Wegiel et al., 2000; Wegiel et al., 2004; Perl, 2010; Holtzman et al., 
2012). The ultrastructure of senile plaques is described in more detail in Chapter IV of this dissertation.

The distribution of plaques and tangles is a sequential event. The initial deposition starts within the phylogenetically oldest part of the cortex - the allocortex, which includes the olfactory bulb, entorhinal region, presubicular region, and hippocampal formation. As the disease progresses, plaques and tangles are found in the mesocortex, which includes the orbitofrontal region, cingluate gyrus and the parahippocampal gyrus. In the final stages of the disease, plaques and tangles are distributed in the neocortex. In all these areas, the affected neurons are those that form connections with other telencephalon areas. Telencephalic neurons with projections to the diencephalon, midbrain or hindbrain rarely develop neurofibrillary tangles (Braak et al, 2006). The distribution of amyloid plaques is related to the distribution of zinc in the brain, as well as the distribution of dendritic spines that contain a structure known as the spine apparatus. As described in more detail in Chapter III, we manipulated zinc levels in animal models to explore the role of zinc might play in $\mathrm{AD}$ progression (Arendash et al, 2004, Arendash et al., 2007; Costa et al., 2007, Harvey and Svoboda, 2007; Ashby and Isaac, 2011; Beique et al., 2012, Mundel et al., 1997; Deller et al., 2003; Vlachos et al., 2009, Paoletti et al., 2009).

The exact mechanism of $\beta$-amyloid formation is not clear, but the sequential proteolytic cleavages of ubiquitously expressed transmembrane polypeptide amyloidprecusor protein (APP) by metalloproteinases $\beta$ - and $\gamma$-secretases is implicated in the formation of $A \beta 40$ and $A \beta 42$ isoforms of beta-amyloid that are very capable of aggregation into the plaque-like structures. The formation of $\mathrm{A} \beta$ is physiological and occurs constantly in the normal brain. However, this process can become pathological depending on the way the APP is cleaved by secretases (reviewed by Selkoe, 2001; Pluta et al., 2013). Most forms of familial (early onset) AD are associated with mutations in 
genes involved in the processing of APP, but it is still unknown why the scales are shifted towards the formation of pathological beta-amyloid in the sporadic cases, and what causes its aggregation. The inheritance patterns of $\mathrm{AD}$ are not straightforward. The mutations in APP, presenilin-1 and presenelin-2 gene (PSEN-1 and PSEN-2) genes are mendelian forms and lead to altered structures of APP protein or $\gamma$-secretase activity that are ultimately responsible for increases in $\beta$-amyloid formation. The apolipoprotein $\mathrm{E} \varepsilon 4$ allele (APOE $\varepsilon 4)$ is more complex in terms of inheritance patterns. Being a proven risk factor for development of $\mathrm{AD}$, especially with two alleles present, not everyone with this allele will develop the disease. The latest genomic studies discovered at least 9 more risk loci for the development of $\mathrm{AD}$ (reviewed by Broeckhoven et al., 2013).

The discoveries of genetic mechanisms in $\mathrm{AD}$ pathology, have helped scientists to develop useful animal models for further investigations of disease. One such model is more thoroughly described in Chapters III and IV of this dissertation. However, earlyonset forms represent only the smallest fraction of $\mathrm{AD}$ cases, implying that there must be other mechanisms and pathways leading to excessive $A \beta$ formation and/or aggregation.

While, $\beta$-amyloid plaques are the most studied histopathological signs of $\mathrm{AD}$, it is still unknown whether they the main causative agents of the disease. Since 1992 when Hardy and Higgins first formulated their "amyloid cascade hypothesis" it was a predominant explanation of etiology and pathogenesis of Alzheimer's disease. According to this hypothesis the accumulation and assembly of $A \beta$ is a primary inciting event that leads to the formation of senile plaques, neuronal dystrophy and dementia (Hardy and Higgins, 1992; Reitz, 2012). Recent discoveries related to immunotherapy targeted against beta-amyloid in human phase III trials failed to show any improvements in the cognitive functioning of patients in the dementia stage of sporadic $\mathrm{AD}$ disease (Blennow et al., 2012; Sperling et al., 2012; Mullard, 2012; Aisen et al., 2012). Thus, $\beta$ amyloid may not be the sole driver of cognitive impairment per se, as previously thought 
(Hardy and Allsop, 1991; Hardy and Higgins, 1992; Jellinger, 2009). Rather, it is now recognized that a conglomeration of pathological changes throughout the whole brain may be responsible for the cognitive deterioration associated with $\mathrm{AD}$. $\beta$-amyloid deposition is always involved in $\mathrm{AD}$ development, but is clearly linked to other processes such as synaptic loss, neuronal degeneration and microglial activation (D'Andrea et al., 2004; Spires-Jones et al., 2009; Reddy et al.,2010; Mandrekar-Colucci and Landreth 2010; Spires-Jones and Knafo, 2012). $\beta$-amyloid deposition is now viewed not as the cause of $\mathrm{AD}$ but rather as just one of the pathogenetic factors of the disease. There is strong evidence of patients without cognitive deterioration presenting with $\beta$-amyloid deposition (Tomlinson et al., 1968; Terry et al., 1991; Bennett et al., 2006; Schneider et al., 2007). The poor correlation between the burden of plaques and tangles and cognition has been shown in several studies (Tyas et al., 2007; Haroutunian et al., 2008; Iacono et al., 2009; Savva et al., 2009; Ewbank and Arnold, 2009). These discoveries stress the multifactorial nature of sporadic AD. The failure of immunotherapy to improve cognition even after the significant reduction in $\beta$-amyloid plaque burden emphasized the importance of understanding the events that occur as this neurodegenerative disease progresses. Early detection with the possibility of early intervention and prevention of this progression has emerged as a primary goal in current studies of $\mathrm{AD}$ (Aisen et al., 2013).

This dissertation consists of the three separate chapters presented in a sequence that best describes the brain structures and mechanisms that I studied in an attempt to understand the basics of Alzheimer's pathology. In Chapter II I describe a study of the ultrastructure of synaptic connections using the visual system as a model. In this Chapter I will provide detailed descriptions of the relationships between presynaptic terminals, dendritic spines and spine apparati, the morphological hallmarks of synaptic plasticity and memory function (Deller et al., 2003; Dunaevsky and Harms, 2007; Beique et al., 
2012). With synaptic loss being a major correlate of cognitive deterioration in $\mathrm{AD}$ (DeKosky and Scheff, 1990; Terry et al., 1991; Spires-Jones et al., 2013) we felt it is necessary to study normal terminal connections to better understand their pathology in $\mathrm{AD}$.

Chapter III presents experiments designed to explore possible mechanisms of the early stages of $\mathrm{AD}$ development. It has been reported that people exercising higher levels of cognitive functions during their lifetime have a decreased chance of developing $\mathrm{AD}$, and this is further, supported by studies of animal housed in enriched versus deprived environments (Arendash et al, 2004, Arendash et al., 2007; Costa et al., 2007). Because increased zinc levels have been detected in sensory deprived brain regions (Brown and Dyck, 2002; Dyck et al., 2003; Land and Shamalla-Hanna, 2002), and zinc is abundant in senile plaques (Lovell et al., 1998; Lee et al., 1999; Adlard et al., 2008), we tested the hypothesis that zinc levels may be related to the synaptic loss seen in early stages of AD. (Spires-Jones and Knafo, 2012).

Finally, in Chapter IV, we took a closer look at the development and ultrastructure of $\mathrm{AD}$ pathology using a transgenic mouse model. In particular, the involvement of microglia during the different stages of disease was investigated as it is still a point of scientific debate whether glial cells involvement in $\mathrm{AD}$ is beneficial or deleterious (Mandrekar-Colucci and Landreth, 2010; Mrak, 2012; Aguzzi et al., 2013).

To facilitate understanding of the experiments described in Chapters II-IV, several brain structures are described below.

\section{Hippocampus}

Figure 1.1 displays major anatomic features of hippocampus. The pointed wedge of the hippocampus is the dentate gyrus that is characterized by tightly packed layers of granule cells. Underlying the dentate gyrus, is the first area of hippocampus proper, CA4, that is continuous with zones CA3 to CA1. These areas are characterized by a layer of 
pyramidal cells. After CA1 there is a zone called the subiculum, followed by the enthorhinal cortex (Amaral and Lavenex, 2006). External inputs come from layer 2 stellate cells of the enthorhinal cortex; their axons form the perforant path and terminate onto granule cells of the dentate gyrus and pyramidal cells of CA3. In addition, layer 3 of the enthorhimal cortex sends axons to CA1. Granule cells of dentate gyrus convey passing through them neocortical representations by their axons to CA3 and these fibers called mossy fibers ( Steward and Scoville, 1976; Claiborne et al., 1986; Acsady et al., 1998). From here, pyramidal cells of CA3 send axons to CA1, called Schaffer fibers. In turn, pyramidal cells of CA1 send their axons to the subiculum and deeper layers of enthorhinal cortex. The subiculum also sends its axons to layer $\mathrm{V}$ of the enthorhinal cortex, closing the loop (Andersen et al., 1971).

\section{Mossy Fibers}

The hippocampus is one of the first brain areas affected by amyloid pathology in AD (Braak and Braak, 1991). Mossy fibers (MF), which originate from the granule cells of the dentate gyrus, are seen to contain very abundant levels of zinc when stained with specific protocols (Frederickson et al., 2000; Paoletti et al., 2009). As reviewed by Barrionuevo et al, 2000, a single unmyelinated mossy fiber axon originates from each granule cell. This axon immediately gives rise to an extensive set of collaterals providing input to the polymorphic neurons of the hilus, the area of dentate gyrus beneath the granule cell layer that is rich in interneurons (Nicoll and Schmitz, 2005). Mossy fiber axons form synapses with excitatory and inhibitory cells of the hilus and area CA3. Even though hilar cells are mostly inhibitory interneurons, each of MF axon's 140-150 synapses makes about 10 connections with excitatory hilar cells. In CA3 the majority of MF synaptic connections are on interneurons and some are on pyramidal cells (Claiborne et al., 1986; Acsady et al., 1998). The subset of these interneurons in CA3 layer (called stratum lucidum) is spiny cells that contain glutamate (Soriano and 
Frotscher, 1993); these cells are considered local circuit excitatory cells (Barrionuevo et al., 2000). This type of interneuron is complemented by another, aspiny type of interneuron in $\mathrm{CA}_{3}$, that provides feedforward and feedback inhibition of MF activity (Vida and Frotscher, 2000; Lawrence and McBain, 2003). Thus, the large terminals of MFs contact CA3 pyramidal cells and hilar mossy cells, the principal cells in dentate gyrus hilus. Small terminals and filopodial extensions target GABA-containig neurons in the dentate hilus and stratum lucidum of CA3 (Nicoll and Schmitz, 2005). Through these complicated and still poorly understood connections, the hippocampus participates in the formation of new memories, a process that involves changes in dendritic spines.

\section{Dendritic spines}

A dendritic spine is a small protrusion from the dendritic shaft of pyramidal neurons in the neocortex and hippocampus, medium spiny neurons of the striatum, and other types of neurons. Filopodia are thin, hair-like protrusions that are precursors of dendritic spines (Fiala et al., 1998). Dendritic spines are classified in three major categories based on morphology: thin, stubby and mushroom. Thin and mushroom spines have a neck with a bulbous head whereas stubby spines are devoid of a neck. The head of mushroom spines is larger than that of thin spines (Jones and Powell, 1969; Peters and Kaiserman-Abramoff, 1970; Rochefort and Konnerth, 2012). The same spine can change its shape rapidly through activity-dependent or independent mechanisms (Harris and Stevens, 1989; Kasai et al., 2010). The majority of excitatory synapses occur on the dendritic spines (Bourne and Harris, 2007). Typically, glutamateric synaptic connections on spine heads are described as asymmetric synapses; this is because the presynaptic density is smaller than the postsynaptic density (Rochefort and Konnerth, 2012). It has been suggested that spine morphology determines the function of its synapse (Yuste et al., 2000; Kasai et al., 2010) and that the induction of long-term potentiation (LTP) of postsynaptic potentials elicited by the 
presynaptic terminal correlates with spine enlargement (Matsuzaki et al., 2004). In adult hippocampus and neocortex only about $25 \%$ of spines are mushroom-shaped. They can persist for months (Holtmaat et al., 2005). These larger spines can anchor more Nmethyl D-aspartate (NMDA) receptors and a-amino-3-hydroxy-5-methyl-4-isoxazole propionic acid (AMPA) glutamate receptors to make synapses functionally stronger (Matsuzaki et al., 2001; Ganeshina et al., 2004; Ashby et al., 2006; Bourne and Harris, 2007). These features suggest that mushroom spines are more stable 'memory spines' (Kasai et al., 2003; Bourne and Harris, 2007). It has been shown that the induction of LTP increases spine head size (Lang et al., 2004; Matsuzaki et al., 2004; Kopec et al., 2006), whereas long term depression (LTD) decrease spine size (Zhou et al., 2004) or causes spine retraction (Nagerle et al., 2004).

The mushroom spines are associated with the spine apparatus organelle, which is composed of stacked smooth endoplasmic reticulum (Vlachos, 2012). A marker for the location of spine apparati, and an essential component of spine apparati is synaptopodin, an actin-binding protein (Mundel et al., 1997; Deller et al., 2003). Synaptopodindeficient mice do not form spine apparati and show deficits in LTP and spatial learning (Bas Orth et al., 2007; Deller et al., 2007; Jedlicka et al., 2008; Vlachos, 2012). Since there is evidence of impaired spine stability in Alzheimer's disease (Spires-Jones et al., 2007; Spires-Jones and Knafo, 2012; Knafo et al., 2012) we considered it worthwhile to investigate the above mentioned structures more closely in this dissertation.

\section{Visual system}

The primary visual pathways of the brain transmit information from the retina, to the dorsal lateral geniculate nucleus (dLGN) to the primary visual cortex (V1), or striate cortex From V1, visual signals are transmited in two general streams, dorsal and ventral. The dorsal stream conveys information to middle temporal areas (also known as MT or V5) and the inferior parietal cortex. This stream is referred as "where" or "how" 
pathway, because it represents visual motion, object location and movement control. The ventral stream conveys information through area V4 to the inferior temporal lobe. This pathway is called the "what pathway" and is associated with form recognition and object representation and the storage of long-term memory (Ungerleider and Mishkin, 1982; Goodale and Milner, 1992; Ungerleider, 1995). Pathways through V1 are referred to as the primary pathways because lesions of V1 generally lead to an absence of visual perception. However, secondary visual pathways from the retina to the superior colliculus to thalamic nuclei such as the pulvinar nucleus also exist. These pathways are thought to be responsible for the responses to visual stimuli complete that can exist in the absence of perception (Stoerig and Cowey, 1997; Collins et al., 2003; Collins et al., 2005; Danckert and Rossetti, 2005). The interconnections of the superior colliculus, the pulvinar nucleus and the cortical and subcortical targets of the pulvinar nucleus are thought to participate in directing visual attention and visually guided movement (Chomsung et al., 2010).

There are two general types of thalamic neurons: relay cells and interneurons. The relay neurons send axons to the cortex and/or subcortical targets such as the amygdala and corpus striatum (Day-Brown et al., 2010). Relay cells are glutamatergic. The interneurons project locally within the thalamus and contain gamma aminobutyric acid (GABA) (Paletsini et al., 1993). Chapter II of this dissertation deals with the anatomy of the synaptic connections made by the dLGN in V1 and by the pulvinar nucleus in the temporal cortex.

The neurons of the neocortex can be divided into two broad categories: spiny excitatory cells (approximately 80\% of the total neuronal population) and smooth inhibitory cells (approximately 20\%) (Brown and Hestrin, 2009). There are 2 major classes of excitatory neurons in the cerebral cortex, including V1. The pyramidal cells are the principle source of efferent cortical axons (Lund et al., 1975; Katz 1987; Buhl and 
Singer, 1989; Hubener et al., 1990; Einstein and Fitzpatrick, 1991). Their interlaminar and collateral horizontal connections provide synaptic contact with other cortical neurons (Gilbert and Wiesel, 1979, 1983; Martin and Whitteridge, 1984; Carder and Hendry, 1994) in layers II, III, V and IV, creating the basic circuit across different areas of cortex (Feldman, 1984; Martin and Whitteridge, 1984; Carder and Hendry, 1994). These pyramidal cells are found to be gutamatergic (Carder and Hendry, 1994) and zincergic as well (Brown and Dyck 2005; Miyashita et al., 2007). Pyramidal cells are found in all cortical areas. Spiny stellate cells have only been described within the koniocortex (or granular cortex) i.e. regions of the cortex that contain densely packed cells in layer IV. Within these cortical areas, which includes V1, the spiny stellate cells are restricted to layer IV where they receive thalamocortical innervation. From there, spiny stellate cells can send some axons into other areas of the cortex, but the majority form exclusively intracortical connections with neurons in the superficial layers (Lund, 1973; Carder and Hendry, 1994). Interneurons can be differentiated by anatomical, physiological and molecular criteria (for review, Petilla Interneuron Nomenclature Group, 2008). They form complex networks in neocortex by selective chemical and electrical synapses on pyramidal cells and other interneurons playing a major role in shaping neocortical activity (Brown and Hestrin, 2009). This inhibition may improve orientation sensitivity, spatial frequency selectivity and specifying the spatial position of an object's border (Hirsch et al., 2003).

In summary, this work developed as the attempt to reveal certain pathogenetic principles in the progression of Alzheimer's disease, with a parallel deeper look into the structural organization of the cortex that could be altered by this disease. 
Figure 1.1 A. The hippocampus anatomy. (A) Pointed wedge represents dentate gyrus, granule cells of which form mossy fibers. Mossy fibers relay information to hilus and CA3 area of hippocampus, form where through the series of axonal connections information is relayed to $C A 2, C A 1$ and subiculum, scale $=100 \mu \mathrm{m}$. (B) Schematic of hippocampal anatomy and connectivity depicted by Cajal (1911). Arrows demonstrate the fiber connectivity of the trisynaptic pathway in the hippocampus. In this circuit, entorhinal cortex (ent) neurons innervate dentate granule neuron dendrites via the perforant pathway. Granule cell axons, called mossy fibers, then project to CA3 pyramidal neurons. A diffuse projection of CA3 axons forms the Schaffer collateral system innervating the CA1 strata radiatum and oriens. Finally, axons from CA1 and CA3 project via the alveus to the fimbria. The orientation and localization of these pathways define anatomical layers within the hippocampus $[1=$ subiculum, 2 = perforant pathway axons, 3 = molecular layer, 4 =granule cell layer, $5=$ hilum, $6=$ mossy fiber axons, $7=C A 3$ pyramidal neurons, $8=$ Schaffer collateral axons, $9=$ stratum lacunosum - moleculare, $10=$ stratum radiatum, $11=C A 1$ pyramidal neurons, 12 = stratum oriens, $13=$ dorsal hippocampal commissure, $14=$ alveus, 15 = fimbria; $D G$ - dentate gyrus] Figure and legend adopted and modified from Shepherd et al., 2006 

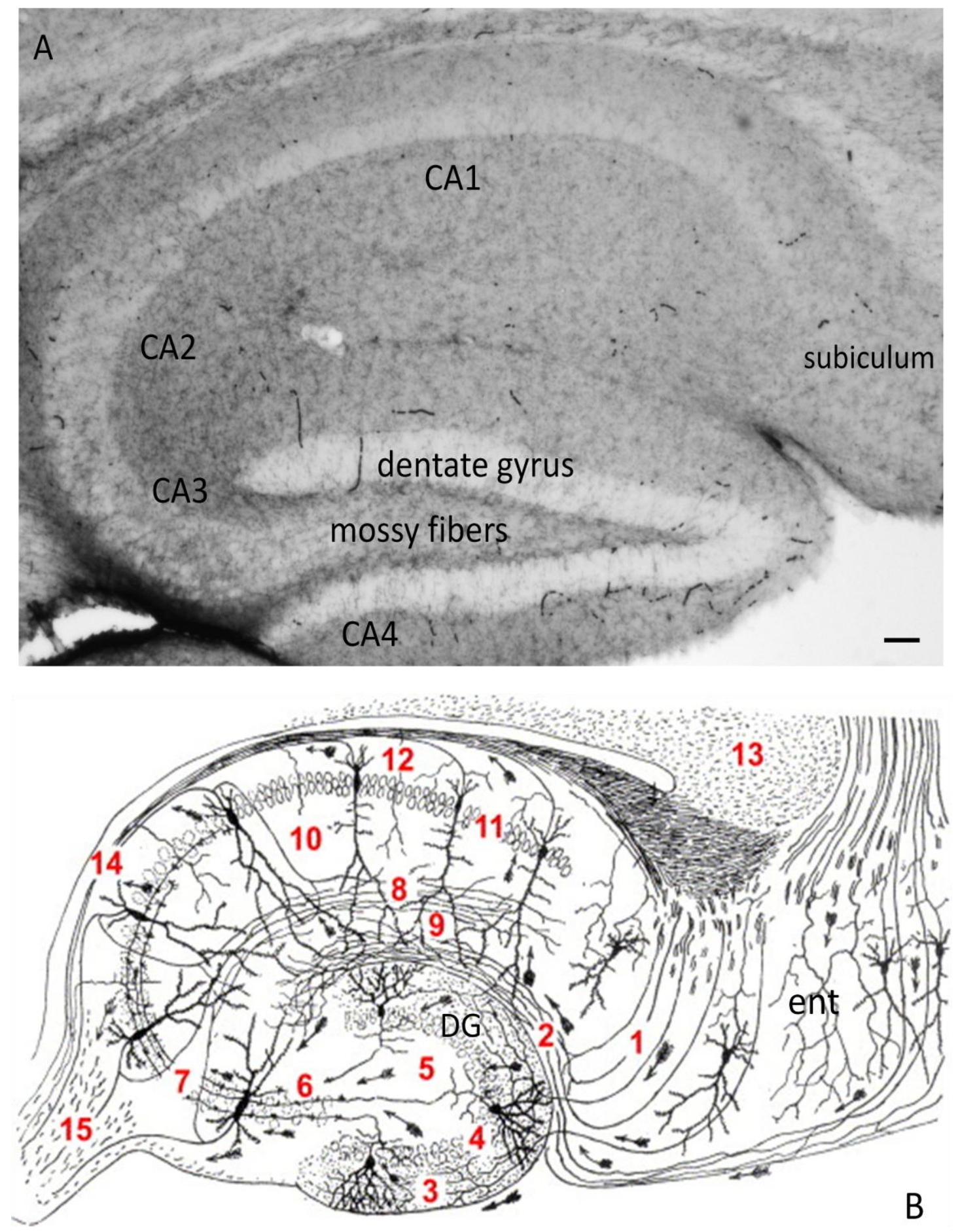


\section{CHAPTER II}

\section{ULTRASTRUCTURAL COMPARISON OF GENICULOCORTICAL AND PULVINOCORTICAL PROJECTIONS IN THE TREE SHREW}

\section{Introduction}

The striate cortex is referred to as koniocortex or granular cortex because layer IV contains a population of small densely packed neurons, many of which can be identified as spiny stellate cells (Douglas et al 2004). Studies in primates have shown that geniculocortical terminals primarily contact spiny stellate cells, and additionally contact non-spiny interneurons (Hendrickson et al., 1981; Peters and Payne, 1993; da Costa and Martin, 2011). The extrastriate visual cortex is referred to as eulaminate or homotypical cortex because the cell size and packing density within layer IV is not distinctly different from that found in other layers. This suggests that spiny stellate cells are not common in this region of the cortex, and further suggests that the organization of thalamocortical synapses is likely to be significantly different in granular versus homotypical cortical areas. Our previous study of the projections from the pulvinar nucleus to the temporal cortex in the tree shrew (Tupaia) revealed that pulvinocortical terminals innervate layers I-IV, where they all contact dendritic spines (Chomsung et al., 2010). The goal of the current study was to examine the ultrastructure of geniculocortical terminals in the tree shrew so that we could directly compare the characteristics of thalamocortical terminals in the striate and extrastriate cortex of the same species.

Although now classified in the order Scadentia, tree shrews are still considered to represent a prototype of early prosimian primates. This is because the tree shrew visual system exhibits many of the characteristics of the primate visual system, including a 6 
layered dorsal lateral geniculate nucleus (dLGN) (Kaas and Preuss 1993; Kaas 2002). Layers 1 and 4 of the tree shrew dLGN receive input from the ipsilateral retina, while layers 2 and 5 receive input from the contralateral retina. Layers 3 and 6 receive input from the superior colliculus (Diamond et al., 1980, 1981, 1982). Layers 1, 2, 4, 5 and 6 project to layer IV of the striate cortex, while layers 3 and 6 project to layers I-III. Layer IV is divided into two sublayers IVa and IVb. IVa sublayer receives inputs from dLGN layers 1, 2 whereas IVb receives projections from layers 4 and 5 (Conley et al., 1984). In this way, both subtiers of layer IV receive information from the ipsi- and contralateral retina (Figure 2.1).

\section{Materials and Methods}

A total of 5 adult tree shrews (Tupaia belangeri) were used for the experiment. One adult tree shrew was used to label geniculocortical terminals in the area IVb of primary visual cortex (V1) by anterograde transport. The injection of adenoassociated virus (AAV) coupled to fluorescent protein TdTomato in the layer 1 of ipsilateral dorsal geniculate nuceus (dLGN) was used (TS 12-01V). Selected sections were stained with antibody against TdTomato (Living Colors ${ }^{\circledR}$ DsRed polyclonal antibody made in rabbit, 1:1000, Clontech) or the type 2 vesicular glutamate transporter (vGLUT2, made in guinea pig, 1:10ooo, Chemicon Temecula, CA) and embedded for electron microscopy (EM). The data about labeled thalamocortical terminals by anterograde transport with biotinylated dextran amine (BDA) injection (300o MW; Molecular Probes, Eugene, OR) in the pulvinar nucleus was collected by previous investigators and used for this experiment as described before by Chomsung et al., 2010. In short, 4 tree shrews received an injection of BDA in the pulvinar nucleus. All the methods used were approved by the University of Louisville Animal Care and Use Committee and conform to the National Institutes of Health guidelines. 


\section{Injection of virus and BDA}

Tree shrews that received AAV carrying a vector for channelrhodopsin 1 and 2 fused to tdTomato (Vector Biolabs, serotype 2/1, titer $=2.0 \times 1012 \mathrm{VG} / \mathrm{ml}$ ) and BDA injection were anesthetized with intramuscular injection of ketamine $(100 \mathrm{mg} / \mathrm{kg})$ and xylazine $(6.7 \mathrm{mg} / \mathrm{kg})$ mixture. As needed, additional supplements of that mixture were administered to assure deep anesthesia until the injection procedure was complete. The tree shrews were placed into a stereotaxic apparatus and prepared for sterile surgery. Using scalpel, the vertical cut of the skin overlying the skull was done along the middle line and the skull exposed for perforation. A small area of the skull overlying dLGN (TS 12-01V) and pulvinar nucleus (4-05-5, 5-06-6AB, 8-06-1D, 10-04-1AB) was removed and the dura reflected. The pressure injection was made using 34 gauge needle attached to a nanofil syringe; an utlramicropump UMP-4 was used to eject $100 \mathrm{nl}$ at a rate of 30 $\mathrm{nl} / \mathrm{min}$. For BDA, a glass pipette with tip diameter of $3 \mu \mathrm{m}$ filled with $\mathrm{BDA}$ in $5 \%$ saline was lowered vertically and BDA injected ionophoretically using positive $2 \mu \mathrm{A}$ current for 30 minutes.

\section{Immunohistochemistry}

Several antibodies were used in the experiment. Some sections from tree shrew injected with virus was stained with anti-DsRed antibodies. After the tree shrew survived for 7 days, it was given a lethal dose of ketamine $(600 \mathrm{mg} / \mathrm{kg})$ and xylazine (130 $\mathrm{mg} / \mathrm{kg}$ ) and was perfused with Tyrode solution, followed by a fixative of $2 \%$ paraformaldehyde and 2\% glutaraldehyde in 0.1M phosphate buffer, $\mathrm{pH} 7.4$ (PB). After the brain was removed from the skull, it was sectioned using a vibratome with a thickness of $50 \mu \mathrm{m}$ and placed in PB. To reveal virus labeled terminals, sections were incubated with antibody against TdTomato (Living Colors $®$ DsRed polyclonal antibody made in rabbit, 1:1000, Clontech). To reveal BDA, section were incubated in a 1:100 dilution of avidin and biotinylated horseradish peroxidase (ABC; Vector Laboratories, 
Burlingame, CA) in phosphate-buffered saline (o.01 M PB with 0.9\% NaCL, pH 7.4; PBS) with $1 \%$ normal goat serum (NGS) overnight at $4^{\circ} \mathrm{C}$. After that the sections were rinsed in $\mathrm{PB}$ and reacted with nickel-intensified 3,3'-diaminobenzidine (DAB) for 5 minutes and subsequently washed in PB 3 times. After the third wash, sections were mounted on slides for light microscope examination or were prepared for electron microscopy (as described by Chomsung et al., 2010).

Other sections from this animal were stained with antibody against the vGLUT2 (1:10000) made in guinea pig (Chemicon Temecula, CA) to reveal geniculocortical terminals. For that, after preincubating the sections in 10\% NGS and 0.1\% Triton X-100 in PBS for 30 minutes, they were placed in solution containing anti-vGLUT2 antibodies and incubated overnight at $4^{\circ} \mathrm{C}$. After rinsing the sections in $\mathrm{PB}$ they were placed in a 1:100 dilution of biotinylated goat anti-guinea pig IgG (Vector Laboratories, Burlingame, CA) in $1 \%$ NGS/PBS for 1 hour at room temperature. The sections were rinsed in PB three times and subsequently reacted with nickel-enhanced DAB for 5 minutes. After thorough rinse in $\mathrm{PB}$, the sections were prepared for electron microscopy.

After sections were prepared, cut and mounted for electron microscopy (described below), they were stained for GABA by using previously reported techniques (Patel and Bickford, 1997). For this, the GABA antibody made in rabbit (Sigma Chemical Company, Saint Louis, MO) in concentration of 1:2000 were tagged with a goat-antirabbit antibody conjugated to $15 \mathrm{~nm}$ gold particles (Amersham, Arlington Heights, IL).

For double-labeling of terminals tissue injected with virus was stained for antivGLUT2 antibodies (1:1000o) with secondary anti-guinea pig AlexoFluor-488conjugated antibodies (1:2000) (Invitrogen). After washing the sections in PB 3 times, they were mounted on slides using ProLong® Gold antifade agent (Invitrogen). After coverslipping the slides they were ready to be viewed under the confocal microscope. 


\section{Electron microscopy}

To prepare tissue for electron microscopy, selected sections that contained terminals labeled with DAB to reveal the location of BDA, TdTomato or vGLUT2 were postfixed in $2 \%$ osmium tetroxide, dehydrated in ethyl alcohol series and preincubated in Durcupan resin overnight in a vacuum chamber. The next day, these sections were flat embedded between two sheets of Aclar plastic (Ladd Reasearch, Williston, VT). Using a light microscope, the sections were examined to select areas of interest. Selected areas were mounted on reisn blocks and ultrathin sections $(70-80 \mathrm{~nm})$ were cut using a diamond knife. Ultrathin sections were collected on Formvar-coated nickel slot grids. Selected ultrathin sections were stained for GABA as described above. After air drying the sections were stained with a 10\% solution of uranyl acetate in methanol for 30 minutes and examined with an electron microscope.

\section{Ultrastructural data analysis}

Prepared ultrathin sections were examined using a Phillips CM10 electron microscope equipped with a digital camera (SIA-12C). Images of labeled terminals that were involved in synaptic contacts were captured at a magnification of $18000 \mathrm{X}$. Using Maxim DL (C) 5 software the area of the pre- and postsynaptic profiles was measured. The presence or absence of spine apparati, and spinules were noted and the gold particle density within pre- and postsynaptic profiles was calculated. The ultrastructural features of adjacent terminals were also noted. The postsynaptic profiles were considered to be GABAergic if the gold particle density was at least 2 times the density found in the presynaptic thalamocortical terminals. For statistical analysis of size differences an independent t-test was used. 


\section{Results}

\section{Distribution and morphology of geniculocortical and pulvinocortical terminals}

Figure 2.2 illustrates the location of virus injection in the dLGN. The BDA injection was limited to the most lateral layers of the dLGN - layers 5 and 6 (figure 2.3). After the virus injection, TdTomato expression was primarily limited to the most medial layer of the dLGN -layer 1. Confirming previous reports (Fitzpatrick, 1996) the BDA injection labeled geniculocortical terminals in layer IVb and III of V1 while the virus injection labeled terminals in layer IVa of V1. The labeled geniculocortical axons exhibited beaded boutons along their length with single axons extending horizontally and parallel to the cortical surface in layer IVa or IVb (figure 2.3, 2.4). In contrast, pulvinocortical axons in the temporal cortex (described in Chomsung et al. 2010), extend vertically from layer IV to layer I, with beaded axons oriented perpendicular to the cortical surface (Figure 2.4).

\section{Geniculocortical terminal vGLUT2 content}

To investigate whether geniculocortical terminals contain vGLUT2, we incubated sections that contained TdTomato-labeled geniculocortical terminals with the antivGLUT2 antibody and tagged this antibody with Alexafluor 488. Examination of this tissue with a confocal microscope revealed that many TdTomato-labeled geniculocortical terminals were labeled with the vGLUT2 antibody (white areas in Figure 2.5). To quantify the colocalization of TdTomato and vGLUT2, we used ImageJ software to calculate the percent TdTomato-labeled axons (purple + white) that contained vGLUT2 (white). We only analyzed areas that included both TdTomato and vGLUT2 labeling to account for limitation in antibody penetration. Three images of $0.5 \mu \mathrm{m}$ scan thickness were analyzed. Using this method, we calculated that $19.63 \pm 1.6 \%$ of the TdTomato- 
labeled axons contain vGLUT2. This is comparable to previous studies of geniculocortical terminals using these methods (Nahmani and Erisir, 2005). The relatively low percentage of double-labeling reflects the fact that vGLUT2 is only contained within synaptic terminals and is absent from portions of axons that do not contain vesicles (Nahmani and Erisir; 2005; Chomsung et al., 2008).

\section{Synaptic targets of geniculocortical terminals in the sublayer IVa of visual} cortex

We examined the synaptic targets of 100 geniculocortical terminals in layer IVa labeled by adenovirus injection into the layer 1 of dLGN and 106 geniculocortical terminals labeled with the anti-vGLUT2 antibody in layer IVa of the same tree shrew. The size of the geniculocortical terminals labeled by the anti-vGLUT2 antibody (mean area $\left.1.23 \pm 0.34 \mu \mathrm{m}^{2}\right)$ was found to be significantly $(\mathrm{p}<.001)$ larger than the size of terminals labeled from adenovirus injections in layer 1 of the dLGN (mean area $0.93 \pm$ $0.54 \mu \mathrm{m}^{2}$ ).

The geniculocortical terminals labeled by either method formed asymmetric contacts with spines and small dendrites of similar sizes (mean area of profiles postsynaptic to TdTomato-labeled terminals was $0.36 \pm 0.2 \mu \mathrm{m}^{2}$, mean area of profiles postsynaptic to vGLUT2-labeled terminals was $0.34 \pm 0.2 \mu \mathrm{m}^{2}, \mathrm{p}=.2$ ). The majority of these synaptic contacts were classified as simple, i.e. the terminal and postsynaptic profile were connected by a synaptic cleft with one continuous postsynaptic density ( $72 \%$ of the TdTomato-labeled terminals made simple contacts and 60\% of the vGLUT2labeled terminals made simple contacts). The remaining synapses were classified as perforated (two separate postsynaptic densities were identified in the postsynaptic profile; $25 \%$ of synapses made by TdTomato-labeled terminals, $33 \%$ of synapses made by vGLUT2-labeled terminals) or multiple (more than two separate postsynaptic densities 
identified; $3 \%$ of synapses made by TdTomato-labeled terminals, $7 \%$ of synapses made by vGLUT2-labeled terminals).

The examined sections were stained for GABA as described in the methods section to evaluate whether geniculocortical terminals contact GABAergic cells. The density of gold particles overlying pre- and postsynaptic profiles was calculated to classify profiles as GABAergic or nonGABAergic. With the presumption that geniculocortical terminals are non-GABAergic (Chomsung et al., 2009), the gold particle density overlying presynaptic profiles was considered to represent background staining. Postsynaptic profiles were considered to be GABA-positive if they displayed a gold particle density at least two times greater than that of the adjacent presynaptic profile. According to this criterion, the majority of profiles postsynaptic to labeled geniculocoritecal terminals were non-GABAergic (91\% of profiles postsynaptic to TdTomato labeled terminals and $92.5 \%$ of profiles postsynaptic to vGLUT2-labeled terminals). Representative electron micrographs are illustrated in Figure 2.6.

\section{Comparison of the synaptic targets of geniculocortical terminals in layer IVa} and $\mathrm{IVb}$

We compared the ultrastructure of vGLUT2-labeled terminals in layer IVa and $\mathrm{IVb}$ in tissue from the same animal. We compared 106 synapses in sublayer IVa and 105 synapses in sublayer IVb. We found that vGLUT2-labeled terminals in sublayer IVa were significantly larger than vGLUT2-labeled terminals in IVb (mean area $1.23 \pm 0.34 \mu \mathrm{m}^{2}$ in IVa, mean area $1.0 \pm 0.53 \mu \mathrm{m}^{2}$ in IVb, $\left.\mathrm{p}=.002\right)$. The terminals in both sublayers formed asymmetric synapses with spines and small dendrites of comparable size $\left(0.34 \pm 0.2 \mu \mathrm{m}^{2}\right.$ in IVa and $0.32 \pm 0.15 \mu \mathrm{m}^{2}$ in IVb, $\mathrm{p}=.03$ ). The majority of synaptic contacts were simple (60\% simple, 33\% perforated and 7\% multiple in IVa and $71 \%$ simple, $26 \%$ perforated and $3 \%$ multiple in IVb). Lastly, the majority of postsynaptic dendrites contacted by the 
labeled terminals were non-GABAergic in both sublayers (92.5\% in IVa and $90.5 \%$ in IVb). The results are summarized by the graphs in Figs 2.7 and 2.8 .

\section{Ultrastructural characteristics of the postsynaptic targets of geniculocortical terminals}

Because the presence of a spine apparatus is correlated with spine volume as well as the amplitude of postsynaptic potentials generated by the terminals presynaptic to them (Vlachos et al., 2009; Segal, 2010), we quantified the size of labeled geniculocortical terminals and their postsynaptic targets that contained spine apparati and compared them to the size of labeled geniculocortical terminals and their postsynaptic profiles without spine apparati. We found that postsynaptic targets with clearly identifiable spine apparati comprised only $14 \%$ of all targets contacted by labeled geniculocortical terminals (total $n=311$ ). Spine apparati were only found in nonGABAergic profiles. We found only slight differences in the size of nonGABAergic profiles contacted by labeled geniculocortical terminals that did or did not contain spine apparati (mean area of postsynaptic targets with spine aparati $0.37 \pm 0.17 \mu \mathrm{m}^{2}, \mathrm{n}=43$; mean area of postsynaptic targets without spine apparati $0.33 \pm 0.17 \mu \mathrm{m}^{2}, \mathrm{n}=241$; $\mathrm{p}=.053)$. The size of the labeled terminals contacting profiles with spine apparati (1.078 $\pm 0.59 \mu \mathrm{m}^{2}$ ) was almost identical to the size of terminals contacting profiles lacking spine apparati $\left(1.073 \pm 0.59 \mu \mathrm{m}^{2}, \mathrm{p}=.48\right)$.

We also examined the types of synapses made by geniculocortical terminals on profiles with and without a spine apparatus and found that contacts on spines that contained a spine apparatus were more complex (54.5\% formed perforated synapses and 11.5\% formed multiple contacts) than contacts on spines that did not contain a spine apparatus. When calculated relative to each type of geniculocortical synaptic contact, only $7 \%$ of all simple contacts were formed with spines that contained a spine apparatus, 
whereas approximately one third of all perforated (31\%) or multiple (36\%) synapses were formed with spines that contained a spine apparatus (figure 2.9).

Erisir and Dreusicke, 2005, noted that geniculocortical terminals in the ferret contain small protrusions "spinules" that are extending from spines and embedded inside the terminal bouton (figure 2.6). We found that $58 \%$ of geniculocortical terminals labeled by AAV contained spinules, whereas nearly none of pulvinocortical terminals and geniculocortical terminals labeled by vGLUT2 contained them.

\section{Comparison of the synaptic targets of geniculocortical terminals and pulvinocortical terminals}

We compared the features of the 311 geniculocortical synapses examined in this study to 444 pulvinocortical synapses examined in the previous study from our laboratory (Chomsung et al, 2010). The size of geniculocortical terminals (mean area $\left.1.06 \pm 0.58 \mu \mathrm{m}^{2}\right)$ was significantly $(\mathrm{p}<.001)$ larger than the size of pulvinocortical terminals $\left(0.51 \pm 0.28 \mu \mathrm{m}^{2}\right)$. The postsynaptic targets contacted by geniculocortical terminals were also significantly $(\mathrm{p}<.001)$ larger (mean area $\left.0.34 \pm 0.19 \mu \mathrm{m}^{2}\right)$ than those contacted by pulvinocortical terminals $\left(0.28 \pm 0.11 \mu \mathrm{m}^{2}\right)$. Pulvinocortical synapses were as a group more complex (51\% simple, $35 \%$ perforated, $14 \%$ multiple) than geniculocortical terminals ( $71 \%$ simple, $28 \%$ perforated and $4 \%$ multiple). Finally, pulvinocortical terminals did not contact any GABAergic profiles whereas $9 \%$ of geniculocortical terminals contacted GABAergic dendrites. These results are summarized in figure 2.10.

\section{Discussion}

\section{Comparison with previous studies of geniculocortical terminals}

The distribution of geniculocortical terminals labeled by our tracer and adenovirus injections confirm previous tree shrew studies (reviewed by Fitzpatrick 
1996). We found that an injection in layer 1 of the dLGN labeled terminals in sublayer IVa of V1 and an injection sin layer 5 and 6 of the dLGN labeled terminals in layer IVb and III of V1. The labeled geniculocortical axons formed arbors that are oriented parallel to the cortical surface, similar to the orientation of the dendrites of spiny stellate cells (Muly and Fitzpatrick, 1992).

The ultrastructure of the labeled geniculocortical terminals, as well as terminals labeled with an antibody against vGLUT2, was similar to previous description of geniculocortical terminals in the mouse, ferret, cat and primate (Garey and Powell, 1971; Freund et al., 1989; Dehay et al., 1991; Erisir and Dreusick, 2005; Nahmani and Erisir, 2005; Coleman et al., 2010; daCosta and Martin, 2011). Most previous studies have described geniculocortical terminals as large terminals that primarily contact dendritic spines. Our results also are comparable to previous studies that have examined the percentage of contacts on GABAergic and nonGABAergic dendrites. Erisir and Dreusicke (2005) found that $9 \%$ of geniculocortical terminals in the adult ferret contact GABAergic dendrites, which is precisely the percentage of contacts on GABAergic dendrites revealed in our study. Freund et al (1989) found that 4.5-9.5\% of geniculocortical terminals in the adult macaque monkey contact GABAergic dendrites.

We also confirmed geniculocortical terminals in the tree shrew contain vGLUT2, as has been found in the ferret (Nahmani and Erisir, 2005) and mouse (Coleman et al., 2010). Not all geniculocortical axons were double-labeled with the vGLUT2 antibody, but this can be attributed to limitations in antibody penetration as well as the fact that vGLUT2 is limited to regions of the axons that contain synaptic vesicles. We did find that terminals labeled by the vGLUT2 antibody in layer IVa were larger as a population compared to the terminals labeled by the adenovirus injection in dLGN layer 1 . The fact that the same animal was used for quantifying all terminal types eliminates the possibility that fixation differences can account for any size differences. One explanation 
could be that geniculocortical terminals that originate from dLGN layer 2 are larger than those that originate from layer 1 . Alternatively, vGLUT2 may be more densely distributed in larger terminals, which contain greater numbers of vesicles, and immunocytochemical detection may be skewed toward larger terminals. However, vGLUT2-labeled terminals in layer IVb were smaller than the vGLUT2-labeled terminals in layer IVa. This could potentially be related to the size of cells in the various geniculate lamina. Brauer et al (1983) found that cells in layer 1 and 2 of the tree shrew dLGN are larger than cells in layers 4 and 5 , and therefore may give rise to larger terminals.

\section{Spines and spinules}

Spine apparati are composed of stacked smooth endoplasmic reticulum and found only in most mature dendritic spines of the hippocampus (Segal, 2010; Vlachos, 2012). This organelle together with its uniquely associated protein synaptopodin, is thought to participate in the long-term potentiation of synapses and spatial learning (Deller et. al., 2003, 2007; Jedlicka et al., 2008, 2009). It has been shown that in cultures of the mouse hippocampus the heads of the spines containing spine apparati were larger than spines that did not contain a spine apparatus (Vlachos et al., 2009). To investigate whether this also holds true for spines postsynaptic to geniculocortical terminals, we measured area of the postsynaptic spines that did or did not contain a spine apparatus. Only $14 \%$ of all geniculocortical targets contained a spine apparatus. All were nonGABAergic, as previously described by Freund et al. (1989). The size of postsynaptic spines that contain a spine apparatus was slightly larger than the size of spines that did not contain spine apparati, but this difference was not found to be significant. Likewise, there was no significant difference in the size of the terminals that contacts spines with or without a spine apparatus. However, we did find that synapses associated with spine apparati were more complex (perforated or multiple synapses) than synapses on spines that did not contain a spine apparatus. Thus, although future 
studies are necessary to determine how spine apparati are related to synaptic function, the presence of spine apparati in the visual cortex may be associated with differences in the distribution of postsynaptic receptors and the resulting strength of synaptic connections, as has been found in the hippocampus.

We also found the presence of spinules in tree shrew geniculocortical terminals, as was reported by Erisir and Dreusick (2005) in the ferret and Freund et al., 1989 in the adult monkey's V1. We found that $58 \%$ of all geniculocortical terminals had spinules in them. Although, the origin and formation process of spinules is not well understood, it has been shown that their formation is induced by heightened synaptic activity with their possible role in adjusting synaptic size in face of intense activity (Tao-Cheng et al., 2009).

\section{Comparison of geniculocortical terminals in the on and off}

\section{sublamina}

To investigate whether there are any differences in geniculocortical terminals related to the organization of the on and off sublamina of the visual cortex, we compared vGLUT2-labeled terminals in layer IVa and IVb. We found that geniculocortical terminals and their postsynaptic targets were significantly larger in layer IVa, when compared to layer IVb. As discussed above, this could be related to finding that cells in the dLGN layers 1 and 2 are larger than cells in dLGN layers 4 and 5 (Brauer et al., 1983). We also found that more geniculocortical synapses in layer IVa were complex (perforated or multiple, 40\%) when compared to geniculocortical synapses in layer IVb (29\%). Finally, we found similar numbers of synapses on GABAergic profiles in layer IVa (7.5\%) and IVb (9.5\%). Thus, while there were subtle differences in the geniculocortical synapses within layer IVa and IVb, there were no obvious structural differences that would differentially influence the receptive field properties in these sublamina. Freund et al., (1989) came to a similar conclusion after comparing the ultrastructure of 
geniculocortical axons originating from the magno and parvocellular layers of the primate dLGN.

\section{Comparison of geniculocortical and pulvinocortical terminals and}

\section{their synaptic targets}

We compared the data collected in the present study to that collected in a previous study of pulvinocortical connections (Chomsung et al., 2010). We found that geniculocortical terminals and their postsynaptic profiles are significantly larger when compared to pulvinocortical terminals and their postsynaptic profiles (figure 2.9). We also found that geniculocortical terminals contain abundant spinules (as in Freund et al 1989; Erisir and Dreuskicke, 2005), but pulvinocortical terminals contain none. This suggests that spinules may be a structure that is unique to spiny stellate cells. However, a similar morphology has been described for corticocortical terminals in area MT ( $\left.\mathrm{V}_{5}\right)$ that originate from V1 (Anderson et al., 1998), even though spiny stellate cells have not been described in this region of the cortex. It has been suggested that one function of the elaborate morphology of terminals with embedded spinules may be to secure efficient and reliable transmission (Anderson et al., 1998). In this regard, it is of interest that the response properties in area MT have been shown to decrease dramatically after lesions of V1, whereas pulvinar lesions have a smaller effect (Leiby et al., 1982; Bender and Butter, 1987). In the hippocampus, spinules have also been described in the dentate gyrus (Tarrant and Routtenberg, 1977) and CA1 region (Spacek and Harris, 1998) and the number of spinules increases in response to synaptic activity (Applegate and Landfield, 1988; Schuster et al., 1990). Therefore, spinules could indicate terminals that exhibit a higher level of activity.

We also found that the synapses formed by pulvinocortical terminals are more complex (perforated or multiple) than those formed by geniculocortical terminals, and whereas pulvinocortical terminals do not contact any GABAergic profiles, approximately 
$9 \%$ of geniculocortical terminals contact GABAergic profiles. Finally, we found that the horizontal orientation of layer IV geniculocortical terminals is quiet distinct from the more vertically oriented pulvinocortical terminals. Thus, future studies are necessary to determine the functional distinction between these projections, our results clearly suggest that the organization of thalamocortical synapses are quite different in the konicortex and eulaminate cortex. 
Figure 2.1 Schematic of dorsal lateral geniculate nucleus (dLGN) and pulvinar projections to the cortex in tree shrew. $d L G N$ consists of 6 layers. Layers 1 and 4 of the tree shrew $d L G N$ receive input from the ipsilateral retina, while layers 2 and 5 receive input from the contralateral retina. Layers 1, 2, 4, 5 and 6 project to layer IV of the striate cortex, while layers 3 and 6 project to layers I-III. Layer IV is divided into two sublayers IVa and IVb. IVa sublayer receives inputs from dLGN layers 1, 2 whereas IVb receives projections from layers 4 and 5 . The $d L G N$ projections to Iva arise in layers 1 and 2 and relay input from $\mathrm{ON}$-center ganglion cells, whereas projections to IVb come from layers 4 and 5 and receive their retinal input from OFF-center ganglion cells. Pulvinocortical terminals innervate layers I-IV of the temporal cortex. Pc - central pulvinar nucleus; Pd-dorsal pulvinar nucleus 


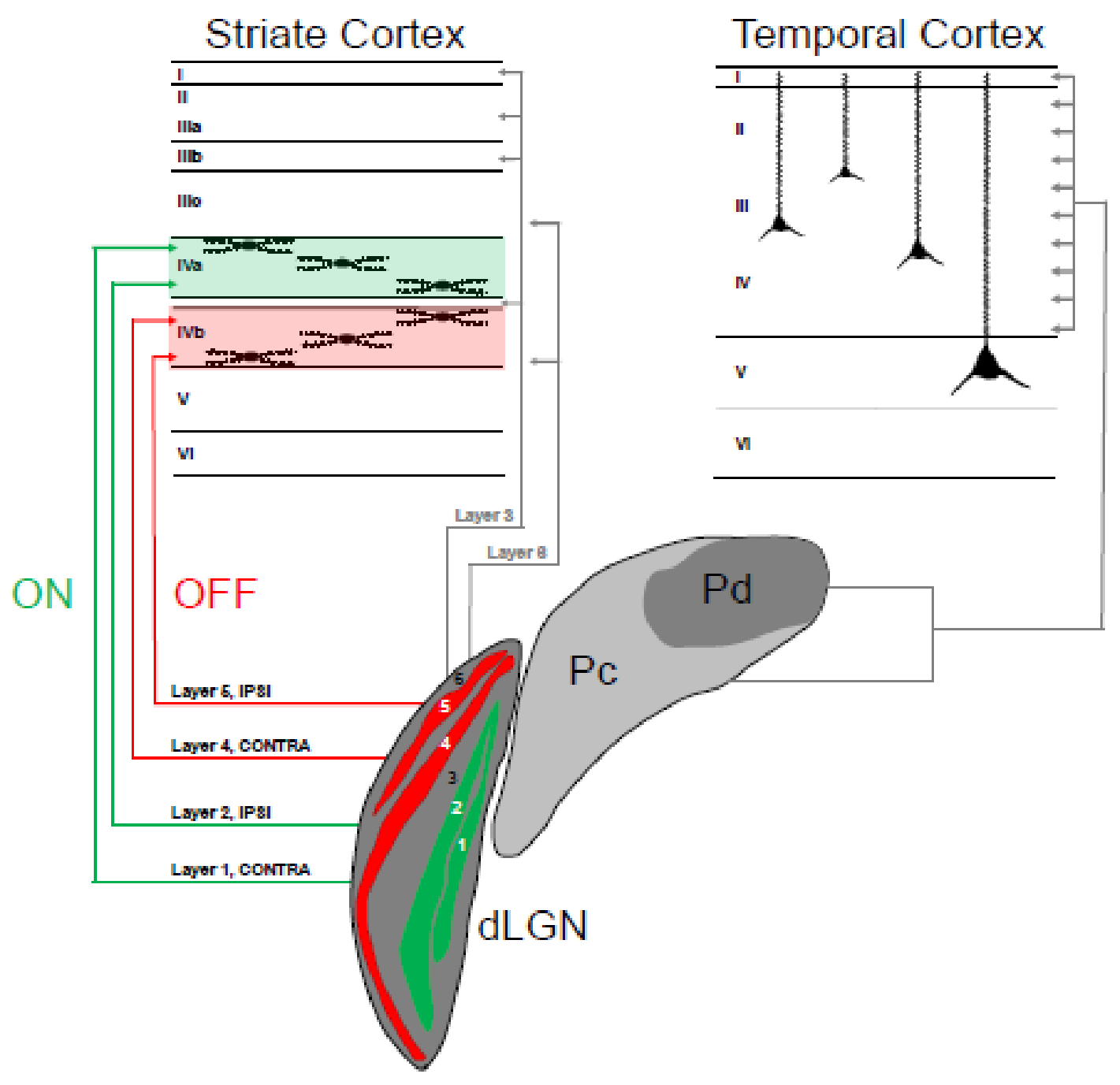


Figure 2.2 The location of virus injection in the dLGN. Expression TdTomato (red) in $d L G N$ cells and terminals after an AAV injection in layer 1 of the $d L G N$. The tissue was stained for vGLUT2 (green). (A) After the virus injection, TdTomato expression was primarily limited to the most medial layer of the dLGN-layer 1 and labeled terminals in layer IVa of V1 by anterograde transport (B). vGLUT2-containing geniculocortical terminals are seen only in layer IV of V1; areas labeled by both TdTomato and vGLUT2 are seen in yellow. Scale bar in $(B)=100 \mu \mathrm{m}$ and also applies to $(A)$. 


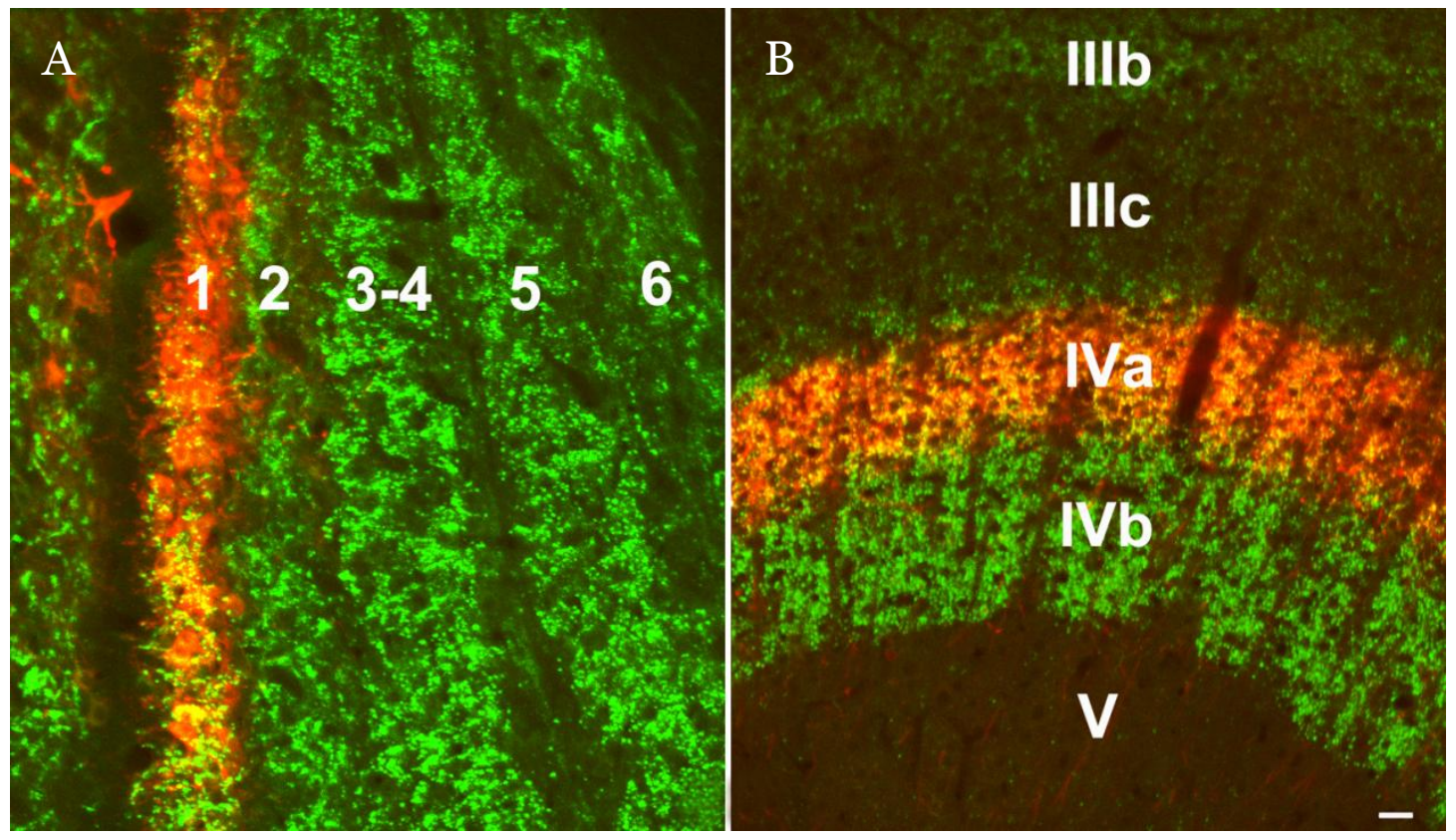


Figure 2.3 The location of BDA tracer injections in the dLGN. (A) BDA injection site in layer 5-6 of dLGN, PUL - pulvinar; OT - optic tract; scale bar = 25O $\mu \mathrm{m}(\mathrm{B})$ Distribution of geniculocortical terminals labeled by BDA in layer IVb and sparsely in III. Geniculocortical terminals are distributed in distinct laminae oriented parallel to the striate cortex surface. (C) BDA labeled terminals after injection in layer 5 labeled geniculocortical terminals in layer IVb and III of V1. The layers of V1 are numbered I through $V$. Scale bar in $(C)=1 \mathrm{~mm}$ 

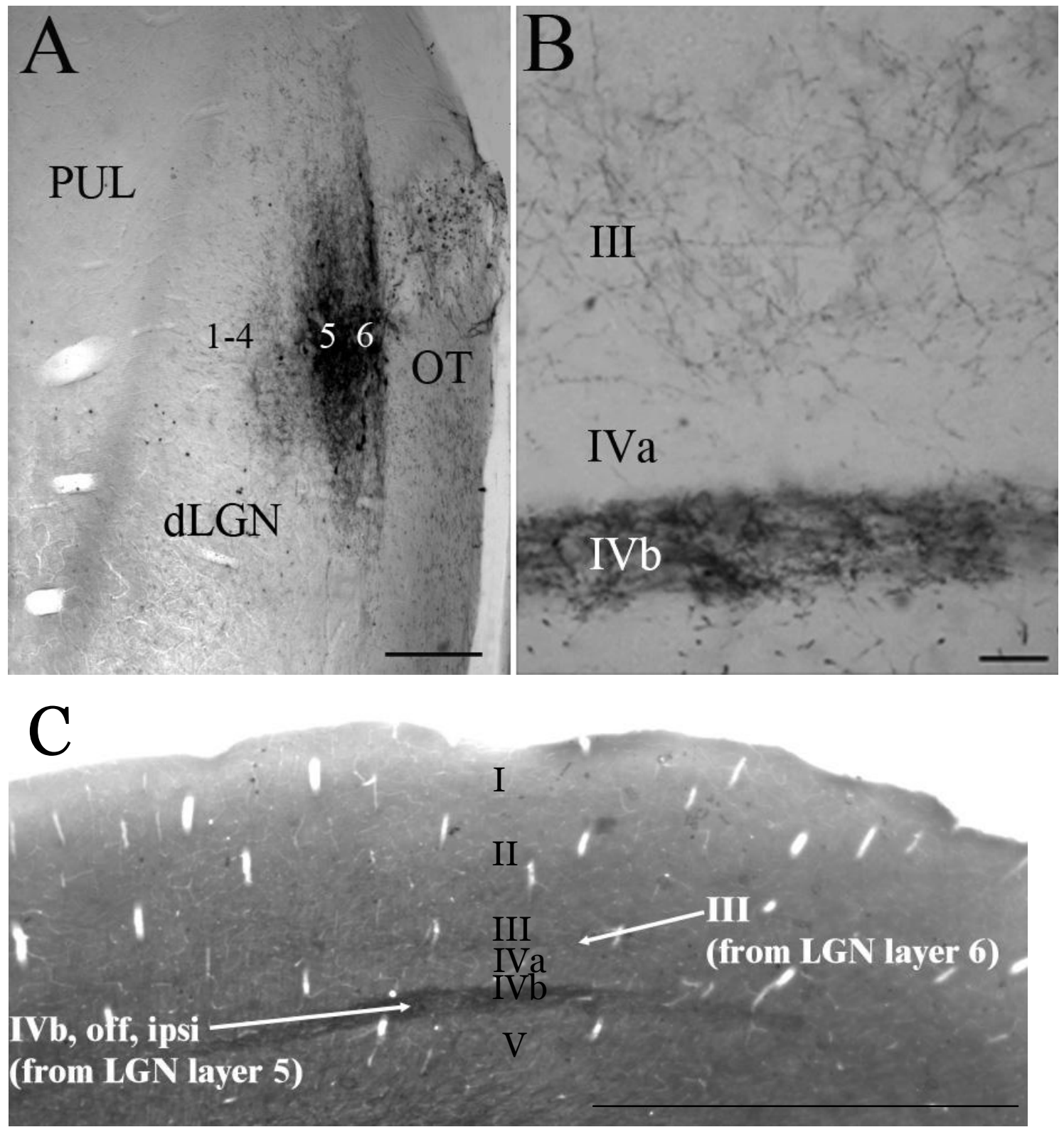
Figure 2.4 The micrographs illustrate the distribution and morphology of pulvinocortical terminals in the temporal cortex, and geniculocortical terminals in V1. Geniculocortical terminals are distributed in distinct laminae oriented parallel to the striate cortex surface (A), while pulvinocortical terminals are mostly oriented perpendicular to the temporal cortex surface (B). Geniculocortical terminals form dense terminal clusters in layer IV $(C)$, while pulvinocortical terminals are distributed along the length of axons that span layers $I V-I(D)$. Scale bar in $A=100 \mu \mathrm{m}$ and also applies to $B$. Scale bar in $C=30 \mu \mathrm{m}$ and also applies to $D$ 


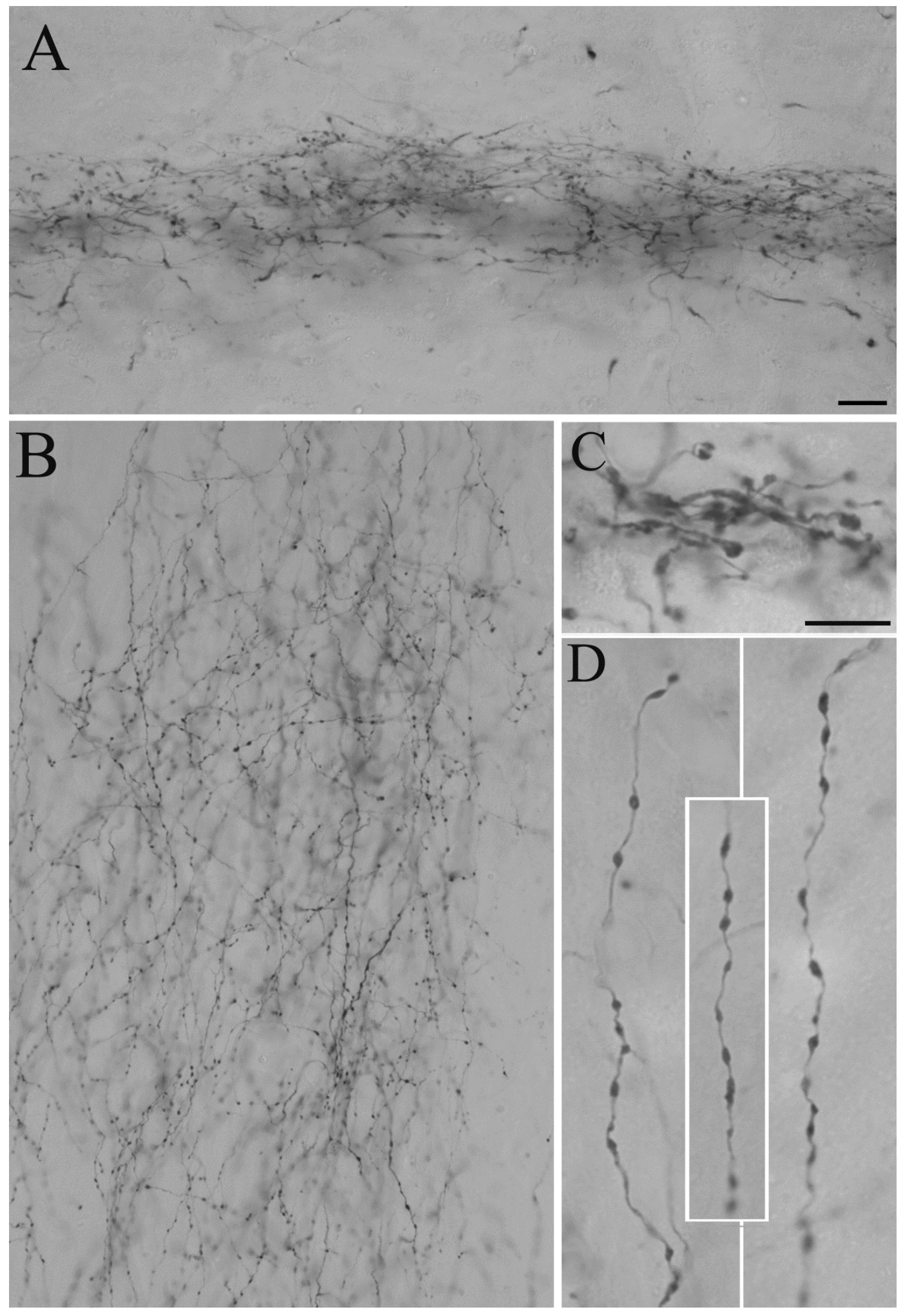


Figure 2.5 Confocal image of TdTomato and vGLUT2 labeled terminals co-localization. (A) Terminals containing $v G L U T 2$ are shown in green. (B) Terminals labeled by TdTomato are shown in purple. (C) Shows co-localization of terminals from (A) and (B) in white. (D) Higher magnification example of co-localized terminals (white) labeled by vGLUT2 (green) and TdTomato (purple). $19.63 \pm 1.6 \%$ of the TdTomato-labeled axons contained vGLUT2. Scale bar $=10 \mu \mathrm{m}$ and applies to (A), (B), (C) and (D). 


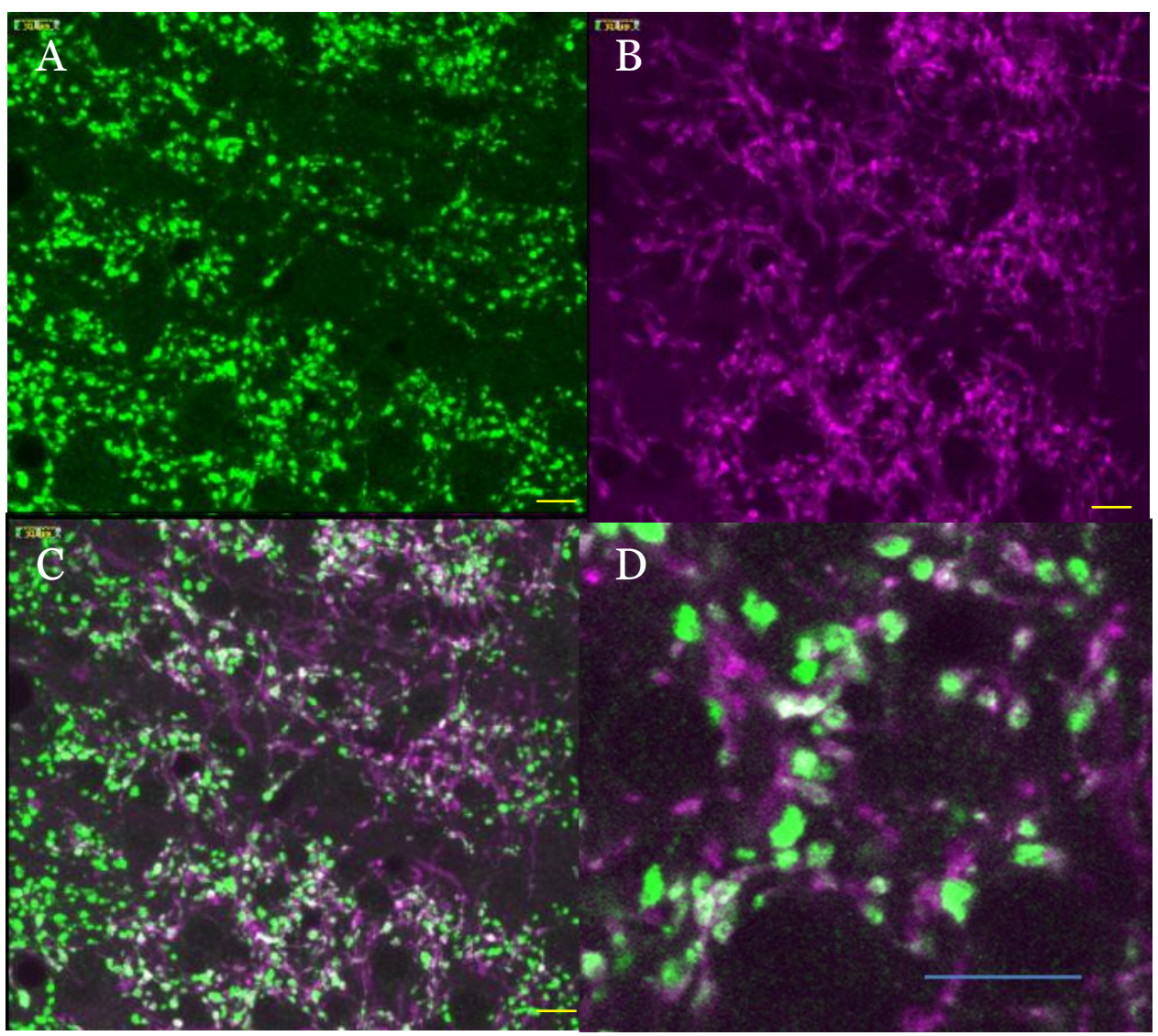


Figure 2.6 Micrograph of geniculocortical terminal connection in layer IVa of V1. Geniculocortical terminals $(A)$ are larger than pulvinocortical terminals $(B, C)$ and contain spinules (asterisks) that are not found within pulvinocortical terminals. Both primarily contact (white arrows) nonGABAergic dendritic spines that occasionally contain a spine apparatus (black arrow). Scale bar $=0.5 \mathrm{um}$. 

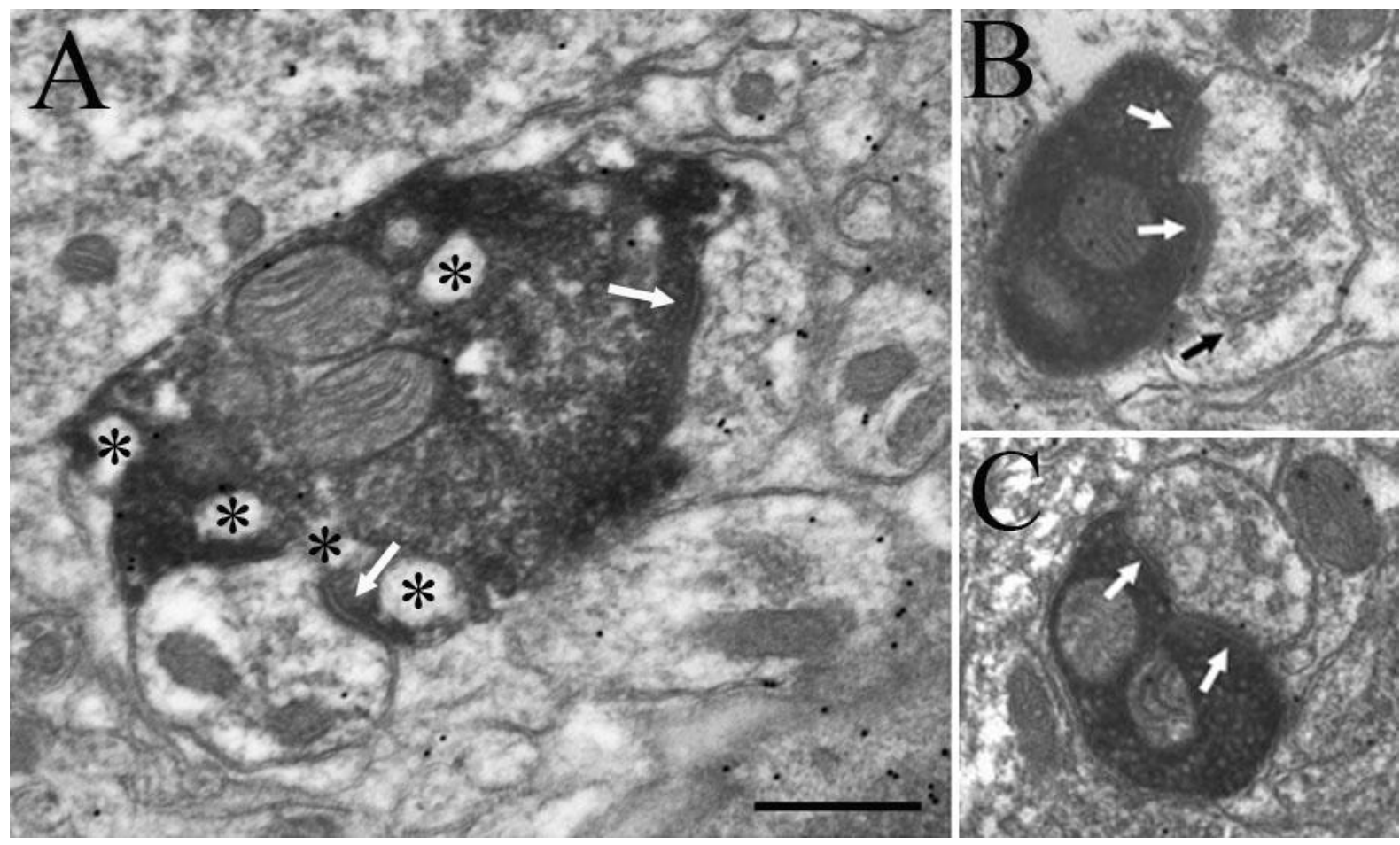
Figure 2.7 Mean size of geniculocortical terminals (GC) in layer IV sublayers of V1. The columns compare mean size of GC terminals labeled either by TdTomato or vGLUT2 in sublayers IVa or IVb of V1. Mean size, $\mu^{2}$. GC labeled by TdTomato in IVa, $n=100$; GC labeled by vGLUT2 in IVa, $n=106$; GC labeled by vGLUT2 in IVb, $n=105$ 


\section{Mean Size of Geniculocortical Connections in Layer IV Sublayers of V1}

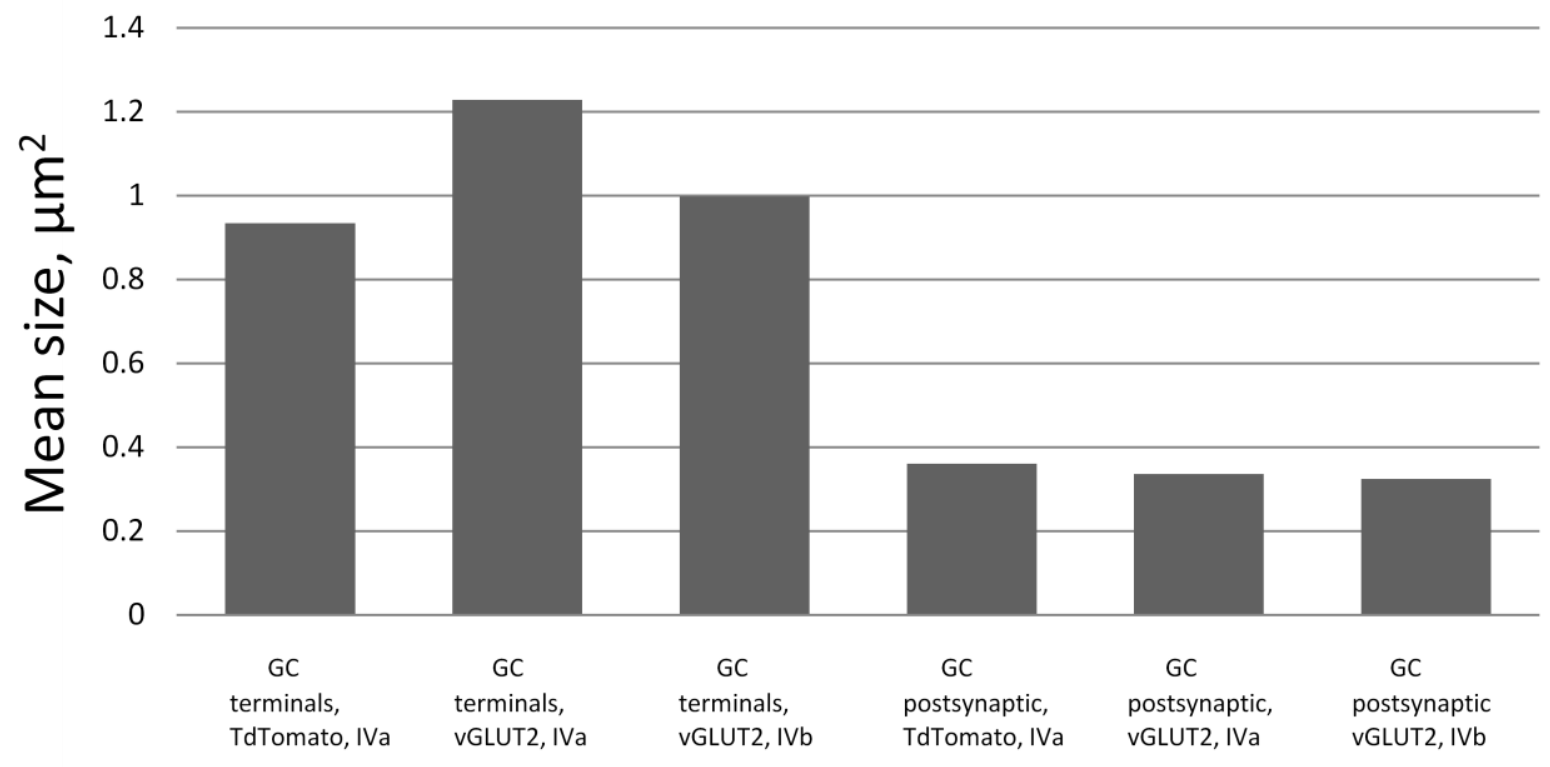


Figure 2.8 GABA-content in synaptic targets of geniculocortical terminals (GC) in layer IV sublayers of V1. Graph shows the content of GABAergic versus non-GABAergic postsynaptic contacts of GC terminals labeled either by TdTomato or vGLUT2 in the sublayers IVa and IVb of V1, percent, \% total. The majority of postsynaptic dendrites contacted by the labeled terminals were non-GABAergic in both sublayers. See results for details of GABA classification. GC labeled by TdTomato in IVa, $n=100$; GC labeled by $v G L U T 2$ in IVa, $n=106$; GC labeled by $v G L U T 2$ in $I V b, n=105$ 


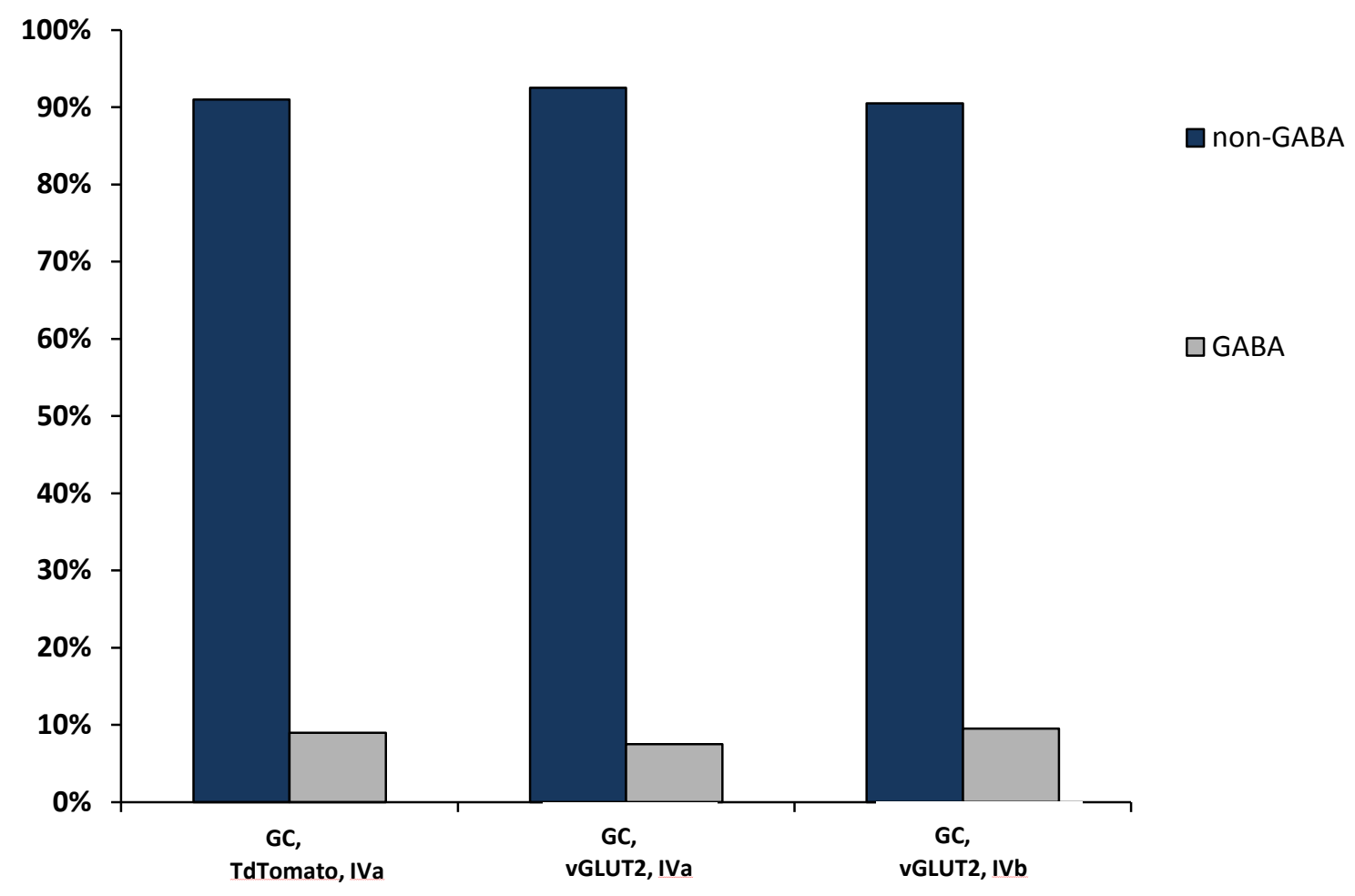


Figure 2.9 Percentage of spine apprati-containing postsynaptic profiles in each type of synaptic contact. Complex types of contacts (perforated or multiple), although less frequently found, were more likely to contain spine apparatus. Total number of each type of contact and percent, \% of spines containing spine apparatus are shown. (A) Spine apparatus content in synapses with simple type of contact, $n=222$. (B) Spine apparatus content in synapses with perforated type of contact, $n=101$. (C) Spine apparatus content in synapses with multiple type of contact, $n=19$ 
A Spine Apparatus Content in Simple Synapses

$\square$ no spine apparatus $\square$ spine apparatus

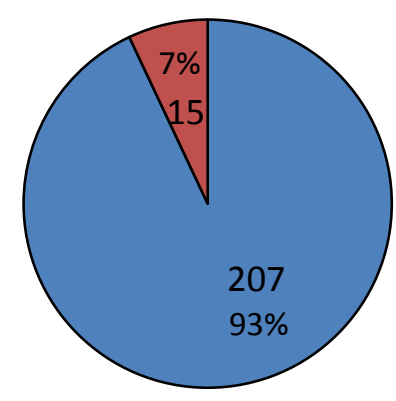

B Spine Apparatus Content in Perforated Synapses

$\square$ no spine appratus $\square$ spine apparatus

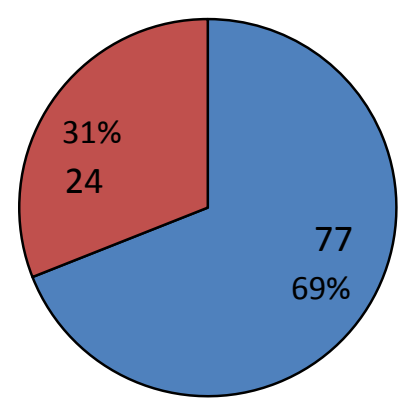

C Spine Apparatus Content in Multiple Synapses

$\square$ no spine apparatus $\square$ spine appratus

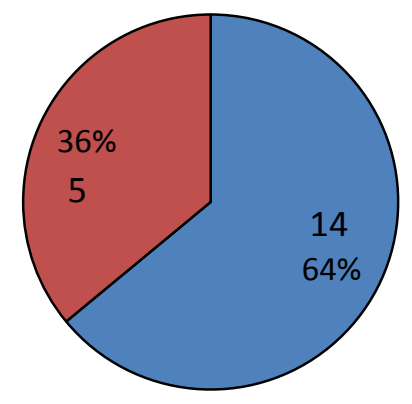


Figure 2.10 Comparison of pre- and postsynaptic sizes of geniculocortical (GC) versus pulvinocortial (PC) terminals. Open yellow squares represent GC terminals and filled black triangles represent PC terminals. The graph demonstrates the distribution of preand postsynaptic sizes of GC terminals in V1 and PC terminals in temporal cortex. The PC terminals are primarily clustered in the lower left portion of the graph, implying that the pre- and postsynaptic profiles have a small area, whereas GC terminals are less clustered indicative of their larger size, $\mu m^{2}$. GC terminals, $n=311$; PC terminals, $n$ $=444$ 


\section{Comparison of Pre- and Post-synaptic sizes in GC and PC}

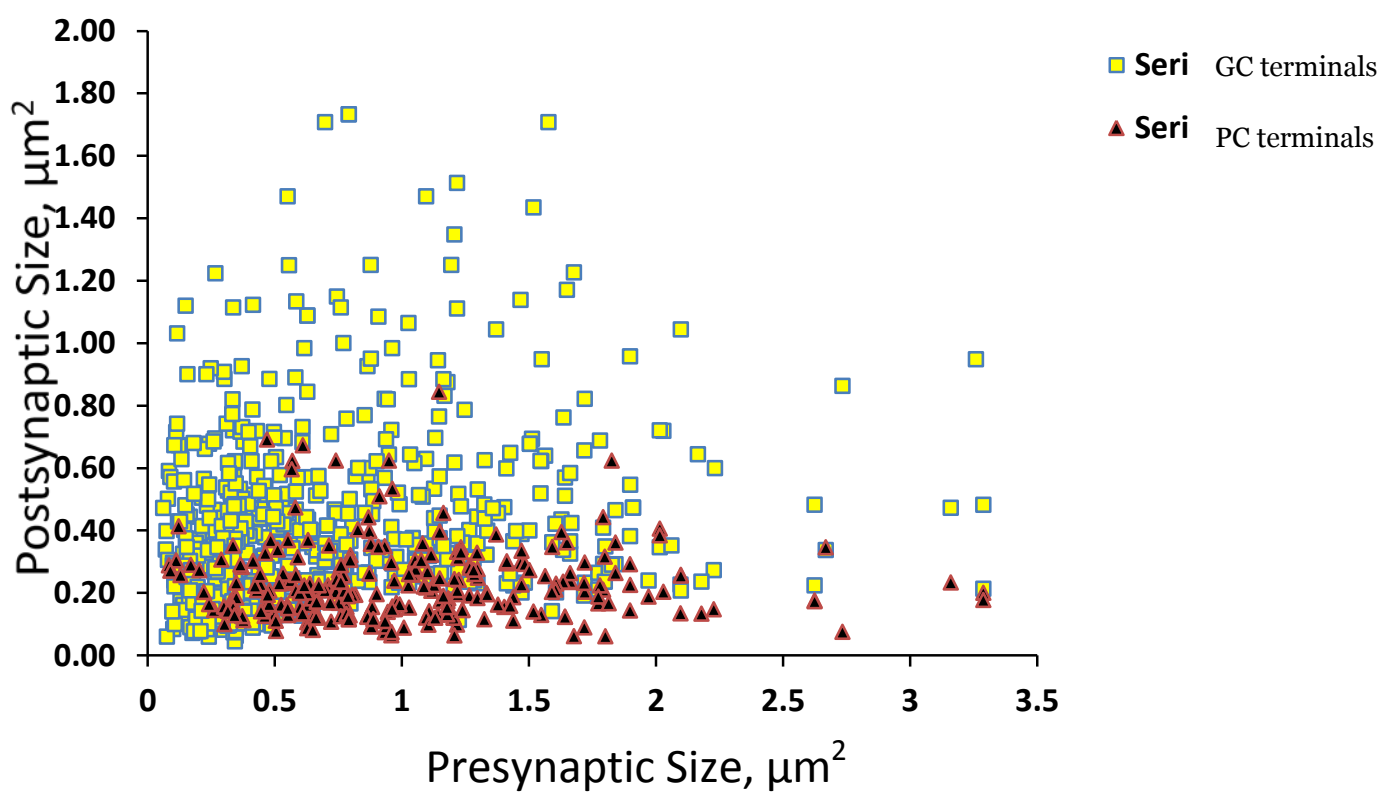




\section{CHAPTER III}

\section{ALTERATION OF SYNAPTOPODIN AND ZINC TRANSPORTER TYPE 3 PROTEIN LEVELS AFTER ZINC PRECIPITATION IN WILD TYPE AND TRANSGENIC MODEL OF ALZHEIMER'S MICE CONTAINING SWEDISH, DUTCH AND IOWA MUTATIONS}

\section{Introduction}

Epidemiological studies indicate that people with increased cognitive and physical activity have a reduced chance of developing Alzheimer's disease (AD) (Wilson et al., 2002; Abbot et al., 2004; Larson et al., 2006; Friedland et. al, 2004; Helzner et al., 2007; Valenzuela et al., 2012). These investigations suggest that neuronal activity may be protective against Alzheimer's disease progression and studies of transgenic mouse models of $\mathrm{AD}$ support this hypothesis. When such transgenic mice are placed into enriched environments, they show significantly lower cognitive decline when compared to controls (Arendash et al, 2004, Arendash et al., 2007; Costa et al., 2007). These effects were especially pronounced when the enriched environment was introduced before the onset of amyloidogenesis (Verret et al., 2013). When enriched housing was introduced at later stages of amyloidogenesis in some severely affected strains no working memory improvement was detected (Wirths et al, 2012). Several studies also showed that an enriched environment reduced beta-amyloid plaque burden in the brains of different transgenic models of AD (Keyvani et al., 2006; Arendash et al., 2007; Sisodia, et al., 2005). However, the mechanisms underlying the influence of different neuronal activity levels in the progression of Alzheimer's pathology still remains unclear.

The beta-amyloid plaques and neurofibrillary tangles, two major morphological hallmarks of $\mathrm{AD}$, affect only telencephalic parts of the brain and they develop in a certain 
sequence. Plaques and tangles first develop in the enthorhinal cortex and subiculum and then the distribution of plaques and tangles slowly progresses to involve whole telencephalon, with their presence noted in neocortex only during the most advanced stages of $\mathrm{AD}$ (Braak, 1993, Braak et al., 2006). The affected neurons in these areas are primarily those that connect to other telencephalon areas; rarely can one identify neurofibrillary tangles or plaques in telencephalic neurons that project to the parts of the brain other than telencephalon (Braak, 1993, Braak et al., 2006).

The very same areas affected by Alzheimer's pathology are distinguished by the presence of glutamatergic synaptic terminals that contain zinc ions and dendritic spines that contain a lamellar arrangement of smooth endoplasmic reticulum known as the spine apparatus. One of the major structural proteins present in the spine apparatus is synaptopodin (Mundel et al., 1997; Deller et al., 2003) (figure 3.1). Both zincergic terminals and dendritic spines are thought to be very plastic; changes in neuronal activity result in corresponding changes in zinc accumulation as well as spine density and spine morphology (Chen et al., 2000; Brown and Dyck 2005; Segal 2005; Yang et al 2008; Corson et al., 2009). Changes in spine morphology and density are postulated to be structural correlates of plasticity involved in higher cognitive functions such as learning and memory (Matus, 2005; Hyman et al., 2007).

A decrease in spine density is thought to be one of the earliest structural changes affecting brains in $\mathrm{AD}$ pathology. The exact causes of the spine loss are not known, but an understanding of activity-dependent mechanisms underlying spine retraction is important for the development of potential treatment options. The co-localization of zincergic terminals, spines with spine apparati and beta-amyloid plaques combined with the evidence of zinc accumulation in the brain areas deprived of the neuronal activity (Brown and Dyck, 2002; Land and Shamalla-Hanna, 2002; Dyck et al., 2003) led to the hypothesis of zinc involvement in restructuring of spine morphology. The evidence of 
zinc along with copper directly participating in beta-amyloid aggregation (Bush et al., 1994; Ha et al., 2007; Bush, Tanzi, 2008; Palumaa, 2009) further supports this hypothesis of zinc homeostasis as an important link in $\mathrm{AD}$ pathophysiology.

\section{Zinc in the brain}

As reviewed by Sensi et al., 2009, 2012, zinc, in its free ionic form, is present within synaptic vesicles at glutamatergic nerve terminals and is synaptically released during neuronal activity. One of the major roles of zinc in the brain seems to lie in its participation in synaptic plasticity through the interaction with $\mathrm{N}$-methyl-D-aspartate (NMDA) glutamate receptors (Paoletti et al., 1997; Rachline, 2005). Co-release of zinc and glutamate in the mossy fibers of hippocampus (the area most abundantly filled with zinc), was shown by Qian and Noebels, 2005. Zinc in the hippocampus was shown to be very important for long-term potentiation processes through targeted interactions with numerous proteins, including ZnR (GPR39) (Besser et al., 2009), TrkB (Li Y, 2001; Huang et al., 2008; Nagappan et al, 208), NMDAR2b (Paoletti et al., 2009), and p75 (NTR) (Lee et al., 2008; Adlard et al., 2010). Thus, modulation of neuronal excitability and participation in synaptic plasticity underlying formation of memory and learning appear to be one of the major roles of zinc in the brain function.

Despite zinc's crucial role in the brain, high levels of it can be neurotoxic. Alterations of zinc homeostasis have been shown to be involved in neuronal injury resulting from epilepsy, ischemia, and brain trauma as well as neurodegenerative diseases such as Alzheimer's disease (Capasso et al., 2005, Frederickson et al., 2005, Mocchegiani et al., 2006; Frazzini 2006; Galasso and Dyck, 2007; Nakashima and Dyck, 2008).

\section{Zinc Transporters}

There are several types of zinc transporters in the brain as reviewed by Nakashima and Dyck, 2008. Zrt-Irt-like proteins (ZIP) move zinc into the cytoplasm 
from the extracellular space and organelles (Gaither and Eide, 2001). To transport zinc out of the cytoplasm, mammals use 10 types of zinc transporter proteins known to date (Cousins et al., 2006). ZnT1 transports zinc out of the cytoplasm, ZnT2 and 4 participate in zinc transport into the endosomes and lysosomes and $\mathrm{ZnT}-5,6,7$ are found on the Golgi apparatus. The roles of ZnT-8, 9 and 10 are not well studied yet. All of the above transporters are ubiquitous and found not only in the brain but also in other tissues. However the zinc transporter type $3\left(\mathrm{ZnT}_{3}\right)$ is exclusively found in the brain and testis and it is responsible for the movement of zinc from the cytoplasm into synaptic vesicles.

The distribution of $\mathrm{ZnT}_{3}$ expression is correlated with the distribution of vesicular zinc in the brain (Palmitter et al., 1996; Lee et al. 2011). Because ZnT3 is the only known protein that regulates vesicular zinc, it was proposed to be an important for cognitive function (Adlard et al., 2010). In experiments with transgenic ZnT3-knockout mice, it was shown that these mice lacked normal vesicular zinc content (Cole et al., 1999). Moreover, these mice exhibited cognitive deficits similar to those found in Alzheimer's disease but only starting at the age of 6 months. Other authors reported deficiency in social and object recognition memory in 3-4 month old ZnT3-knockout mice (Shumyatsky et al., 2011). Adlard et al. (2010) showed that the level of ZnT3 steadily decreases from about two months of age in wild type mice, after 48 years in humans, and large decreases are detected in transgenic mouse models of AD.

\section{Zinc and Alzheimer's disease}

Because zinc facilitates the precipitation of beta-amyloid (A $\beta)$, its role in the pathogenesis of $\mathrm{AD}$ has been investigated intensively. The soluble $\mathrm{A} \beta$ monomers released by neuronal activity from synaptic terminals are constantly degraded by matrix metalloproteinases, but whenever these monomers bind zinc in the vicinity of a terminal, they form oligomers resistant to degradation (Bush et al., 1994; Garai et al., 2006; Sensi et al., 2009). Even zinc deficiency ultimately causes zinc retention by the brain and has 
been shown to increase amyloid plaque burden in transgenic mice (Takeda et al., 2001; Stoltenberg et al., 2007). Extracellular amyloid plaques have also been shown to be enriched in zinc (Lovell et al., 1998; Lee et al., 1999; Miller et al., 2006; Adlard et al., 2008). Double-transgenic mice overexpressing $\mathrm{A} \beta$ and knocked-out by $\mathrm{ZnT} 3$, abolish interstitial (Lee et al., 2002) and vessel-wall (Friedlich et al., 2004) amyloid pathology. In addition to these potential roles in plaque formation, zinc plays an important physiological role in cognitive processes (as described above). Thus, the rapid trapping of Zn2+ by amyloid-beta plaques could affect the potentiation of glutamatergic synaptic connections and contribute to cognitive deterioration (Sensi et al., 2011).

Previous attempts to manipulate zinc levels lead to discovery of the drug with the name PBT2 that is a derivative of the antifungal medication clioquinol. PBT2 is able to precipitate zinc and copper ions in the brain and reduced amyloid plaque burden in several types of $\mathrm{AD}$ transgenic mice (Adlard et al., 2008). Currently this drug is in phase II clinical trials and has shown to improve cognition in Alzheimer's disease patients (Lannfelt et al., 2008; Faux et al., 2010). Further investigations of the mechanism of this drug showed that in the brains of Alzheimer's disease transgenic mice treated with PBT2 there was a significant increase in dendritic spine density, whereas treatment of control mice with PBT2 did not affect spine density (Adlard et al., 2011). These results lead to the possible zinc effect on spine stability in the brain that was further supported by the work of other investigators, who looked at the changes in dendritic spines in Alzheimer's pathology.

\section{Amyloid precursor protein and spine motility}

The early stages of memory loss in $\mathrm{AD}$ are thought to be related to the loss of dendritic spines and their presynaptic connections. There is a significant decrease in spine density in the cortex and hippocampus of human $\mathrm{AD}$ brains and brains of several transgenic mouse models of AD (Catala et al., 1988; Davies et al., 1987; de Ruiter et al., 
1987; Reddy, 2005; Spires-Jones et al., 2007). The events leading to this loss are unclear, but amyloid precursor protein (APP) is suggested to be a key protein involved in the modulation of dendritic spine density.

APP is a transmembrane protein with a large extracellular domain that is thought to interact with adhesive junctions between neurons and/or the extracellular matrix that surrounds neurons. Cleavage of APP with certain enzymes results in the formation of beta-amyloid which is the key component of amyloid plaques. Forms of familial AD with early onset are mostly associated with mutations in genes involved in the processing of APP. Enzymatic cleavage of APP leads to reduced spine stability and the removal of endoplasmic reticulum (spine apparatus) from spines (Storey et al, 1996; Spires-Jones et al., 2007; Ng and Toresson, 2008). It has been shown that direct application of beta amyloid to hippocampal slice cultures is able to transform mushroom-shaped spines to stubby spines, and decrease spine density (Tackenberg and Brandt, 2009; Wei et al., 2010). APP is cleaved by a number of different enzymes, but chief among these are the cell surface metalloproteinases, which are catalyzed by zinc (Adlard and Bush, 2006). Also, as mentioned earlier, the distribution of zincergic terminals is mirrored by the distribution of dendritic spines that contain spine apparati uniquely identified by their association with the actin-binding protein synaptopodin (Mundel et al, 1997). Although neurons with dendritic spines are found in most areas of the brain, only spines within the telencephalon contain spine apparati (Deller et al., 2003; Vlachos et al., 2009).

Possibly, zinc release from less active terminals triggers the cleavage of APP and, as a result, dissolution of the spine apparatus, and transformation of mushroom-shaped spines to smaller, more mobile spines. This process could be beneficial in the normal brain for mediating the plasticity necessary for the formation of new synapses underlying learning and memory. In $\mathrm{AD}$, however, either spine retraction is increased, or spine formation is decreased, so that there is an overall loss of spines and synaptic 
connections. To investigate this interaction we looked at the ways to manipulate zinc levels in the brain and trace the effect of this on dendritic spines and zincergic terminals by measuring synaptopodin levels as well as changes in $\mathrm{ZnT}_{3}$ levels. We decreased zinc levels by precipitating it with sodium selenite. This manipulation results in the formation of nanocrystals of zinc selenite and thereby, decreases the active free ion availability of zinc (Danscher and Stoltenberg, 2006; Doering et al., 2010).

\section{Materials and methods}

A total of 54 mice were used for these experiments. Of these, 27 mice were wild type $\mathrm{C}_{57} \mathrm{BL} / 6$ and 27 mice were the transgenic model of Alzheimer's disease: C57BL/6Tg(Thy1-APPSwDutIowa)BWevn/Mmjax (TgSwDI; this model expresses neuronally derived human amyloid $\beta$-precursor protein, APP gene, 770 isoform, containing the Swedish K670N/M671L, Dutch E693Q and Iowa D694N mutations, under the control of the mouse thymus cell antigen 1, theta, Thy1, promoter). Each group of WT or TgSwDI mice were further subdivided into 3 groups of 9 mice each that were used at 1,4 and 6 months of age (for the examples of pathology at different ages refer to the results in chapter IV).

For each age and strain group, 3 mice were anesthetized with Avertin (0.01 ml/g; stock made as a mixture of $5 \mathrm{gm}$ of tribromoethanol with $5 \mathrm{ml}$ of tert-amyl alcohol; working solution is $\mathbf{2 . 5} \%$ of stock), and as soon they did not show any reflexive responses they were immediately decapitated to collect tissue for western blots. Another group of 3 mice was anesthetized with avertin and then received an intraperitoneal injection of sodium selenite (0.05\% in distilled water; $0.003 \mathrm{ml} / \mathrm{g}$ ) and were sacrificed after 1 hour for tissue collection. The final 3 mice received an intraperitoneal injection of sodium selenite and were sacrificed after 3 hours. 
After each mouse was decapitated, the brain was rapidly removed and after quick rinse in a 0.1M phosphate buffer, $\mathrm{pH}$ 7.4 (PB) was placed into Alto Coronal Brain Matrix (AL-1175; Mouse 45-75Gram; Acrylic; 1mm) and cut into several $1 \mathrm{~mm}$ thick coronal slices using a razor blade. Selected slices were placed on slides and the parietal cortex and hippocampus from the one side of this coronal section were dissected and immediately immersed in liquid nitrogen. In some cases the cerebellum was also removed as a control. Tissue then was prepared for western blot techniques as described below. All the methods used were approved by the University of Louisville Animal Care and Use Committee and conform to the National Institutes of Health guidelines.

\section{Western blots}

The frozen tissue was prepared for electrophoresis. Each piece of tissue was homogenized in Triton lysis buffer (20 mM of 1M Tris, pH 6.8; $137 \mathrm{mM}$ of $5 \mathrm{M} \mathrm{NaCl} ; 25$ $\mathrm{mM}$ of $0.4 \mathrm{M} \beta$-glycerophosphate, $\mathrm{pH} 7.14 ; 2 \mathrm{mM}$ of $0.2 \mathrm{M}$ Na pyrophosphate; $2 \mathrm{mM}$ of 0.5M EDTA; $1 \mathrm{mM}$ of $0.1 \mathrm{M}$ of Na3VO4; $1 \%$ of $10 \%$ triton X-100; $10 \%$ of $50 \%$ glycerol; $1 \mathrm{X}$ of $200 \mathrm{X}$ protease inhibitor cocktail; $0.5 \mathrm{mM}$ of $1 \mathrm{M}$ dithiothreitol; $1 \mathrm{mM}$ of $100 \mathrm{mM}$ phenylmethanesulfonyl fluoride; and double distilled water to needed volume) and sonicated with ultra cell disruptor Microson (Misonix, Inc.) on ice. The lysates were then centrifuged at a speed of $13000 \mathrm{rpm}$ for 30 minutes and the supernatant was separated from the pellet by pipette. The supernatant was then used for protein estimation and loading onto gels. To estimate the protein concentration into each sample, the series of bovine serum albumin (BSA) was used. For that, each sample was diluted 1:10 with double distilled water and loaded into the 3 wells 96-well plate by $10 \mu \mathrm{l}$ per each sample. The six increasing concentrations of $1 \mathrm{mg} / \mathrm{ml} \mathrm{BSA}$ were also loaded into the slots on the same sample plate. Further, 200 $\mu$ m of the Bio-Rad Protein Assay (Bio-Rad Laboratories, Inc., Hercules, CA) added in each slot. The plate then left for 5 minutes and protein concentration estimated with spectrophotometer SpectramaxPlus (Molecular Devices) 
and software (SoftMax Pro, Molecular Devices). Using Microsoft Excel software, the linear plot was created based on the results from spectrophotometer and concentration of protein for 20 micrograms per sample calculated. Then, $200 \mu \mathrm{l}$ of Bio-Rad Laemmli sample buffer (65.8 mM Tris-HCl, $\mathrm{pH}$ 6.8, 2.1\% SDS, 26.3\% (w/v) glycerol, 0.01\% bromophenol blue) and $50 \mu$ of 2-mercaptoethanol in double distilled water were added to complete the $15 \mu \mathrm{l}$ mixture. This samples then were centrifuged for 5 minutes and placed into the hot plate at $95^{\circ} \mathrm{C}$ for 10 minutes. The ready samples were spun once again for 5 minutes and loaded onto gels.

The premade Bio-Rad gels were used for this experiment (Mini-PROTEAN®) TGX Any kD Stain-Free ${ }^{\mathrm{TM}}$ 10-well precast gels with SDS-PAGE accessories, Bio-Rad). Samples were loaded $15 \mu \mathrm{l}$ per well and Bio-Rad Kaleidoscope ${ }^{\mathrm{TM}}$ protein standards were used for detection of molecular weight on the transfer membrane. Molecular weight for synaptopodin is $100 \mathrm{kDa}$, for $\mathrm{ZnT}_{3}$ it is $48 \mathrm{kDa}$ and molecular weight of GAPDH is 39 kDa. Gels were placed into the Bio-Rad running chamber CRITERION ${ }^{\mathrm{TM}}$ Cell and after electrophoresis in Bio-Rad 10x Tris/Glycine/SDS running buffer at 200V was done, proteins from the gels were transferred onto the membrane using Bio-Rad TransTurbo® Blot $^{\mathrm{TM}}$ transferring device, and, after staining with appropriate antibodies, developed using ChemiDoc ${ }^{\mathrm{TM}}$ MP Imager (Bio-Rad) and bands were assessed using Image Lab ${ }^{\mathrm{TM}}$ software.

The following antibodies were used for western blotting in this experiment. Mouse anti- synaptopodin (1:2000, Synaptic Systems Inc.), rabbit anti-zinc transporter type 3 (anti-ZnT3) (1:200o, Synaptic Systems Inc.), and mouse anti-glyceraldehyde-3phosphate dehydrogenase, clone $6 \mathrm{C}_{5}$ (anti-GAPDH) (1:2000, Millipore) as a housekeeping protein. Following transfer from the gels to the membrane, it was washed in $1 \%$ phosphate buffer with saline and Tween20 (PBS-T) solution three times $(20 \mathrm{ml}$ o.5M PB, $9 \mathrm{~g} \mathrm{NaCl}, 1 \mathrm{ml}$ of Tween20) and then places in $5 \%$ non-fat milk solution in PBS- 
$\mathrm{T}$ for 1 hour for blotting non-specific for out antibodies proteins. After 1 hour, membrane was placed into 1:2000 solution of anti-synaptopodin in milk or 1:2000 anti-ZnT3 solution at $4^{\circ} \mathrm{C}$ overnight. Next day, after washing membrane 3 times in PBS-T, the 1:2000 solution of secondary antibodies in milk were applied. Anti-mouse IgG horseradish peroxidase conjugated, SC-2768 (made in goat, 1:2000; Santa Cruz Biotechnology Inc.) for anti-synaptopodin primary and anti-rabbit IgG horseradish peroxidase conjugated, SC-2054 (made in goat, 1:2000; Santa Cruz Niotechology Inc.) for anti-ZnT3 for 1 hour and after 3 washes in PBS-T, the membrane was ready to be examined in ChemiDoc ${ }^{\mathrm{TM}}$ MP Imager. To reveal bands, two commercially available solutions from Bio-Rad were used (Clarity Western ECL Substrate, Bio-Rad) that were mixed right before use and applied onto the membrane for 30 seconds before placing the membrane into the developing chamber.

For the control of protein content and loading differences, the staining of membrane for housekeeping protein GAPDH was used. The process of staining was similar to the described above.

Using Image Lab software, the density of each band (synaptopodin or ZnT3) was measured and divided by the density of GAPDH in the corresponding well to adjust for the differences in the protein content among wells (GAPDH is supposed to be constantly expressed in every animal). Further, the results were grouped in the Excel file and forwarded for statistical analysis to the KSCIRC biostatistics core.

\section{Data analysis}

To analyze the data, statistical analyses were performed using one-way ANOVA procedures and independent t-tests were used to compare the differences in synaptopodin and $\mathrm{ZnT}_{3}$ levels between the wild type mice and transgenic mice models. Also, to analyze the differences across the animals including all three parameters (age, group and time) 3-way ANOVA was used with subsequent use of two-way ANOVA by age 
and group and post hoc t-test comparisons by age and then group. All statistical analyses were performed using IBM SPSS, version 20 software. The graphs were plotted using Sigma Plot, version 11.

\section{Results}

Figure 3.2 shows the bands that were received after developing the image from the membrane. ZnT3 and synaptopodin were detected in the cortex and hippocampus, but not the cerebellum, confirming previous immunocytochemical studies (Paoletti et al., 2009). After the appropriate analysis methods were employed (described above) the following results were obtained (Figure 3.3, 3.4 and 3.5).

\section{Alterations of synaptopodin after zinc precipitation in one month old WT and TgSwDI mice}

We initially investigated whether zinc precipitation affected synaptopodin levels in 1-month old mice when the plastic and metabolic processes in the brain are, presumably, at the highest levels and the Alzheimer-like pathology had not yet developed in the TgSwDI mice. As seen in the figure 3.3, without sodium selenite injection, the levels of synaptopodin were higher in the control animals (WT, black column, o time point) than in the transgenic mice (transgenic, white column, o time point) but this difference did not reach statistical significance for either cortex or hippocampus. After zinc was precipitated with sodium selenite, the level of synaptopodin was measured after 1-hour and 3-hour intervals in WT and TgSwDI mice. However, there was no statistically significant difference in the levels of synaptopodin in the cortex or hippocampus of any of these groups of animals. It is worth noting that in all groups synaptopodin levels were consistently higher in WT versus TgSwDI animals but, again, this difference did not reach statistical significance. 


\section{Alterations of synaptopodin after zinc precipitation in four month old WT and TgSwDI mice}

We next conducted our experiment in more mature, 4 month old mice. According to our data (reported in the next chapter) and that reported by Nostrand et al., 2004, with our transgenic mouse model, the pathological signs of Alzheimer's disease can be detected in 4-month old TgSwDI mice (figure 4.3 in chapter IV). Thus, we considered this age to be the most appropriate time point for investigating whether the disease progression changes zinc metabolism and whether this has an effect on synaptopodin levels. As seen in the figure 3.4, similar to our findings for 1 month old tissue, the level of synaptopodin in 4 month old WT brains was generally higher than in 4 month old TgSwDI brains. However, this difference was not statistically significant. There also were no statistically significant differences in the levels of synaptopodin after sodium selenite treatments in 4 month old animals.

\section{Alterations of synaptopodin after zinc precipitation in six month old WT and TgSwDI mice}

By 6 months of age, the brains of our transgenic mice displayed distinct areas of beta-amyloid deposits, predominantly in the hippocampus (figure 4.3 in chapter IV). As illustrated in Figure 3.5, we found that, as in 1 and 4 month old mice, the cortex of 6 month old WT mice had higher levels of synaptopodin when compared to the cortex of 6 month old TgSwDI mice. This difference reached significance at 1 hour post sodium selenite treatment ( $\mathrm{p}$-value <.o1). However, the opposite result was found in the hippocampus. For this structure, at all three time points, TgSwDI mice showed higher synaptopodin levels when compared to WT mice and this difference was statistically significant for the 1-hour and 3-hour post sodium selenite time points (both p-value $<$ .01). Moreover, in TgSwDI mice, there was a significant increase in synaptopodin levels 1 
hour after the injection of sodium selenite when compared to the levels of synaptopodin in mice without sodium selenite injection (time point $\mathrm{o}$; $\mathrm{p}$-value <.01).

Comparison of synaptopodin alterations after zinc precipitation in WT and TgSwDI at different ages

After the statistical analysis was completed for each age group separately, we next examined whether there were differences in synaptopodin levels across the age groups. We used a 3-way ANOVA and post hoc t-test. The results of this analysis indicate that synaptopodin levels in both the cortex and hippocampus were higher in 4 month old WT mice than in 1- or 6-month old WT mice (both $\mathrm{p}<.01$ ) at all time point related to sodium selenite injection. In TgSwDI mice, synaptopodin levels in the cortex were higher in 4month old animals when compared to 1- and 6-month old animals at the o- and 3-hour time points (both $\mathrm{p}<.01$ ) and synaptopodin levels in the hippocampus were higher in 4 month old animals compared to 1 month old animals at the $0-(\mathrm{p}<.01)$ and 3 -hour $(p<.01)$ time points.

\section{Alterations of $\mathrm{ZnT}_{3}$ after zinc precipitation in one month old WT and TgSwDI mice}

$\mathrm{ZnT} 3$ is essential for the accumulation of zinc within synaptic vesicles (Palmiter et al., 1996; Skangiel-Kramska et al., 2005). Therefore we assessed potential changes in the levels of this transporter related to zinc precipitation or differences in WT versus $\mathrm{TgSwDI}$ animals. As illustrated in figure 3.3 there were no significant differences in $\mathrm{ZnT} 3$ levels in the cortex or hippocampus of one month old WT animals compared to TgSwDI animals, even though the absolute $\mathrm{ZnT}_{3}$ levels were consistently higher in the TgSwDI animals. The precipitation of zinc with sodium selenite failed to alter $\mathrm{ZnT} 3$ levels in any statistically significant manner in one month old WT or TgSwDI animals.

\section{Alterations of $\mathrm{ZnT}_{3}$ after zinc precipitation in four month old WT and TgSwDI mice}


When $\mathrm{ZnT}_{3}$ levels were examined in 4 month old mice, there was a significant differences noted between WT and TgSwDI in both the cortex and hippocampus at oand 1-hour time points (figure 3.4). The TgSwDI showed significantly higher $\mathrm{ZnT} 3$ levels at these time points (all $\mathrm{p}<.01$ ). Again, there was no statistically significant change in $\mathrm{ZnT}_{3}$ levels in WT or TgSwDI when compared at different time points after sodium selenite treatment.

\section{Alterations of ZnT3 after zinc precipitation in six month old WT and TgSwDI mice}

As illustrated in figure 3.5, the analysis of tissue collected from 6 month old mice revealed significantly higher levels of $\mathrm{ZnT}_{3}$ in the hippocampus of $\mathrm{TgSwDI}$ animals compared to the hippocampus of WT animals only at 1-hour time point $(\mathrm{p}<.01)$. All other groups were not found to be significantly different. Thus, significant differences in $\mathrm{ZnT} 3$ levels that we observed in 4 month old mice were lost as the animals aged. Sodium selenite treatment did not alter $\mathrm{ZnT}_{3}$ levels significantly in 6 month old animals.

\section{Comparison of $\mathrm{ZnT} 3$ alterations after zinc precipitation in $\mathrm{WT}$ and $\mathrm{TgSwDI}$ at different ages}

Repeating the post hoc t-test comparison to test for differences in $\mathrm{ZnT}_{3}$ levels across age groups, we discovered that there was no difference in $\mathrm{ZnT} 3$ levels in WT mice of different ages, but there was a significantly higher level of $\mathrm{ZnT}_{3}$ in both the cortex and hippocampus of 4 month old TgSwDI mice when compared to either 1 month old or 6 month old TgSwDI mice (for no sodium selenite treatment and for 1 hour post sodium selenite treatment, all $\mathrm{p}<0.01)$. Also, the cortex of 6 month old untreated TgSwDI mice contained higher levels of $\mathrm{ZnT}_{3}$ levels than the cortex of untreated 1 month old TgSwDI mice $(\mathrm{p}<0.01)$. 


\section{Discussion}

Zinc precipitation in the brain is known to improve cognitive functioning in TgSwDI mice and humans with $\mathrm{AD}$, but the exact mechanism by which zinc participates in $\mathrm{AD}$ and cognition is not clear. Our hypothesis was that zinc is not only released from plaques as one of the proposed mechanism of PBT2 action (Adlard et al., 2008), but its precipitation by selenite might lead to the preservation of mature dendritic spines, which are shown to be progressively lost in Alzheimer's disease (Catala et al., 1988; Davies et al., 1987; de Ruiter et al., 1987; Reddy, 2005; Spires-Jones et al., 2007). The preservation of mature dendritic spines could supply the necessary anatomical basis for potentiation of synapses, and perhaps account for the improvement in cognitive behavior previously reported following zinc chelation or precipitation (Adlard et al., 2008; Lannfelt et al., 2009; Faux et al., 2010).

In fact, spine density has been reported to increase after zinc chelation therapy (Adlard et. al, 2011), but the types of spines that are increased have not been evaluated. In the present study, we wanted to test whether zinc precipitation would have an effect on the most mature dendritic spines, those that contain a spine apparatus and the protein synaptopodin. Our hypothesis was that precipitation of zinc would lead to increases in synaptopodin. We also wanted to see how ZnT3 levels would change with zinc precipitation to assess whether free zinc levels regulate levels of this transporter. Lastly, we wanted to determine whether $\mathrm{ZnT} 3$ or synaptopodin levels were altered in our TgSwDI mice relative to WT mice, and whether $\mathrm{ZnT}_{3}$ or synaptopodin levels change with age or the progression of $\mathrm{AD}$ pathology.

\section{Zinc and synaptopodin}

The results of our experiments showed that the precipitation of free zinc into zinc-selenite nanocrystals did not significantly affect synaptopodin levels in most of the animals tested. However, there was a significant increase in synaptopodin levels in the 
hippocampus of 6 month old TgSwDI after 1 hour of zinc precipitation relative to TgSwDI mice without zinc precipitation. These results could be interpreted in several ways. The first and the most straightforward possibility, is that the free zinc pool does not affect the restructuring of dendritic spines directly either in physiological or pathological conditions. The increase in synaptic density demonstrated by Adlard et al (2011) after the zinc chelator use could be explained by mechanisms related to the effect of this chelator on zinc that is bound to amyloid plaques. Decreasing available zinc could lead to the decreased formation of new plaques, or the destabilization of previously formed plaques to form more soluble oligomers (Ritchie et al., 2003; Dukes et al., 2008; Sensi et al., 2009). This could subsequently facilitate physiological spine re-growth, that is otherwise inhibited by beta-amyloid (Spires-Jones, 2007; Koffie et al., 2009). That would explain the increase in spine density following zinc precipitaiton in TgSwDI but not WT mice found by this research group. This might also explain why we saw an increase in synaptopodin after zinc precipitation only in our oldest age group and only in TgSwDI mice.

One reason that our results differ from those of Adlard et al. (2011) could be the fact that synaptopodin is present only in the most mature spines, while the spine density increase reported by Adlard's group was not differentiated by spine maturity. Another possibility is that the 1- and 3-hour time points used in our experiments were not long enough for mature spines to be formed. The profound effect of sodium selenite toxicity on our experimental animals would not allow us to conduct our investigation at time points longer than 3 hours, while Adlard's group used a less toxic chelator therapy for 11 days. Moreover, they used 4-month and 14-month age groups and a different transgenic model (Tg2576), while our experiments were conducted in younger mice of a different strain. The only significant change we detected in our zinc precipitation experiments was in the hippocampus of 6-month old TgSwDI. As presented in the next chapter, we 
began to detect pathological changes only in the hippocampal region of TgSwDI at this age. Therefore, we may have found more significant changes if we had used older TgSwDI mice. Lastly, unfortunately, our sample size was small. We conducted a power analysis to determine whether changes might be detected if we increased our experimental groups. However, this analysis revealed that the necessary increase in animal numbers was not feasible. Therefore at this point, we must conclude that our results to date do not support our theory that free zinc levels impact mature dendritic spine development or preservation.

\section{Zinc and ZnT3}

When we analyzed our data to determine whether the precipitation of zinc affected levels of the zinc transporter type 3 levels ( $\left.\mathrm{ZnT}_{3}\right)$. We hypothesized that the precipitation of zinc might up-regulate $\mathrm{ZnT}_{3}$ in response but we failed to detect any significant changes in the levels of $\mathrm{ZnT}_{3}$ protein. Possibly, the time frame of our experiments was not enough to change protein expression levels. On the other hand, previous experiments suggest dissociation in the levels of synaptic zinc and $\mathrm{ZnT}_{3}$ during the development of the barrel cortices of young mice. Also, neither short-term nor longterm sensory deprivation that caused an increase in zinc levels led to changes in $\mathrm{ZnT} 3$ levels in adult mice (Skangiel-Kramska, 2005). These results suggest that there may be other mechanisms for regulating $\mathrm{ZnT}_{3}$ expression that do not rely on total zinc levels. Further studies concerning this point are needed to evaluate how $\mathrm{ZnT}_{3}$ levels may change in response to synaptic activity and how $\mathrm{ZnT}_{3}$ levels regulate zincergic and/or glutamatergic transmission.

\section{Synaptopodin and ZnT3 in WT versus TgAD mice}

Finally, we examined our data to determine whether synaptopodin or $\mathrm{ZnT}_{3}$ levels were different in TgSwDI mice relative to WT mice, and whether these levels are age dependent. We did not detect any significant differences in the levels of 
synaptopodin in WT versus TgSwDI until 6 months of age. At this point, several interesting results were noted. Despite the fact that we expected to see a decline in synaptopodin levels as the TgSwDI mice aged, it was in our oldest animals that we discovered that hippocampal synaptopodin levels actually were significantly higher in TgSwDI mice than in WT mice. However, these significant differences were only in the groups that were treated with sodium selenite, at both the 1 and 3 hour time points. This suggests that zinc precipitation can affect spines in older TgSwDI mice. Surprisingly, this effect is so strong that the synaptopodin levels in these mice were higher than that found in WT mice. In contrast, we did not see any differences in synaptopodin levels in the cortex of TgSwDI mice and WT mice, potentially because the cortex is not obviously affected by pathology at 6 months of age (figure 4.3 in chapter IV). Moreover, zinc levels are higher in the hippocampus than in any other area of the brain (figure 3.1). Therefore zinc precipitation may have its greatest effect on spines in the hippocampus.

These observations are even more intriguing when considered in the context of the changes in $\mathrm{ZnT}_{3}$ levels detected in animals of different ages. We found that in tissue collected from 4 month old animals the ZnT3 levels were higher in TgSwDI mice compared to WT mice. This difference was very prominent in both the cortex and hippocampus, suggesting that vesicular zinc transport undergoes significant changes at an age which coincides with the appearance of the first plaques in our TgSwDI model (figure 4.3 in chapter IV). By the age of 6 months there were no significant differences in $\mathrm{ZnT}_{3}$ levels in the cortex of TgSwDI and WT mice, and $\mathrm{ZnT}_{3}$ levels were higher in the hippocampus of TgSwDI relative to WT animals at only one time point.

Our data indicate that ZnT3 levels in WT mice were not different in either the cortex or hippocampus collected at 1, 4 and 6 months of age. In contrast, the ZnT3 levels were higher in tissue collected from 4 month TgSwDI mice than in tissue collected at 1 month or 6 months of age. This suggests that normally ZnT3 levels remain relatively 
constant, but in TgSwDI there is a surge in $\mathrm{ZnT}_{3}$ expression at 4 months of age which is followed by decline back towards initial 1 month levels by 6 months of age. Further complicating the interpretation of our data are two previous studies that examined age related changes in zinc and $\mathrm{ZnT}_{3}$ levels, each of which came to a different conclusion. Adlard et al. (2010) found a decline in zinc and $\mathrm{ZnT}_{3}$ levels as animal aged, whereas Lee et al. (2012) stated that zinc and $\mathrm{ZnT}_{3}$ levels increase with age. The major difference between these studies was the age of the animals investigated. Adlard's group compared 2-8 month old mice while Lee et al compared 12-24 month old mice.

The cause and consequences of such fluctuations in $\mathrm{ZnT}_{3}$ levels requires further investigation. One hypothesis is that entrapment of free zinc in the amyloid plaques causes its depletion for physiological purposes (Adlard et al., 2010). This idea fits very well with the surge of $\mathrm{ZnT} 3$ expression during the initial stages of plaque development. This surge could possibly represent an attempt to overcome a developing zinc deficiency at the synaptic cleft (Deshpande et al, 2009). An amplified expression of $\mathrm{ZnT}_{3}$ in $\mathrm{AD}$ might be sustained for a time, but when the increasing numbers of dystrophic neurons are unable to synthesize $\mathrm{ZnT}_{3}$, the level of $\mathrm{ZnT}_{3}$ would then drop. Further studies will be necessary to determine how $\mathrm{ZnT}_{3}$ levels are regulated during the later stages of Alzheimer's pathology. For now, we can conclude that in our TgSwDI model, major changes in dendritic spine maturation and zinc trafficking take place at the crucial age of 4 months, and that the precipitation of zinc does not necessarily cause an immediate change in ZnT3 or synaptopodin levels. 
Figure 3.1 Zinc and synaptopodin staining of rat brain. (A) Parasaggital section of rat brain stained for zinc (brown) from Paoletti et al., 2009. Zinc is distributed only in the telencephalon. ao, anterior olfactory nucleus, am, amygdala, cp, caudate and putamen, h, hippocampus, s, subiculum, IV, neocortex layer 4. (B) Parasaggital section of rat brain stained for synaptopodin (darker stained areas). Synaptopodin distribution closely mirrors distribution of zinc seen in panel $(A)$, scale bar $=100 \mu \mathrm{m}$ 

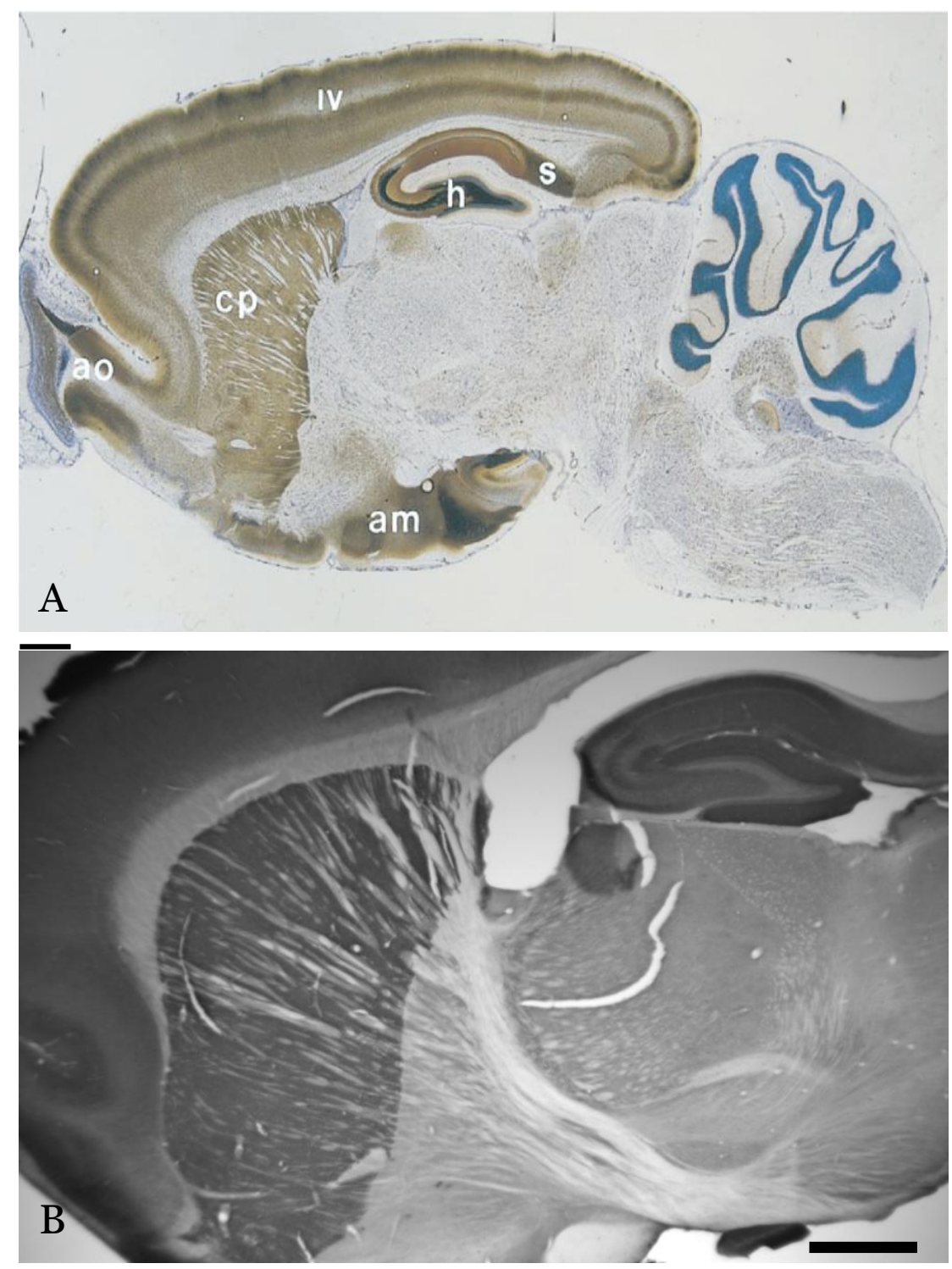
Figure 3.2 The western blot bands from the cortex of 4-month old (A) and 6-month old (B) experimental animals. In the experiment, wild type (WT) and TgSwDI (Tg) mice levels of synaptopodin and $Z n T_{3}$ were compared between the groups and after 1 hour (1h), 3 hours (3h) of intraperitoneal sodium selenite injection. Control animals did not receive sodium selenite (No SS). GAPDH was used as a control housekeeping protein 


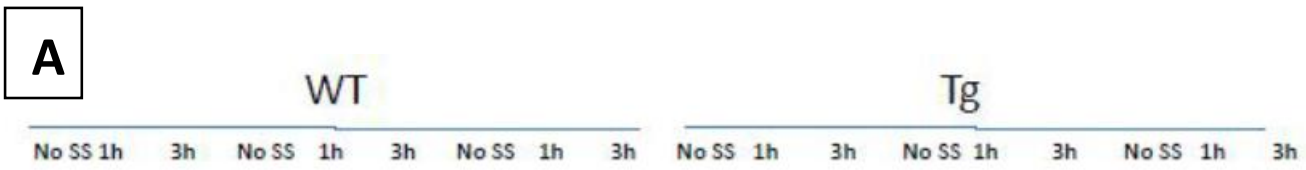

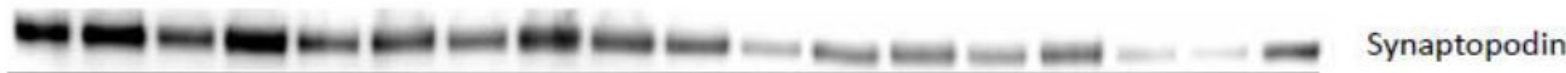

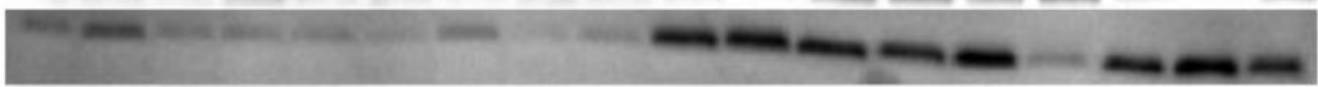

ZnT3

C

GAPDH

4 month old mice

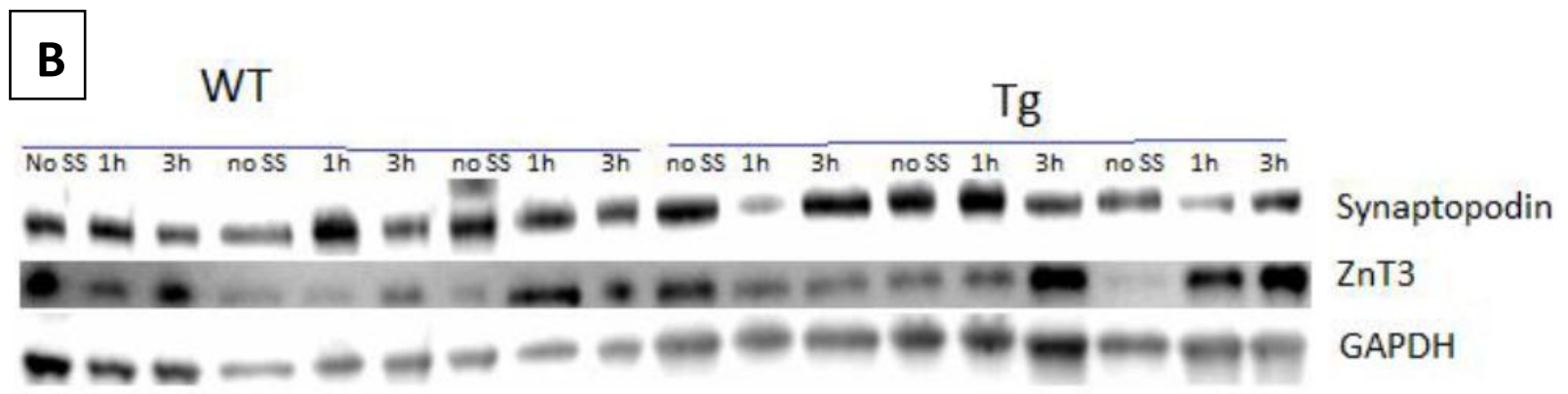

6-month old mice 
Figure 3.3 Synaptopodin and ZnT3 levels before and after sodium selenite treatment in cortex and hippocampus of 1-month old WT and TgSwDI mice. Black columns represent WT mice, each column, $n=3$; white columns represent TgSwDI mice, each column, $n=3$. None of the differences in levels were significant 


\section{Month Old}

CORTEX
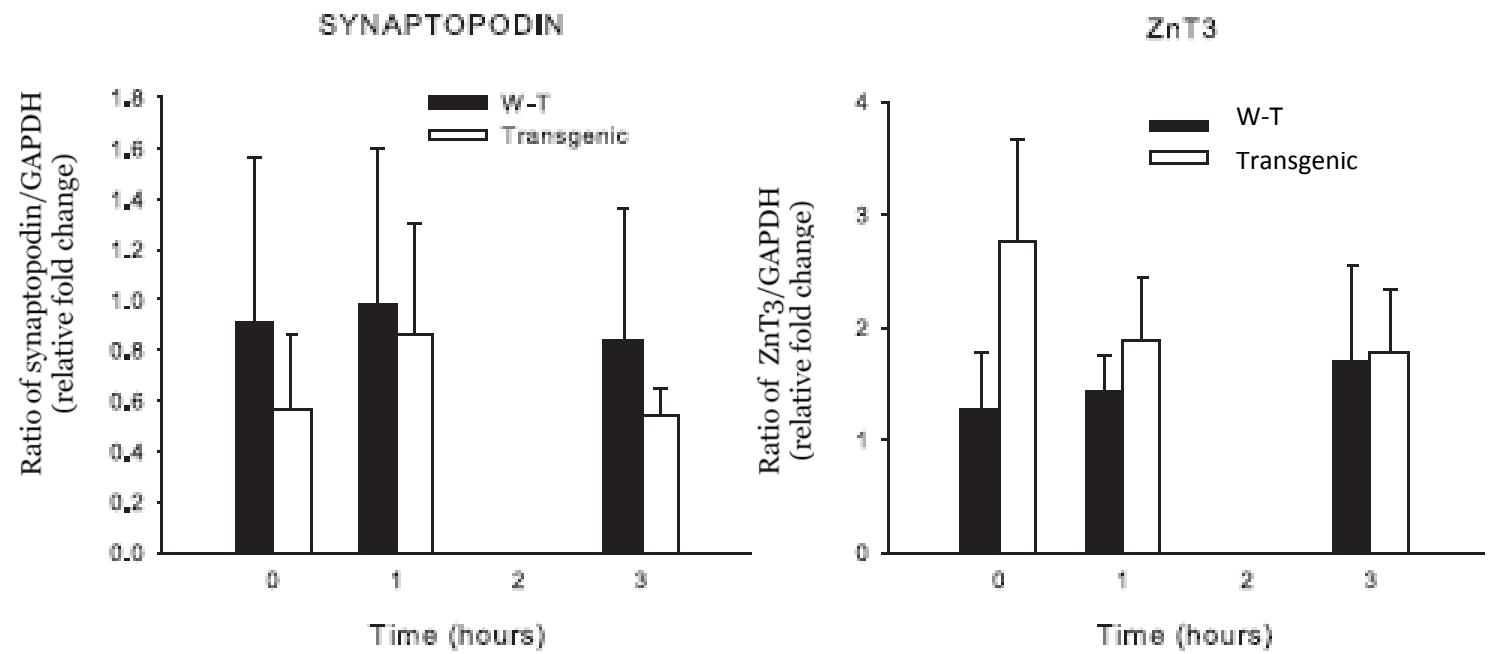

HIPPOCAMPUS
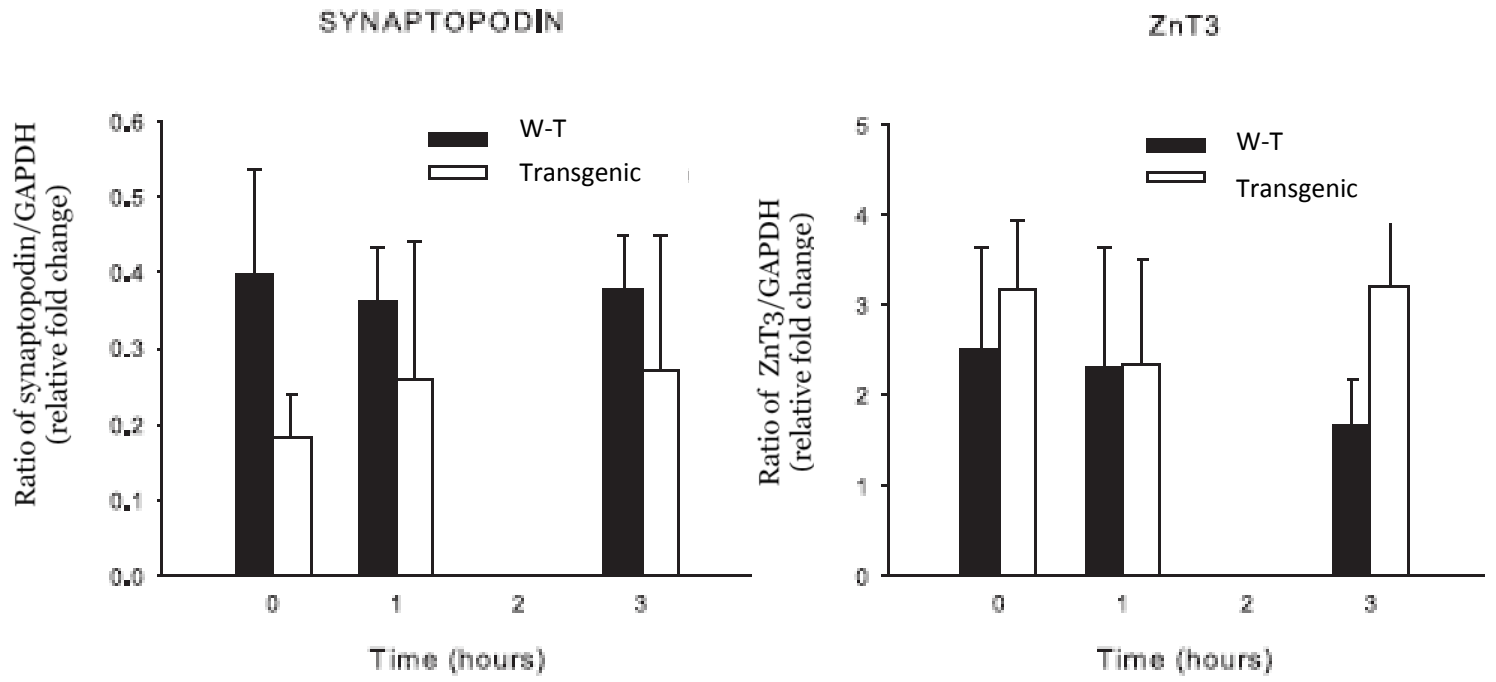
Figure 3.4 Synaptopodin and ZnT3 levels before and after sodium selenite treatment in cortex and hippocampus of 4-month old WT and TgSwDI mice. Black columns represent WT mice, each column, $n=3$; white columns represent TgSwDI mice, each column, $n=3$. Significant differences (asterisks) between WT and TgSwDI were found in the level of ZnT3 in both cortex and hippocampus in controls and at 1-hour time point, $p$-value $<.01$ 
4 mo. old

CORTEX

SYNAPTOPODIN
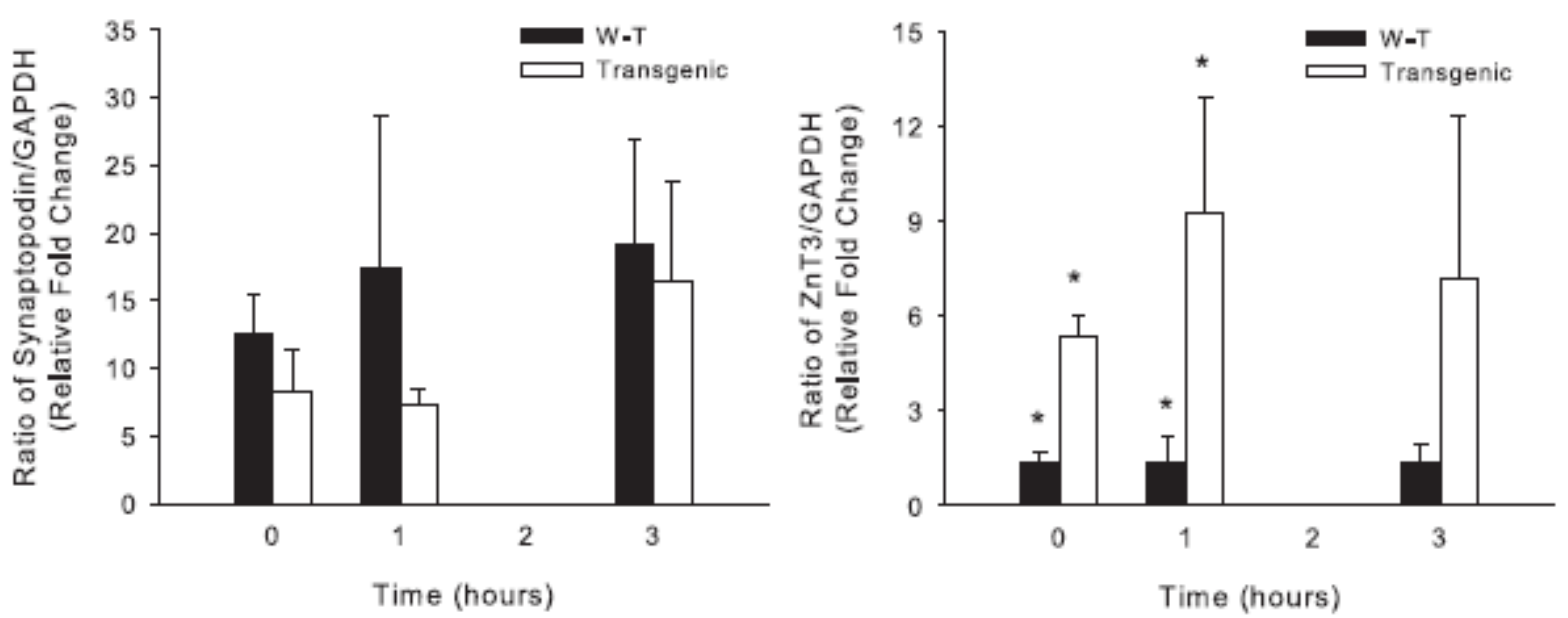

HIPPOCAMPUS

SYNAPTOPODIN

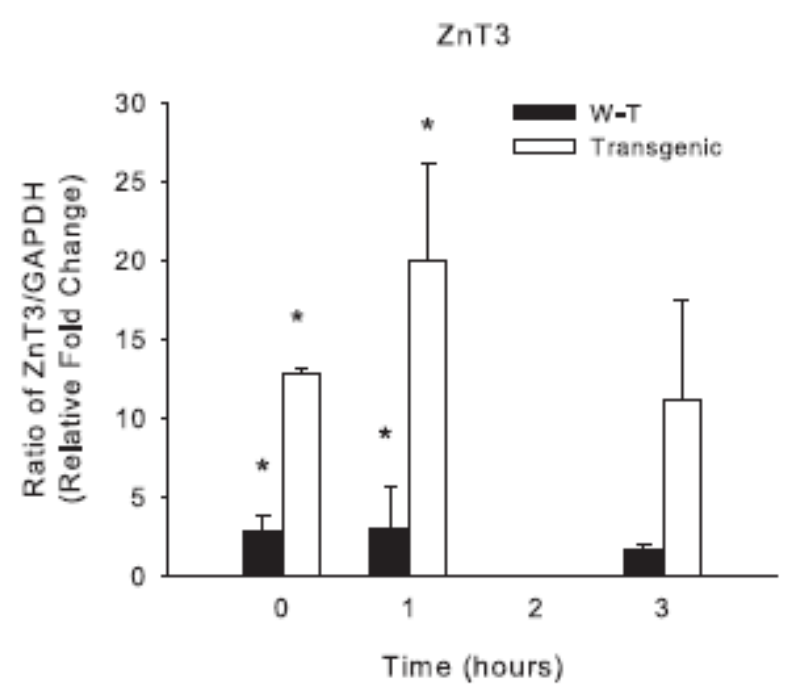


Figure 3.5 Synaptopodin and ZnT3 levels before and after sodium selenite treatment in cortex and hippocampus of 6-month old WT and TgSwDI mice. Black columns represent WT mice, each column, $n=3$; white columns represent TgSwDI mice, each column, $n=3$. Significant differences (asterisks) between WT and TgSwDI were found in the level of synaptopodin in both cortex (at 1-hour) and hippocampus (at 1- and 3hours) and $Z n T_{3}$ in hippocampus at 1-hour time point, all p-values <.o1. Also, significant difference in synaptopodin level was found after 1-hour of sodium selenite treatment (upward arrowheads) when compared to control in hippocampus, p-value $<.01$ 
6 mo. old

CORTEX

SYNAPTOPODIN

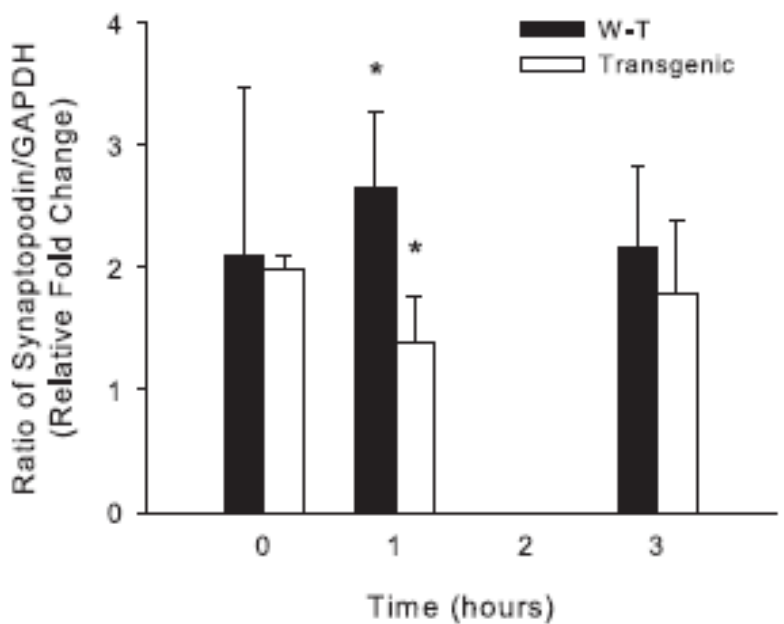

ZnT3

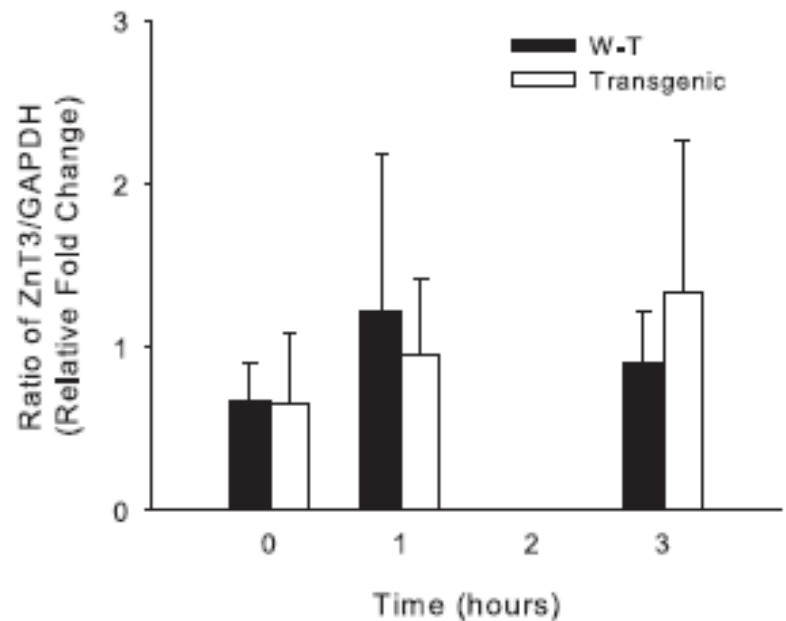

HIPPOCAMPUS

SYNAPTOPODIN
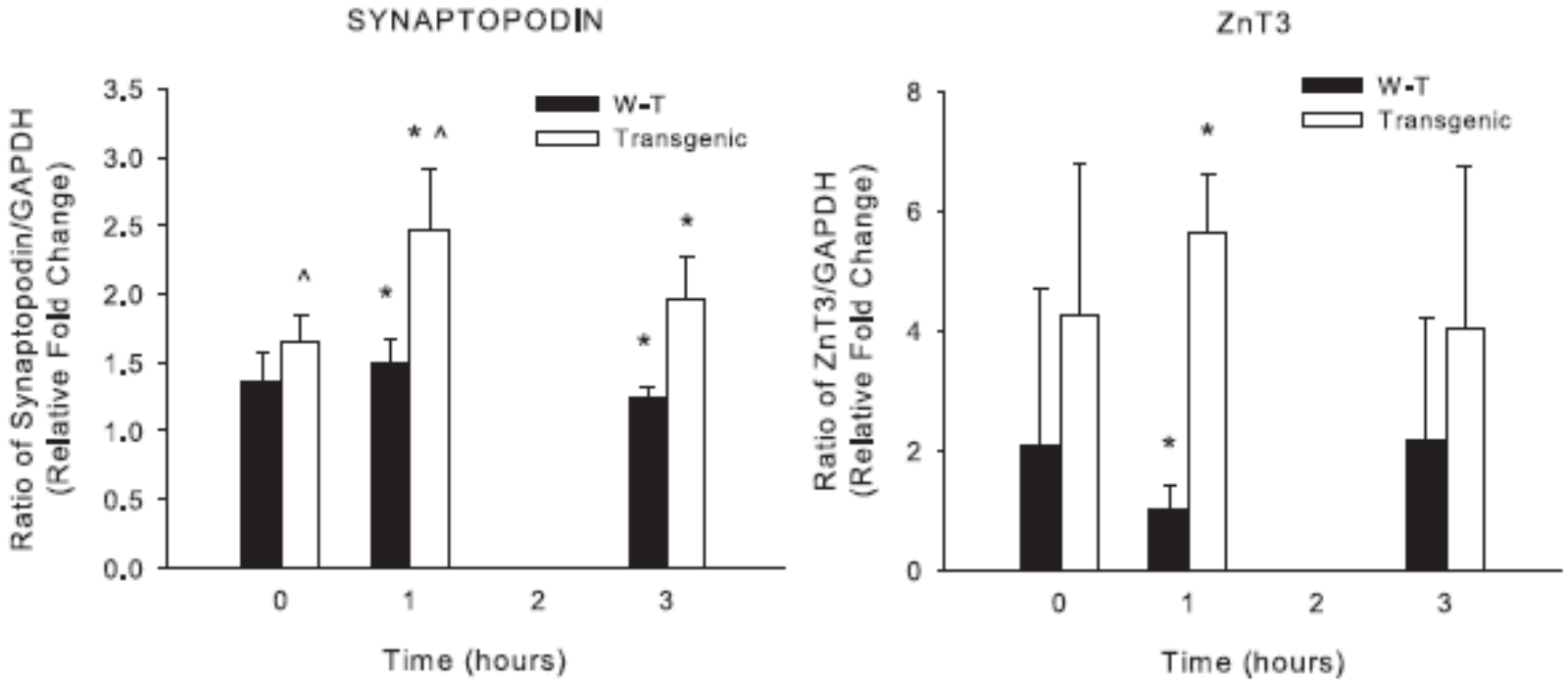


\section{CHAPTER IV}

\section{BETA-AMYLOID PLAQUE FORMATION: HISTOCHEMICAL AND ULTRASTRUCTURAL CHARACTERISTICS IN A TRANSGENIC MOUSE MODEL OF ALZHEIMER'S DISEASE CONTAINING SWEDISH, DUTCH AND IOWA MUTATIONS}

\section{Introduction}

Beta-amyloid $(\mathrm{A} \beta)$, or senile, plaque is a major histopathological hallmark of Alzheimer's disease (AD). Extensive work by previous investigators has revealed the major ultrastructural constituents of amyloid plaques in human tissue (Kidd, 1963, 1964; Terry, 1963; Terry et al., 1964; Wisniewski and Terry, 1973). Extracellular deposits of amorphous material represent the core of the plaque. This core consists of $\beta$-amyloid fibrils, produced from amyloid precursor protein (APP) after sequential proteolytic cleavage by $\beta$ - and $\gamma$-secretases (Kang et al., 1987; Selkoe et al., 2001; Pluta et al., 2013). The beta-pleated sheet structure of this protein confers the ability of it to bind to the dye Congo red, producing birefringence when inspected under polarized light; this is a defining property of the amyloid (Perl, 2010).

When viewed with a light microscope, senile plaques are generally seen as a single morphological unit, but the extensively studied $A \beta$ is not the only constituent of these plaques. Although the term "diffuse plaque" is used to define extracellular agglomerates of $\mathrm{A} \beta$ fibrils with minimal adjacent neuritic dystrophy (Yamaguchi et al., 1989), the most pronounced pathological sign of $\mathrm{AD}$ is characterized by abnormal neurites that surround an amyloid core. These abnormal neurites are composed of the axons or dendrites of dystrophic, dying or dead neurons that contain dense bodies, 
membranous profiles, and packets of paired helical filaments (Perl, 2010). There is a significant loss of synaptic connections within these abnormal areas, and this is thought to be the major correlate of cognitive decline in affected AD patients (DeKosky and Scheff, 1990; Terry et al., 1991; DeKosky et al., 1996; Scheff and Price, 2003; Thind and Sabbagh, 2007; Knafo et al., 2012). The plaques that exhibit a corona of dystrophic neurites surrounding a central core center filled with $\mathrm{A} \beta$ are generally described as "coreonly plaques" or "neuritic plaques". The term "burnt-out plaque" also occurs in the literature; this refers to plaques that present a dense core of $\mathrm{A} \beta$ without any adjacent cells (Thal et al., 2000).

Reactive astrocytes and microglia are other important components of the neuritic plaques (Frackowiak et al., 1992; Wegiel et al., 2000; Stalder et al., 1999, 2001; Nagele et al., 2004). Clinical work and studies of $\mathrm{AD}$ animal models suggest that microglial activation precedes the formation of plaques or tangles (Griffin et al., 1989; Heneka et al., 2005; Solito and Sastre, 2012). However, after several decades of investigation, the role of microglia in the progression of $\mathrm{AD}$ is still debated. Microglial involvement in $\mathrm{AD}$ pathology could be beneficial, via clearance of $A \beta$, phagocytosis of damaged cells, and release of neuroprotective anti-inflammatory factors. On the other hand, microglial involvement in $\mathrm{AD}$ could be harmful due to excessive generation of reactive oxygen species (Solito and Sastre, 2012; Sierra et al, 2013).

The distribution of senile plaques in the brain progresses in a stereotyped manner. Braak and Braak, 1991 first described the stages of AD pathology and this was modified in later work (Thal et al., 2006). According to Braak and Braak, the initial plaque distribution begins in the phylogenetically oldest part of the brain, the allocortex, which includes the olfactory bulb, entorhinal region, presubicular region, and hippocampal formation. As the disease progresses, plaques and tangles are found in the mesocortex, which includes the orbitofrontal region, cingluate gyrus and the 
parahippocampal gyrus. In the final stages of the disease, plaques and tangles are distributed in the neocortex.

The stage of Braak and Braak primarily considered the formation of neuritic plaques. Later investigations of plaque progression have additionally taken into account the formation of diffuse plaques. If diffuse plaques are considered, these are found prior to neuritic plaques and are first seen in the neocortex. These are generally first seen in the temporal cortex and from there progress to the subiculum/CA1 and enthorhinal regions and then other allocortical regions (Thal 2002; 2006). When compared to neuritic plaques, diffuse plaques are thought to be the less detrimental to cognitive function because they are seen even in the normally aging brains of unaffected individuals (Price et al., 1991; Arriagada et al., 1992). However, it is still unclear whether diffuse plaques represent a different pathological process or if diffuse plaques represent and early stage in the development of neuritic plaques.

To begin to shed light on this issue, we examined the development of plaques in a transgenic mouse model of $\mathrm{AD}$. We use the triple mutant $\mathrm{C} 57 \mathrm{BL} / 6-\mathrm{Tg}$ (Thy1APPSwDutIowa) BWevn/J. These transgenic mice express neuronally derived human amyloid beta-precursor protein, APP gene, 770 isoform, containing the Swedish K670N/M671L, Dutch E693Q and Iowa D694N mutations, under the control of the mouse thymus cell antigen 1, theta, Thy1, promoter. The major efforts in creating transgenic mouse models of $\mathrm{AD}$ have been directed to the overexpression of $\mathrm{APP}$, mutated to reflect the known APP mutations that are seen in familial forms of AD that is referred as "human" APP (hAPP). The APP gene contains 19 exons 7, 8 and 15 of which are subjects to alternative splicing. They form different APP isoforms designated by the number of amino acids (Mucke et al., 1995). The longest of these isoforms, APP770, was targeted in our model to introduce three mutations, named after the origin of the families that were first discovered to carry each of those mutations. First, Swedish 
K670N/M671L, with the altered codons 670/671, is a double mutation in the site coding for two amino acids immediately preceding the $\beta$-secretase cleavage site; this induces the increased cleavage of APP by $\beta$-secretase and results in more production of $A \beta 40$ and Aß42 (Selkoe et al., 2001). Second, Dutch E693Q (the number corresponding to APP770 isoform numbering; also known as Dutch $\mathrm{E} 22 \mathrm{Q}$ if considering the $\mathrm{A} \beta$ sequence numbering) is a single point mutation which leads to an increase in aggregation rates of $\mathrm{A} \beta$ by lowering the barrier for $\mathrm{A} \beta$ monomer deposition into a fibril (Levy et al., 1990; Baumketner et al., 2008). Introduction of this mutation also leads to the development of pronounced cerebrovascular $\mathrm{A} \beta$ deposition, or, cerebral amyloid angiopathy, that can be detected in 90\% of AD patients (Vinters, 1987; Davis and Van Nostrand, 1996; Schaeffer et al., 2011). Third, Iowa D694N (or D23N) missense mutation has also shown to increase the $A \beta$ fibril assembly by changing the $\beta$-sheets conformation of $A \beta 40$ fibrils (Van Nostrand et al., 2001; Meredith et al., 2009). This mutation is also associated with severe cerebral amyloid angiopathy, but, unlike the Dutch mutation, the main representation of which was cerebral hemorrhages and dementia, the Iowa D694N is complimented by widespread neurofibrillary tangles, and unusually extensive distribution of $\mathrm{A} \beta 40$ in plaques in $\mathrm{AD}$ brains (Grabowski et al., 2001; Shaeffer et al., 2011). Moreover, since both the Dutch and Iowa mutations lead to the loss of a negative charge in $\mathrm{A} \beta$, the combination of those two result in more robust fibrillogenic and pathogenic properties in cerebrovascular smooth muscle cells and brain parenchyma (Van Nostrand et al., 2001). Lastly, the Thy-1 promoter was used to increase the expression of the mutations of "humanized" APP mRNA levels (Andra et al., 1996).

Interestingly, despite attempts to increase the expression of APP, this mouse model showed decreased expression of APP compared to endogenous levels of APP in wild type mice, suggesting that overexpression of APP is not necessary for developing AD pathology. Furthermore, it was shown that clearance of mutant $A \beta$ into the circulation 
was poorer in this transgenic model than in wild-type mice. Amyloid accumulation in cerebral blood vessels and diminished low-density lipoprotein-1 (LRP-1)-mediated transport across the blood-brain barrier in the cerebral microvasculature is attributed to this A $\beta$ clearance deficit (Deane et al., 2004; Van Nostrand et al., 2004; 2005; 2006). Previous investigators have pointed out that in this $\mathrm{AD}$ mouse model the vascular deposits are fibrillar, but the deposits in the parenchyma primarily exhibit the diffuse type of plaque; this is in accord with the type of pathology seen in human carriers of the Dutch and Iowa mutations (Levy et al., 1990; Grabowski et al., 2001; Van Nostrand et al., 2004). It has also been mentioned that in this mouse model, the classic, neuritic or core amyloid plaques, though seen occasionally in the parenchyma, were a very rare event and did not appreciably increase with the age (Van Nostrand et al., 2004). Most importantly, the transgenic mice that we used for the current study have shown deficits in spatial learning and memory tasks, implying that despite the fact that lack of classic $\mathrm{AD}$ pathology, the memory impairments are still pronounced (Van Nostrand et al., 2007).

We used both light and electron microscopy to examine the distribution and morphology of plaques in transgenic mice of different ages. To determine the extent of microglial involvement we used isolectin B4 (Ib4) which binds to a-D-galactosyl that is found on the cytoplasmic and intracytoplasmic membranes of reactive microglia (Streit and Kreutzberg, 1987; Streit, 1990). We then examined the ultrastructure of neurons and glial cells in the vicinity of the plaques identified by IB4 staining to characterize the extent of neuronal dystrophy. We found that activated microglia were concentrated in areas where microvascular fibrillar amyloid content was the highest, suggesting that microglial activation is strongly related to the vascular pathology (Van Nostrand et al., 2005). We also describe the progressive increase in the distribution of activated microglia as the mice age. 


\section{Materials and Methods}

A total of 17 mice were used for the experiments. Thirteen mice were C57BL/6-Tg (Thy1-APPSwDutIowa) BWevn/J, a transgenic model of Alzheimer's disease (TgSwDI; this model expresses neuronally derived human amyloid $\beta$-precursor protein, APP gene, 770 isoform, containing the Swedish K670N/M671L, Dutch E693Q and Iowa D694N mutations, under the control of the mouse thymus cell antigen 1, theta, Thy1, promoter). Five TgSwDI mice were used for transmitted light microcopy (ages 1, 4, 6, 8 and 14 months). Three TgSwDI mice were used for confocal microscopy (ages 1, 4 and 13 months). Five TgSwDI mice were used for electron microscopy (ages 1, 4, 6, 14 and 20 months). Electron microscopy results were compared to tissue obtained from one 24 month old Tg2576 mouse (containing only one Swedish mutation in APP gene). As a control for both light and confocal microscopy, 3 wild type $\mathrm{C}_{57} \mathrm{BL} / 6$ mice were used (ages 21 days, 4 and 6 months). All the methods used were approved by the University of Louisville Animal Care and Use Committee and conform to the National Institutes of Health guidelines.

All mice were given a lethal dose of Avertin $(0.3 \mathrm{ml} / \mathrm{g})$ by intraperitoneal injection (for Avertin preparation, see materials and methods in chapter III). After this, the mice were transcardially perfused with either a solution of $4 \%$ paraformaldehyde in o.1M phosphate buffer $\mathrm{pH} 7.4$ (PB; for transmitted light and confocal microscopy) or a solution of $2 \%$ paraformaldehyde-2\% glutaraldehyde in PB (for electron microscopy). The mice were then decapitated and the fixed brains were carefully removed from the skulls. The brains were cut into $50 \mu \mathrm{m}$ thick parasagittal sections using a vibratome and the sections were collected and placed in $\mathrm{PB}$. The sections were then processed to reveal the presence of amyloid or the type 3 zinc transporter (ZnT3; using 
immunohistochemistry), or activated microglial cells (using the binding of Griffonia simplicifolia isolectin $\mathrm{B} 4$; IB4), or prepared for electron microscopy as described below.

\section{Amyloid and ZnT3 staining for transmitted light microscopy}

Sections were incubated in a solution of $10 \%$ normal goat serum (NGS) and $0.1 \%$ Triton X-100 in phosphate buffered saline (0.01M PB with $0.9 \% \mathrm{NaCl}$; PBS) for 30 minutes and then placed in antibody solutions overnight at $4^{\circ} \mathrm{C}$. To reveal the presence of amyloid, sections were incubated in 1:100o dilution of the mouse anti-amyloid antibody (clone 4G8, a synthetic polypeptide binding to 17-24 residues of beta-amyloid; Cat. No. SIG-39220, Covance) in 1\%. NGS and PBS. To reveal the presence of ZnT3, sections were incubated in a 1:2000 dilution of rabbit anti-ZnT3 (Cat. No. 197 002, Synaptic Systems Inc.) in 1\% NGS and PBS.

The next day the sections were rinsed in PB (three 10 minute washes) and then transferred to 1:100 dilutions of either biotinylated goat anti-mouse IgG (to reveal amyloid) or goat anti-rabbit IgG (to reveal ZnT3; Vector Laboratories, Burlingame, CA) in $1 \%$ NGS/PBS for 1 hour at room temperature. The sections were then rinsed in PB (three 10 minute washes) and incubated for 1 hour in a solution of avidin and biotinylated horseradish peroxidase (ABC solution, Vectastain, Vector Laboratories, Inc., Burlingame, CA). The sections were then rinsed in PB (three 10 minute washes) and subsequently reacted with nickel-enhanced diaminobenzidine (DAB) for 5 minutes. After thorough rinses in $\mathrm{PB}$, the sections were mounted on slides, dehydrated in the alcohol series, cleared in a solution of citrosolve, and coverslipped for light microscopy examination. A Leica DMLB microscope equipped with a camera Exi Aqua from QImaging was used to capture images of the stained sections.

\section{IB4 staining for transmitted light microscopy}

To reveal the presence of activated microglial cells (Streit and Kreutzberg, 1987; Streit, 1990, Streit et al., 2007), sections were incubated overnight in a 1:1000 dilution of 
biotinylated-IB4 (Cat. No. I21414, Invitrogen) in PBS. The next day the sections were rinsed in $\mathrm{PB}$ (three 10 minute washes) and incubated for 1 hour in $\mathrm{ABC}$ solution. The sections were subsequently rinsed, reacted with $\mathrm{DAB}$, mounted and coverslipped, and images captured as described above.

\section{Amyloid, ZnT3 and IB4 staining for confocal microscopy}

For confocal microscopy, sections were incubated overnight in primary antibodies and IB4 as described above. The sections were then rinsed in PB and incubated for 1 hour in a solution of $1 \%$ NGS and PBS containing a 1:100 dilution of a goat-anti-mouse antibody conjugated to Alexafluor-488 (to reveal amyloid; Cat. No. A11059, Invitrogen), a 1:100 dilution of a goat-anti-rabbit antibody conjugated to Alexafluor-546 (to reveal ZnT3; Cat. No. A110o8) and a 1:100 dilution of strepavidin conjugated to Alexafluor-633 (to reveal IB4; Cat. No. S21375; Invitrogen). The sections were subsequently rinsed in $\mathrm{PB}$, mounted on slides and coverslipped with Prolong® Gold Antifading reagent (Invitrogen). Images of the sections were then captured with an Olympus Fluoview confocal microscope.

\section{Electron microscopy}

To prepare the tissue for electron microscopy, selected sections from TgSwDI animals were postfixed in $2 \%$ osmium tetroxide, dehydrated in ethyl alcohol series and incubated in Durcupan resin overnight in a vacuum chamber. The next day, these sections were flat embedded in Durcupan resin between two sheets of Aclar plastic (Ladd Research, Williston, VT) and cured for 48 hours at $60^{\circ} \mathrm{C}$. Using a light microscope, the embedded sections were examined to selected areas of interest. These selected areas were then mounted on resin blocks and ultrathin sections $(70-80 \mathrm{~nm})$ were cut with an ultramicrotome using a diamond knife. Ultrathin sections were collected on Formvarcoated nickel slot grids. After air drying the sections were stained with a 10\% solution of 
uranyl acetate in methanol for 30 minutes. They were then examined with a Phillips CM10 electron microscope and images were captured using SIA-12C camera.

\section{Results}

\section{Amyloid and IB4 staining co-localize in TgSwDI mice}

As illustrated in Figures 4.1 - 4.3, we found that, in tissue collected from TgSwDI mice, amyloid antibody staining was difficult to detect before 13 months of age. At this age, we found that binding of the IB4 robustly stained all areas that were stained with the amyloid antibody (Figures 4.1 and 4.2). In the cortex, both staining methods revealed areas resembling previous descriptions of amyloid plaques. DAPI staining of the same sections revealed a large number of glial cell nuclei distributed in the vicinity of the plaques (Figure 4.1), suggesting that IB4 staining reveals the presence of activated microglia, as previously reported (Streit and Kreutzberg, 1987; Streit, 1990). In the hippocampus, amyloid and IB4 staining overlapped in the mossy fiber area, a region known to be one of the earliest areas affected by amyloid plaque pathology (Braak and Braak, 1991).

\section{Progression of IB4 staining with age}

We utilized IB4 binding to examine the progression of plaque deposits in tissue collected from TgSwDI mice of different ages. As a control, we stained tissue collected from P21 WT mice (Figure 4.3A) and did not find any signs of staining in any areas of the brain. In tissue collected from 4-month old TgSwDI mice, we detected a small amount of IB4 staining in the subiculum area, immediately ventral to the forceps major of the corpus callosum (Figure 4.3B). In 6 month old TgSwDI tissue, the IB4 staining expanded to include the CA1 and CA2 hippocampal areas along the alveus and oriens layers of the hippocampus, and a few labeled spots were detected in the mossy fibers area (Figure 4.3 C). Also, in tissue from 6 month old mice, labeled aggregates of presumed activated 
microglia were detected in the deep layers of the neocortex dorsal to the hippocampus. In 14-month old TgSwDI tissue, IB4 staining was detected in most of the hippocampus and surrounding areas, from the subiculum through $\mathrm{CA}_{1}, \mathrm{CA} 2$ and $\mathrm{CA}_{3}$ to the densely labeled mossy fibers (Figure 4.3D, E). Thus at 14 months of age, the IB4 staining was mostly distributed within areas of major hippocampal connections, such as the alveus, mossy and Schaeffer fibers. By 14 months, IB4 staining was also observed in the lower layers of the surrounding cortex, with occasional diffuse labeling in the more superficial layers of visual and somatosensory cortex. By 20 months of age, TgSwDI IB4 staining was observed in the hippocampus, many areas of the neocortex, as well as the striatum (Figure 4.4). Examination of IB4 staining at high magnification revealed that the isolectin binding was often concentrated around blood vessels in both the cortex and subiculum as well as in olfactory bulb (figure 4.5)

\section{Ultrastructure of plaques in Tg2576 mice}

We first examined the ultrastructure of plaques in tissue obtained from a 24 month old Tg2576 mouse. This mouse model of Alzheimer's disease is known to produce plaques that display morphological characteristics similar to those observed in human tissue (Terry et al., 1963; Masliah et al., 1996; Dikranian et al., 2012; Hsiao et al., 1998; Irizarry et al., 1997; Takahashi et al., 2002). Figure 4.6 illustrates an example of a mature plaque from the frontal cortex of the 24-month old Tg2576 mouse. This plaque contained a dense core of $A \beta$ fibrils, giving it a characteristic star-shape appearance. Individual $A \beta$ fibrils extended from the core (figure 4.7) in a flame-like fashion. Processes of microglial cells were distributed between the $A \beta$ fibrils (figure 4.6 and 4.7 yellow asterisks). Many of the $A \beta$ fibril-filled invaginations were in the close proximity to rough endoplasmic reticulum (figure 4.6, 4.7). Presumed astroglial processes were also observed surrounding the plaque core (figure 4.6, 4.7). These processes have a lighter appearance and lack rough endoplasmic reticulum (Masliah et al., 1996; Dikranian et al., 2012). 
Surrounding the amyloid core is a corona of dystrophic neurites. Thus, this type of plaque is referred to as a "neuritic plaque" (figure 4.8). These dystrophic neurites contain dense lamellar bodies and vacuoles.

\section{Ultrastructure of plaques in TgSwDI mice}

We next examined the ultrastructure of plaques in tissue obtained from 13 month and 20 month old TgSwDI mice. In this model, neuritic plaques were primarily distributed in perivascular areas, with accumulations of $\mathrm{A} \beta$ observed in the endothelial cells making up vessel walls, and microglia and dystrophic neurites surrounding the vessels (Figure 4.9).

The classification of microglial cells and their processes as well as other types of cells seen in the plaque periphery was based on careful comparison with the microscopic characteristics of similar cells described in previous investigations (Stalder et al., 1999; Wegiel et al., 2004; Van Nostrand et al., 2005; Dikranian et al. 2012). As described in previous studies of neuritic plaques, microglial cells are distributed in the immediate proximity of the amyloid core. The cytoplasm and nuclei of microglial cells are darker than that of neurons. Microglial cells contain abundant rough endoplasmic reticulum and occasional mitochondria (Figures 4.10, 4.11). Occasionally, some cells with very dark nuclei and very dense cytoplasm were found near the site of amyloid pathology (figure 4.12). These cells are presumed to be dystrophic or degenerating microglia cells based on the appearance of the nucleus and cytoplasm filled with vacuoles and other inclusion bodies.

The mossy fiber region of the hippocampus, which, as described above, stains densely with IB4, rarely exhibited typical neuritic plaques. Instead, abundant microglial processes filled with $\mathrm{A} \beta$ fibrils were observed to extend between the neurites with little extracellular amyloid (figure 4.13). In the frontal cortex, we occasionally observed corelike $\mathrm{A} \beta$ accumulations inside microglia or neurons that were not associated with blood 
vessels (figure 4.14). Dystrophic neurites and neurons with amorphous inclusions resembling lipofuscin granules and vacuoles were also observed in areas in which there were no discernible amyloid deposits (Figure 4.15).

The dystrophic neurites were predominantly filled with dense accumulations of intracellular debris such as cytoplasmic membrane and myelin, autophagic vacuoles and degenerating mitochondria (Terry et al., 1963, Masliah et al., 1996; Dikranian et al., 2012). Mitochondrial changes in the neuritic processes ranged from mitochondrial clumping and gradual degeneration to complete absence of distinct organelle features (figure 4.16). The neurites in the latest stages of degeneration were the most electron dense, due to increases in dense lamellar bodies and decrease in surrounding cytoplasm. The majority of dystrophic neurites were unmyelinated, although some myelinated dystrophic axons were present (figure 4.17). In some cases neurites were only partially myelinated (Figure 4.17). In hippocampus, the degenerating neurites exhibited electrondense nuclei and axoplasma (figure 4.16E). Degenerating axon terminals were also observed. These terminals contained electron dense accumulations of synaptic vesicles (figure 4.18).

Finally, some core-like plaque structures were identified inside neuronal processes with adjacent bundles of fibrils resembling neurofibrillary tangles (figure 4.19) (Huang et al., 2001; Ramsden et al., 2005). The origin of these fibrils is unclear, as they could be fibrils of $\mathrm{A} \beta$ or condensed neurofilaments. Similar fibril bundles were observed in the molecular layer of dentate gyrus (figure 4.20).

\section{Discussion}

\section{Plaque progression}

The progression of microglial involvement and the ultrastructure of pathological changes in a triple transgenic mouse model of $\mathrm{AD}$ were investigated in this study. First, 
we have shown that Ib4 labeling of activated microglia is a useful tool for investigating the pathological progression of $\mathrm{AD}$. The co-localization of staining for $\mathrm{A} \beta$ and activated microglia suggests that $\mathrm{A} \beta$ formation and deposition directly or indirectly activate dormant microglia and attract them to sites of fibrillar amyloid accumulation (Stalder et al., 1999; Wegiel et al., 2004; Nagele et al., 2004). However, there are many questions that remain unanswered. The most important question is why these pathological changes have such a specific site of onset in the brain. Regardless of species or the type of mutation initiating plaque formation, pathological changes are initiated in similar areas and progress in a defined sequence with aging (Braak and Braak, 1991; Van Nostrand et al., 2004; Thal et al., 2001, 2006).

In the TgSwDI model we used, the progression of plaque formation in the brain followed the general course described for human $\mathrm{AD}$ plaque progression. We observed sequential plaque formation that started in the subicular area and spread to involve the majority of the hippocampus and the cortex as the animals aged. Why the subiculum/CA1 and enthorhinal cortex are particularly prone to the development of pathology is still unclear. In the TgSwDI used in this study the activated microglia were mostly located in the alveus layer of the hippocampus following activation in the subiculum. The alveus layer contains a large number of myelinated axons coming from pyramidal cells of the subiculim and other parts of hippocampus to the fimbria/fornix, the major output tract for hippocampus (Knowles, 1992). In addition, dense distributions of $A \beta$ and microglia predominated in the mossy and Schaeffer fibers, major incoming excitatory pathways. This suggests that, as animals age, the development of pathology is related to areas in which there are abundant excitatory synaptic connections. The various mechanisms of excitotoxicity (reviewed in detail by Kumar et al., 2013), are considered to be possible triggers of AD pathology (Chen et al., 1992; Chen and Lipton, 1997). Moreover, one of the two medications available as a treatment option 
for $\mathrm{AD}$ patients is memantine, a N-methyl-d-aspartate receptor (NMDAR) antagonist which is thought to effectively block the neurotoxicity of glutamate, slowing neuronal loss and cognitive deterioration (Chen and Lipton, 2006; Hellweg et al., 2012; Cavalli et al., 2013). Another hypothesis regarding the initiation and progression of $A \beta$ accumulation within certain hippocampal areas is related to the abundant zinc storage in the areas where plaques develop first. Diverse mechanisms have been proposed that could explain why changes in zinc levels may lead to the plaque formation (Paoletti et al., 2009). Future experiments will be necessary to investigate those possibilities.

\section{Plaque ultrastructure}

Although many histopathological characteristics of the plaques observed in this study were very similar to the well known changes in humans and other mouse models of $\mathrm{AD}$, there were several unique morphological differences in the TgSwDI mice. Despite thorough investigation, we were unable to find dense cores of extracellular amyloid in the subiculum, mossy fiber area or cortical parenchyma resembling the ones that we observed in the Tg2576 mice, even though the microglial cells and $A \beta$ staining were obvious in those areas in the TgSwDI mice. The propensity of altered $A \beta$ fibrils produced after Dutch and Iowa mutations to accumulate near or in the walls of brain vasculature has been previously described (Van Nostrand et al., 2004, 2005). Our careful ultrastructural examination of areas densely stained with Ib4 suggests that in TgSwDI mice the perivascular depositions of $A \beta$ may be analogous to the dense cores of neuritic plaques. Indeed, most of the pathological elements of $\mathrm{AD}$ were seen in the vicinity of the vessels (figures 4.9, 4.10, 4.11), including processes of activated microglia and dystrophic neurons.

The reason why there is such a strong affinity for $A \beta$ fibrils to accumulate around the cerebral vasculature may lie in the type of $A \beta$ fibrils produced, and the conformational alterations that are produced by the mutations (Van Nostrand et al., 
2001, 2006; Herzig et al., 2004; Baumketner et al., 2008; Meredith et al., 2009; Chen et al., 2011). The increased ratio of $A \beta 40 / A \beta 42$ in the plaques of Dutch and Iowa mutants leads to the predominant accumulation of $\mathrm{A} \beta 4 \mathrm{O}$ in the vasculature. Despite the solubility of $\mathrm{A} \beta 40$, the several fold increase in its concentration in TgSwDI causes its retention in the brain (Van Nostrand et al., 2004). Lin et al., (2013) suggested that the $A \beta 40 / A \beta 42$ ratio determines the type of plaque formed, with increased $A \beta 40$ primarily responsible for diffuse plaque formation. Shifting the $A \beta 40 / A \beta 42$ ratio toward more $A \beta 42$ formation, by introducing additional mutations (for example, mutated presenelin-1), redistributes amyloid pathology from vascular to more parenchymal (Levy et al., 2006; Herzig et al., 2004; Chen et al., 2011). Interestingly, despite the pronounced perivascular pathological changes we observed in the TgSwDI mice, the vascular lumen were not occluded and the overall vascular shape was preserved in the most affected vessels (figure 4.9, 4.11). This suggests mechanisms of amyloid retention other than simple mechanical occlusion by A $\beta$ (Van Nostrand et al., 2004; Deane et al., 2004).

The abundant presence of the diffuse type of plaque (i.e. plaque that are devoid of both a dense core and surrounding neurites and inflammatory types of cells), was previously shown in this model (Van Nostrand et al., 2004, 2005) and is in accordance with the types of plaques seen in carriers of the Dutch and Iowa mutation (Levy et al., 1990; Yamaguchi et al., 2000; Grabowski et al., 2001; Van Nostrand et al., 2004). However, this type of plaque characteristic has mainly been described based on the cloud-like appearance of $A \beta$ staining observed with the light microscope, and it is extremely difficult to discern subtle collections of $A \beta$ fibrils at the electron microscopic level without special immunogold staining (Yamaguchi et al., 2000).

The presence of diffuse $A \beta$ fibrils exclusively would not explain the symptoms of $\mathrm{AD}$, which are more related to neuronal degeneration and loss. The abundant staining of activated microglia co-localized with $\mathrm{A} \beta$ staining that we observed in this study implies 
an interaction between amyloid deposition and immunological reactions. Occasionally, we found $A \beta$ accumulations inside the microglial processes in the areas remote from microvasculature, suggesting attempts to phagocytose and isolate the $A \beta$ (figures 4.14, 4.15).

The ultrastructure of degenerating neurites was similar to that observed in human brains and other models of AD (Terry et al., 1963; Masliah et al., 1996; Perl, 2010; Dikraian et al., 2012). Axons gradually lost their internal structure, showing clumps of mitochondria or complete autophagocitosis and loss of myelin sheaths. Some synapses could be still visualized despite the gross pathology of presynaptic axons, but these preserved synapses were rarely found in the areas of the intense pathology. This synaptic wasting is considered to be one of the strongest correlates of cognitive deterioration (Terry et al., 1991; DeKosky et al., 1996; Scheff and Price, 2003; Venkitaramani et al., 2007; Reddy and Beal, 2008; Bayer and Wirths, 2010). The loss of mitochondrial function is also implicated in synaptic pathology through increased oxidative stress and other mechanisms (Reddy et a., 2010; Quiroz-Baez et al., 2013). The compaction of this organelle and loss of normal cytoskeletal characteristics by the axons as well as Wallerian degeneration imply an impairment of axonal transport (Wirths et al., 2006; Buki and Povlishock, 2006; Dikranian et al., 2012) that likely contribute to the overall neuronal dysfunction.

The role of microglia in the pathology of $\mathrm{AD}$ is a topic of intense debate. In TgSwDI mice, microglia appear to be present at all sites where $A \beta$ staining is found. It is not understood what causes the microglial activation in $\mathrm{AD}$. It has been proposed that $\mathrm{A} \beta$ itself induces a chemotaxis of microglia (Huang et al., 2010). Microglia have been found to internalize A $\beta$ fibrils through special receptors (Paresce et al., 1996; Liu et al., 2005, 2012). Meyer-Luehmann et al (2008) showed that plaques can form within 24 hours, with activated microglia are attracted to the plaque within 1-2 days, followed by 
progressive neuritic changes over the next several days or weeks. The size of the plaque surrounded by microglia remains constant over time which presumes a failure of microglia to efficiently phagocytose amyloid. However, the reason for this presumed failure is not well understood (Meyer-Luehmann et al., 2008; Neumann et al., 2013). Cultured microglia in vitro did not show significant $\mathrm{A} \beta$ degradation abilities. The cells also showed some slow release of intact fibrils after initial uptake (Chung et al., 1999). This could potentially mean that degradation of $\mathrm{A} \beta$ by microglial cells is naturally ineffective and initial unsuccessful attempts in clearing $A \beta$ fibrils is followed by their release and further build up in the extracellular space.

In our study, we found many microglial cells at the sites of $A \beta$ deposition clearly showed amyloid masses inside their processes, but with no clear signs of phagocytotic activity, such as vacuoles, lysosomes or any inclusion bodies. In contrast, microglial cells that were close to dystrophic neurites showed signs of phagocytosis (figure 4.21), in accordance to previous studies (Terry et al., 1964; Wegiel and Wisniewski, 1990; Stalder et al., 1999). The participation of microglia in phagocytosis of axonal and myelin debris of dystrophic neurites (Tanaka et al., 2009; Hosmane et al., 2012) is very important. Many examples of axonal Wallerian type of degeneration and loss of myelination in TgSwDI and other models imply a great amount of degrading myelin is cleared by the microglia. Myelin proteins impede axonal regeneration and repair and have also been shown to inhibit phagocytosis by microglia (Wang et al., 2002; Gitik et al., 2011; Neumann et al., 2013). To further complicate matters, it has been found that myelin clearance is very inefficient after Wallerian type of degeneration in the central nervous system (Gaudet et al., 2011; Neumann et al., 2013). Thus, the promotion of microglial activity could be beneficial for improved neural functioning in AD. On the other hand, persistent microglial activation could potentially cause chronic inflammation and neurodegeneration through various cytokines and reactive oxygen species (McGeer and 
McGeer, 1995; Rogers et al., 1996; Stalder et al., 2001). Also, investigations of the ineffective involvement of astrocytes in the clearing of $\mathrm{A} \beta$ and cellular debris (Nagele et al., 2004) are interesting in light of the fact that we found evidence of the presence of astrocytic processes in and around plaques in the TgSwDI mice.

In summary, our investigation of TgSwDI mice confirms most of previously described histopathological features of this unique $\mathrm{AD}$ model. It was estimated that direct contact with capillaries was found in $91 \%$ of amyloid plaques in human (Kawai et al., 1990; Nagele et al., 2004). Recent studies suggested that dementia shows a better correlation with microvascular $A \beta$ accumulation than parenchymal $A \beta$ accumulation (Thal et al., 2003; Atterns and Jellinger, 2004). Failure of anti-amyloid therapy to improve cognitive functioning in human trials (Sperling et al., 2012) shifted the previous "amyloid cascade" hypothesis toward searches for other causes of AD etiopathogenesis. In this case, TgSwDI mice represent a suitable model for investigation of the microvascular aspects of $\mathrm{AD}$ pathology. 
Figure 4.1 Confocal image of $A \beta$ plaques and associated microglia in 13-month old TgSwDI. (A) 4G8-staining of cortical plaques for A $\beta$ co-localizes with (B) Ib4 staining for activated microglia. (C) Higher magnification image of the cortical plaque stained for $4 G 8$ co-localizes with (D) Ib4 staining of the same plaque. (E) Superimposed image of (C) and (D), ZnT3-staining co-localize; DAPI-staining reveals dense nuclei of microglia (black arrow) and more lucid nuclei of neurons (white arrow) lying in the vicinity of the plaque. Scale bar in $(A)=10 \mu \mathrm{m}$ and applies to $(B)$. Scale bar in $(C)=5$ $\mu m$ and applies to (D) and (E) 

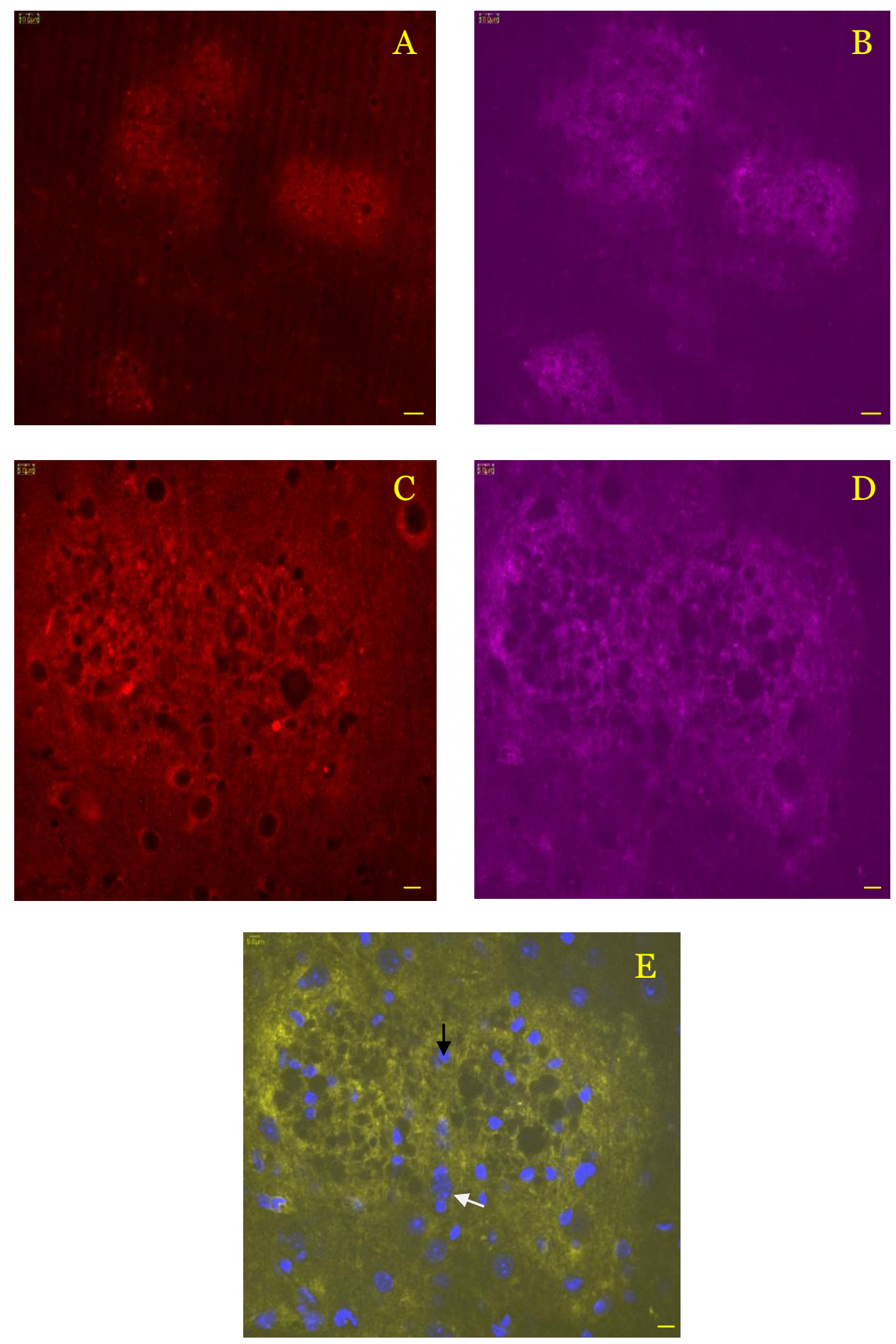
Figure 4.2 Confocal image of mossy fibers $(M F)$ stained for $A \beta(A, C, E)$ with $4 G 8$ and microglia with Ib4 $(B, D, F)$. There is no signal for $4 G 8$ or Ib4 in P21 WT $(A, B)$ and 4month old TgSwDI mice (C, D) noted, but strong signal for both appears in 13-month old TgSwDI and overlap (E, F). Scale bar $(A)$ and $(B)=10 \mu \mathrm{m}$; scale bar $(C)$ and $(D)=$ $50 \mu \mathrm{m} ;$ scale bar (E) and $(B)=20 \mu \mathrm{m}$. 

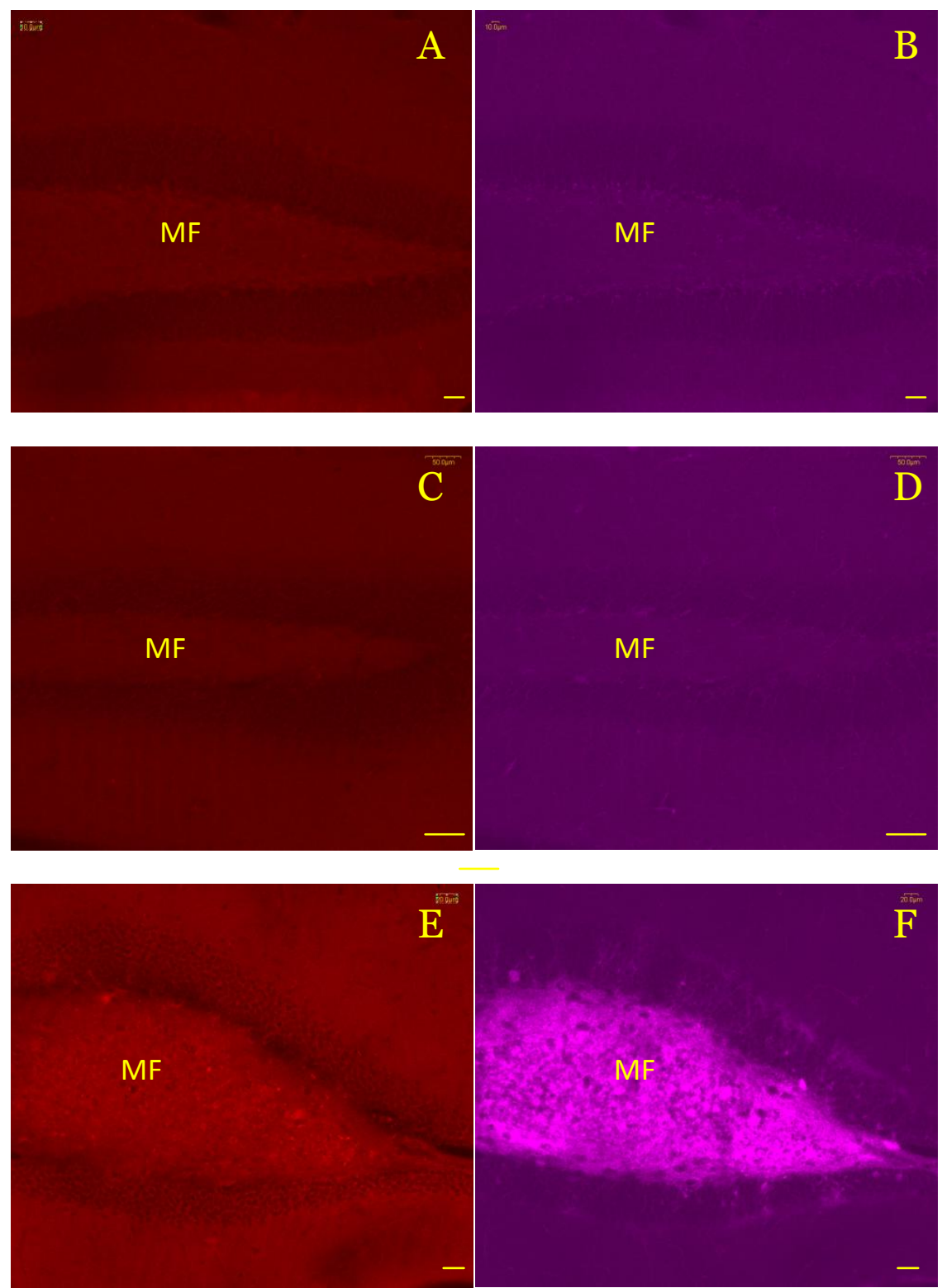
Figure 4.3 Progression of AD pathology in TgSwDI at different ages. (A) P21 WT mouse hippocampus stained for Ib4, no specific areas of labeling is noted. (B) Ib4 staining for activated microglia in 4-month old TgSwDI. The sharply defined clusters of labeled microglia noted (arrow) in subiculum. (C) 6-month old age TgSwDI shows activated microglia spreading to the alveus layer of CA1. (D) and (E) At 14 months of age, TgSwDI shows activated microglia in every area of hippocampus and also in the layer of the cortex just above hippocampus (arrows). (E) Hippocamus of 8-month old TgSwDI stained for $4 G 8$ against $A \beta$ shows that stained areas (dotted areas pointed by arrows) are the same areas that contain labeled activated microglia. Scale bar $(A)=$ 100 m and applies to all other panels 


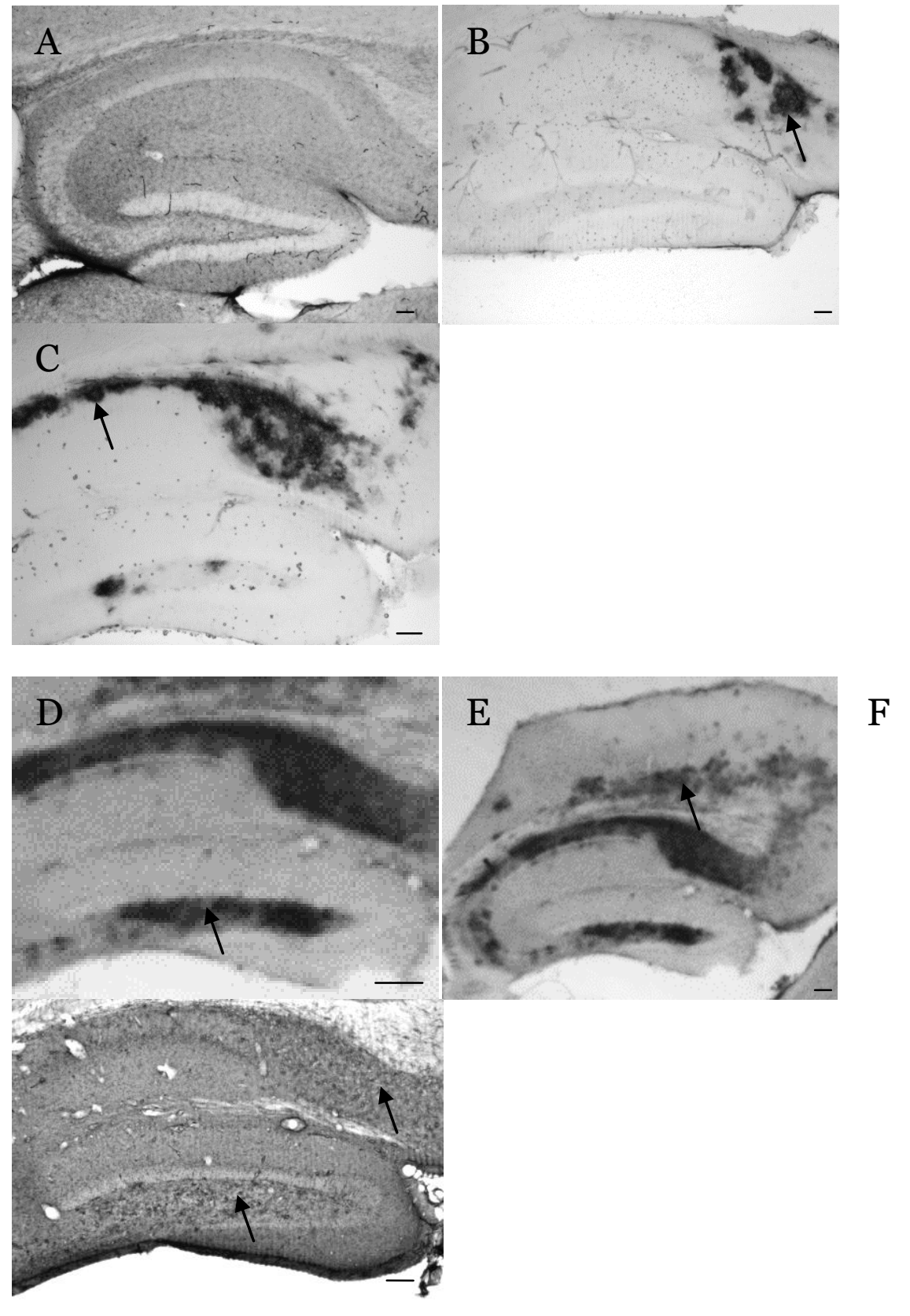


Figure 4.4 Sequential saggital sections of 20-month old TgSwDI stained for Ib4. The plaques are noted in the cortex, including frontal part ( $E$ and $F$ ), in and around hippocampus (A through $F$ ) and striatum (D-F). The inset demonstrates 2O-month old TgSwDI hippocampus after Ib4 staining with the major anatomical areas labeled. MF - mossy fibers; DG - dentate gyrus. Scale bar in $(A-F)=1 \mathrm{~mm}$; scale bar in inset $=250$ $\mu m$ 


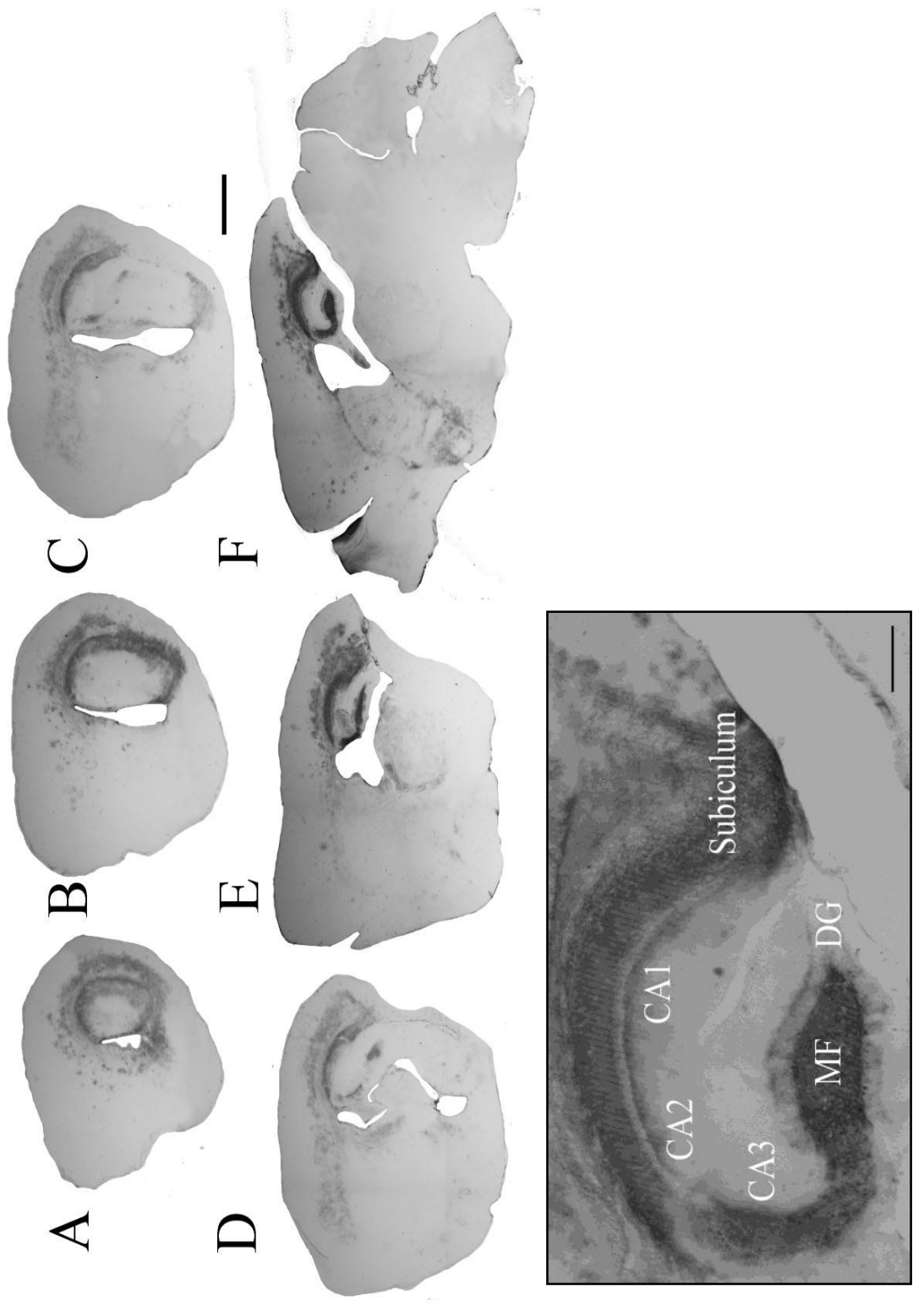


Figure 4.5 Ib4 staining in 2O-month old TgSwDI. Activated microglia is concentrated around the vessels (asterisks) in (A) subiculum; (B) frontal cortex; (C) alveus; (D) olfactory bulb was also dense in Ib4 staining. Scale bar $=10 \mu \mathrm{m}$ 


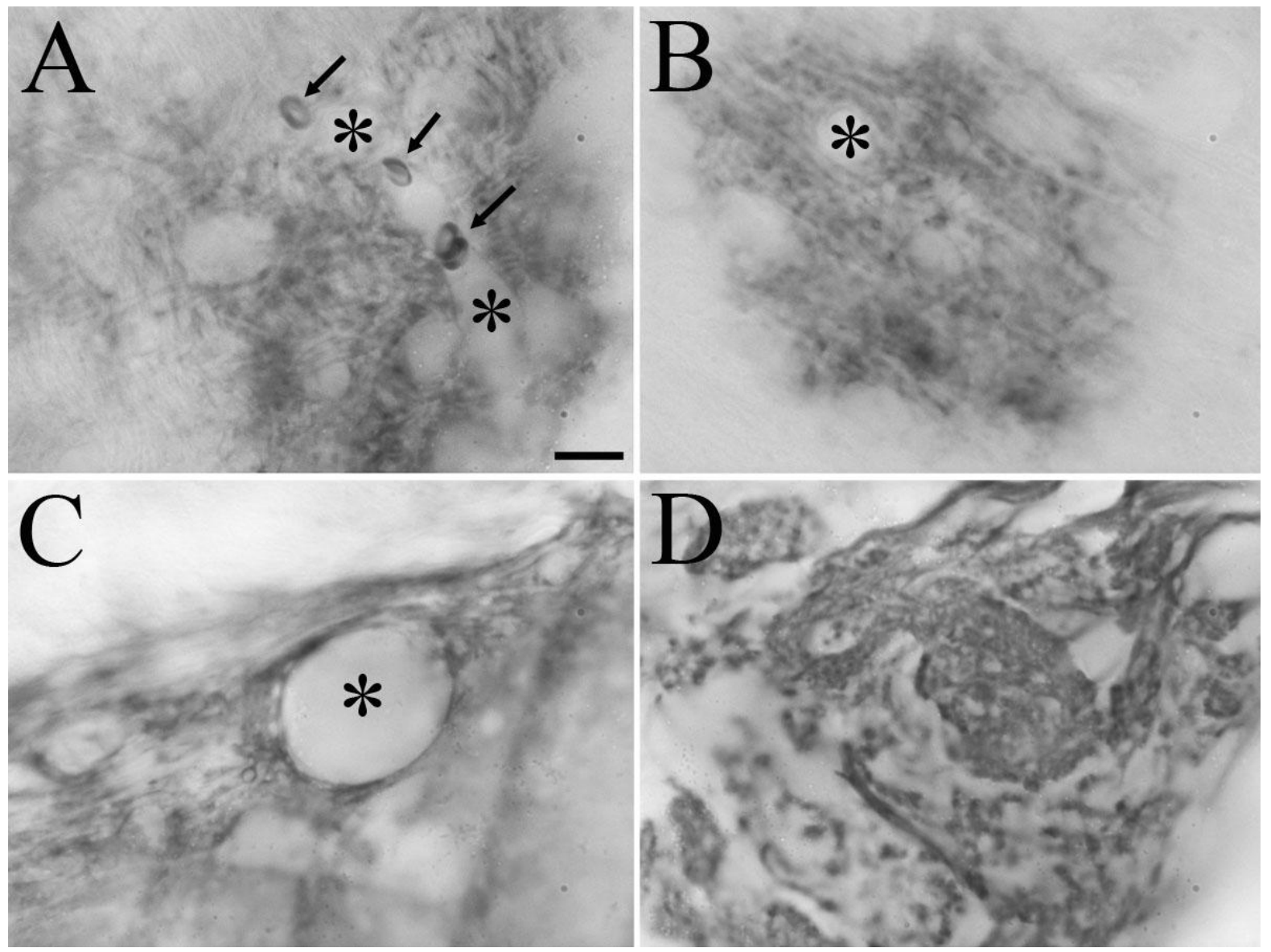


Figure 4.6 Mature plaque core in 24-month old Tg2576. Red asterisk-amyloid core; yellow asterisks - surrounding core microglial processes, black arrows astrocytic processes; white arrow example of amyloid invagination into surrounding microglia; yellow arrow - RER. Inset shows plaque at light microscopic level with red asterisk corresponding to the amyloid dense core shown in the EM picture. Scale bar = $2 \mu m$ 


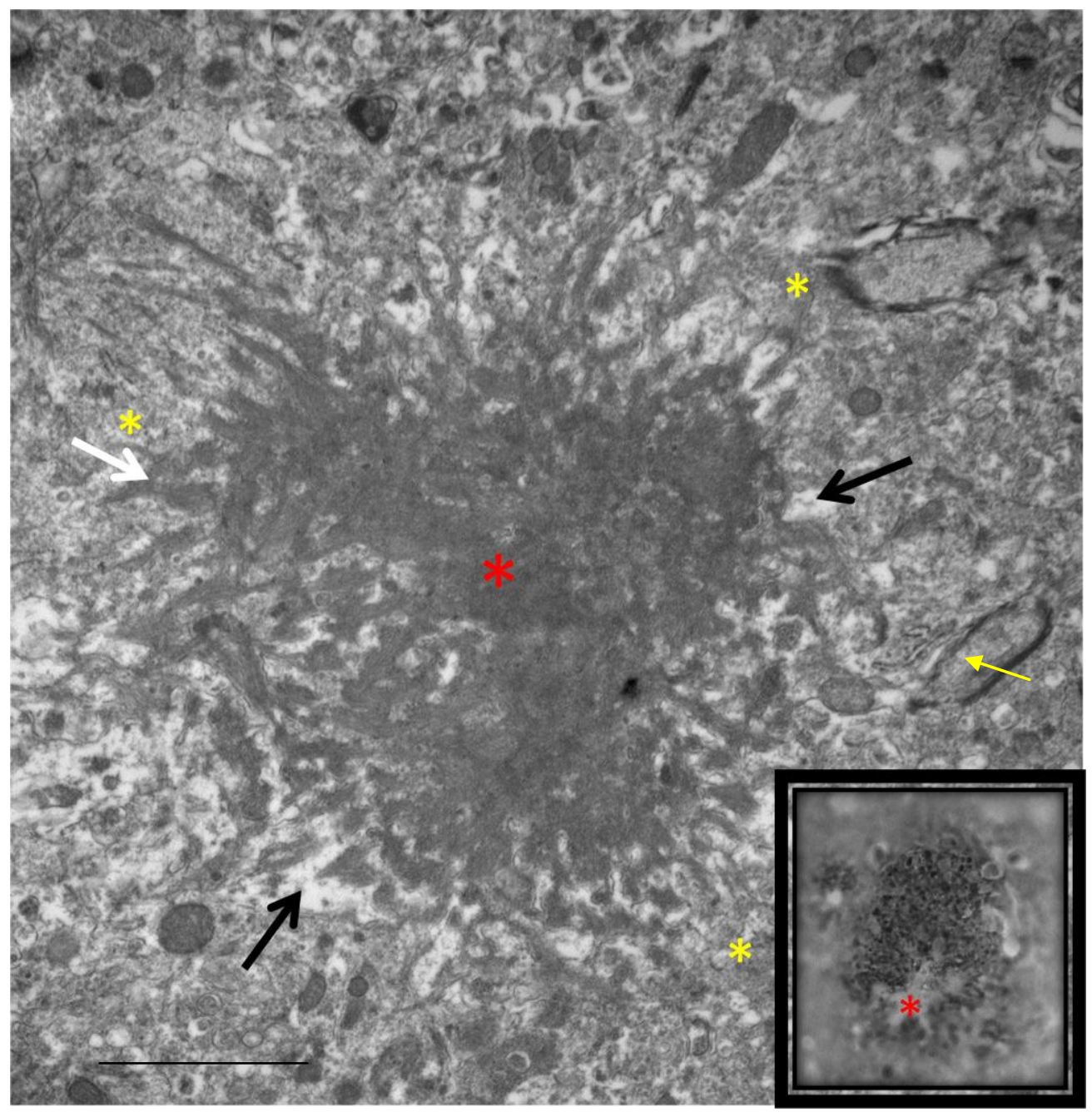


Figure 4.7 Mature plaque core with A $\beta$ fibrils, Tg2576 24-month old. Higher magnification of $A \beta$ fibrils (black arrow) arranged toward the core in microglial process (yellow asterisk). Red - amyloid dense core, black asterisk - astroglial process; yellow arrow - RER. Scale bar $=1 \mu \mathrm{m}$ 


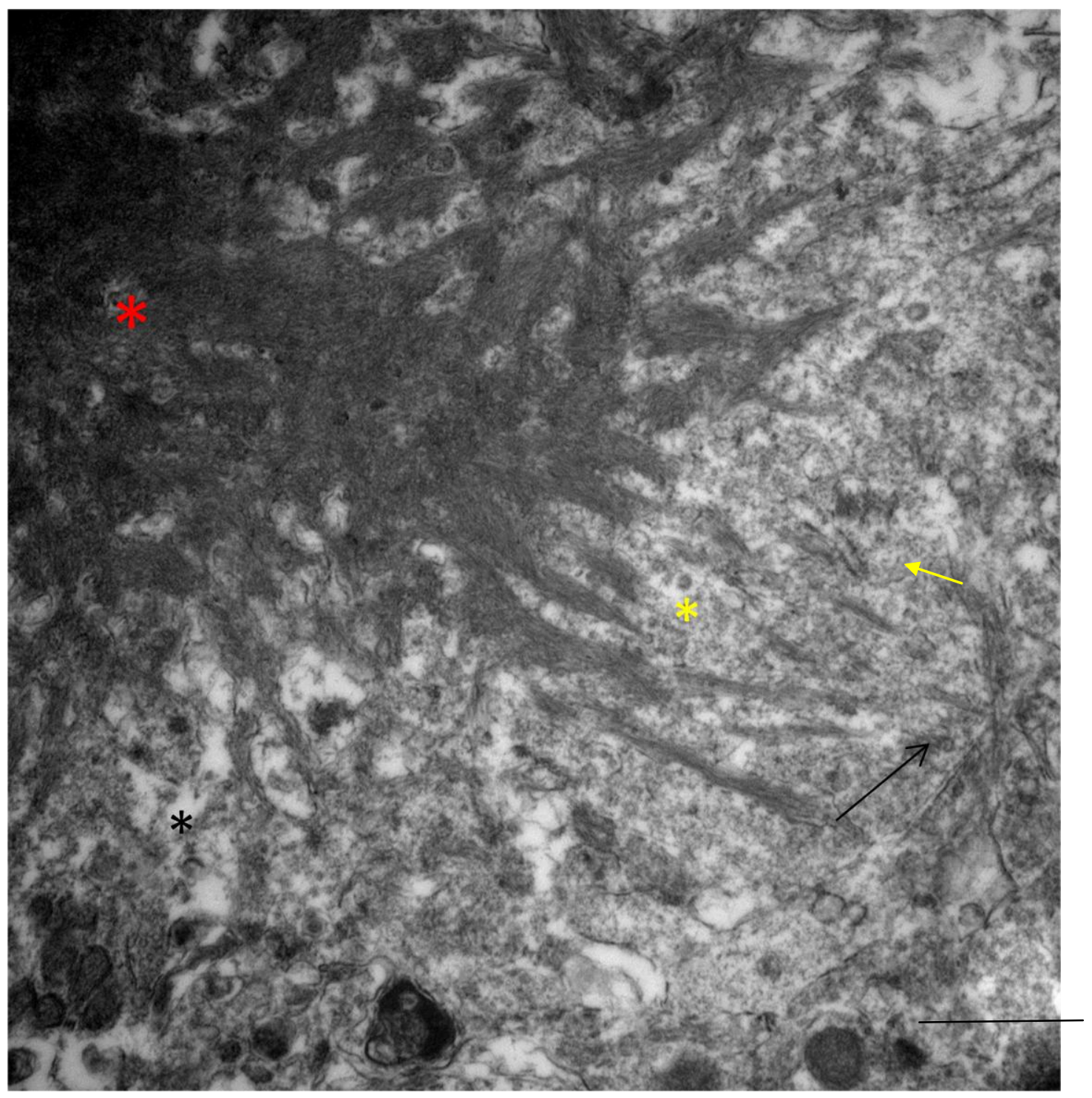


Figure 4.8 Amyloid core periphery Tg2576 24-months old, lower magnification. Red asterisk - amyloid core; white asterisks - microglial processes; yellow asterisks dystrophic neurites in the different stage of degeneration. The dystrophic neurites form corona around dense core. Scale bar $=10 \mu \mathrm{m}$ 


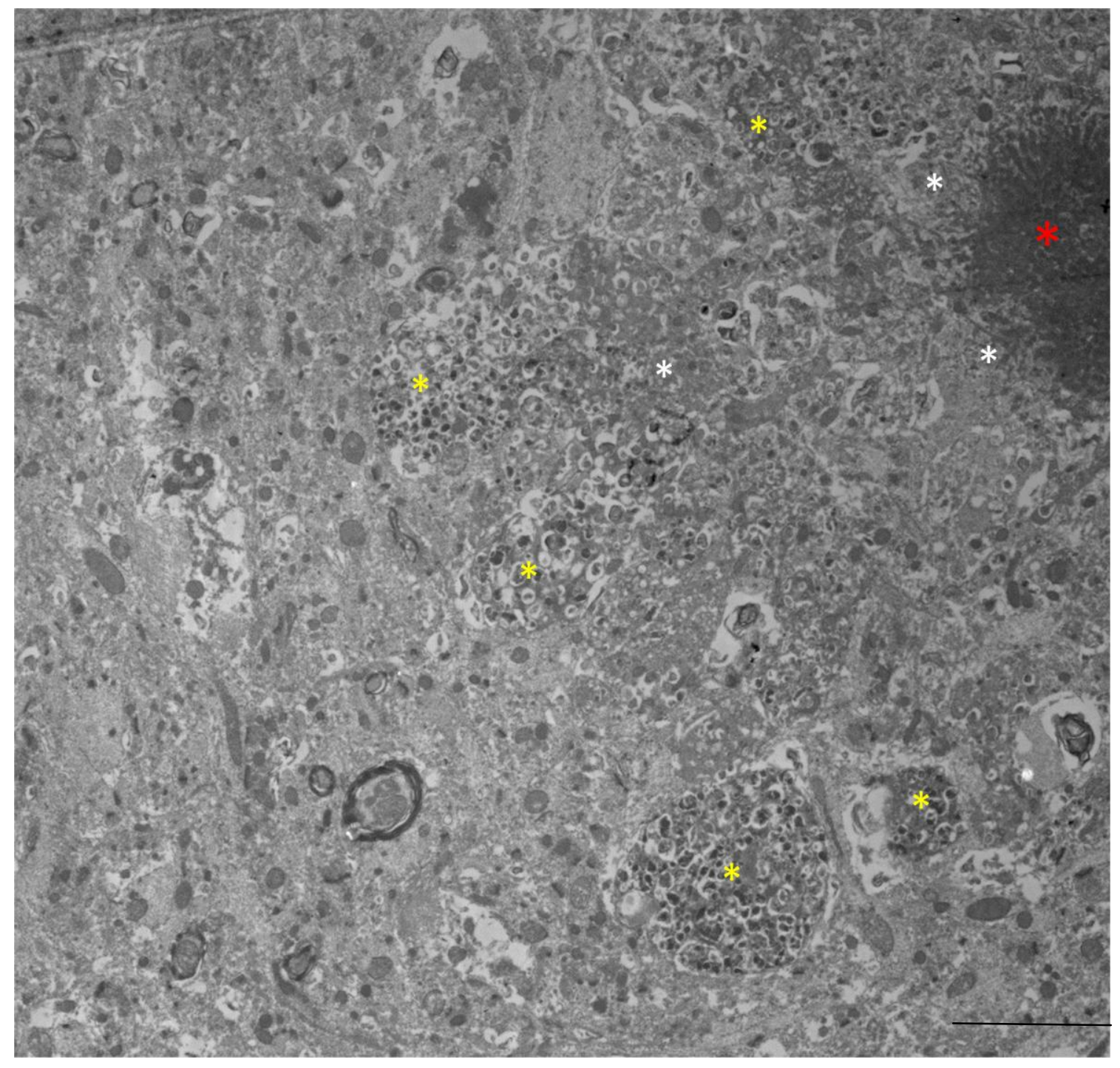


Figure 4.9 TgSwDI 2O-month old perivascular plaque. Red asterisk - A $\beta$ accumulation surrounded by microglial process (yellow asterisk); black arrow - basement membrane; $M$ - microglial nucleus; $L$ - capillary lumen; $N$ - normally appearing neuron; $E$ - endothelial cell. Scale bar $=2 \mu \mathrm{m}$ 


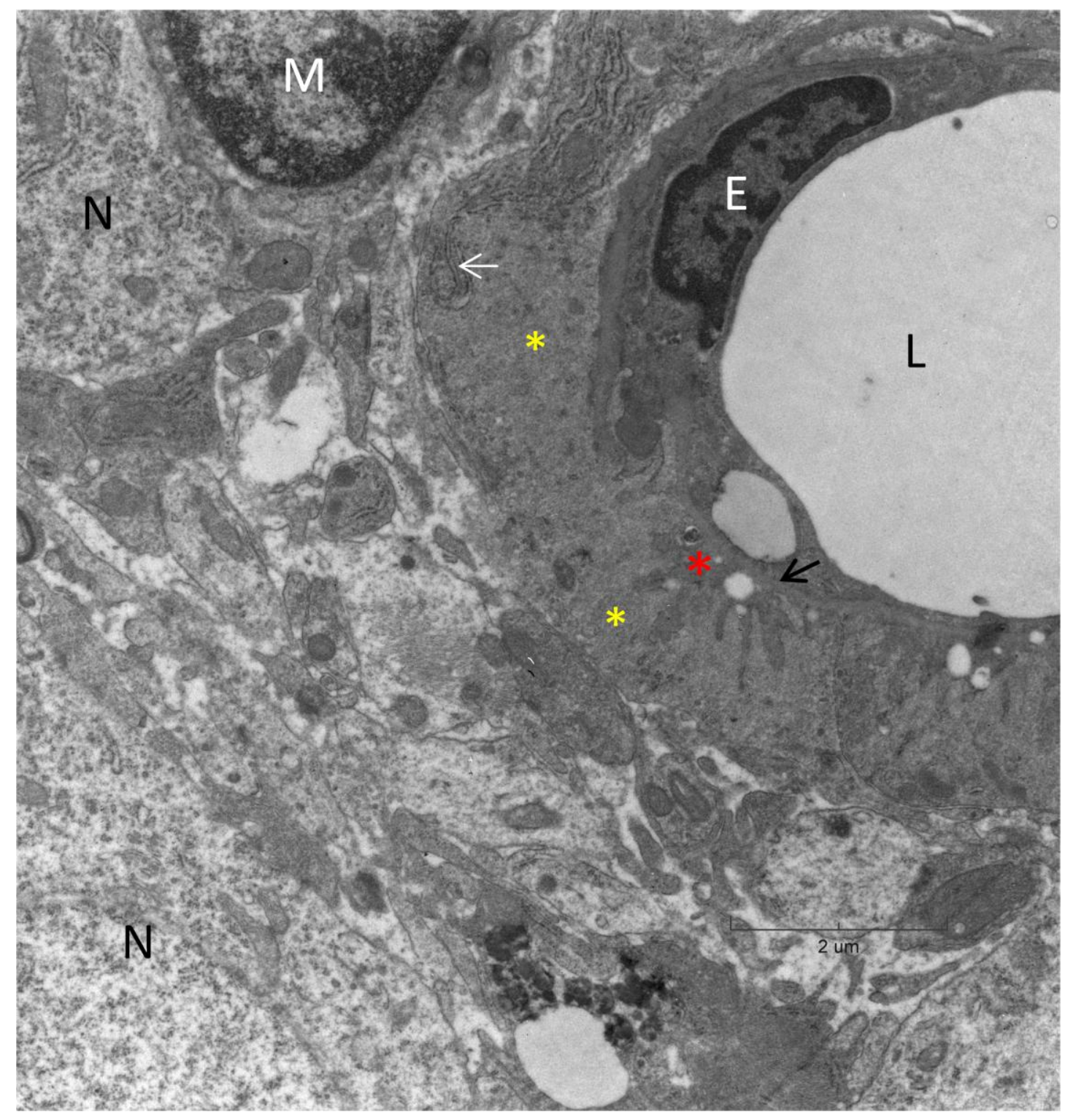


Figure 4.10 TgSwDI 13 month old perivascular plaque. Note A $\beta$ fibrils (red asterisk) form an immediate contact with dystrophic neuron (dn) and surrounded by microglial processes (yellow asterisks). L - capillary lumen; $M$ - microglial nucleus; white arrow - RER with ribosomes; black arrow - basement membrane; yellow arrows mitochondria in microglial cell. Scale bar $=2 \mu \mathrm{m}$ 





Figure 4.11 TgSwDI 2O-month old frontal cortex perivascular plaques. Three microglial cells with multiple amyloid depositions inside surround two affected capillaries. Red asterisks - A $\beta$ accumulations surrounded by microglial processes (yellow asterisks); M- microglial niclei; as - astrocytic processes; L - capillary lumen; $d n$ - unmyelinated dystrophic neurite. Scale bar $=5 \mu \mathrm{m}$ 


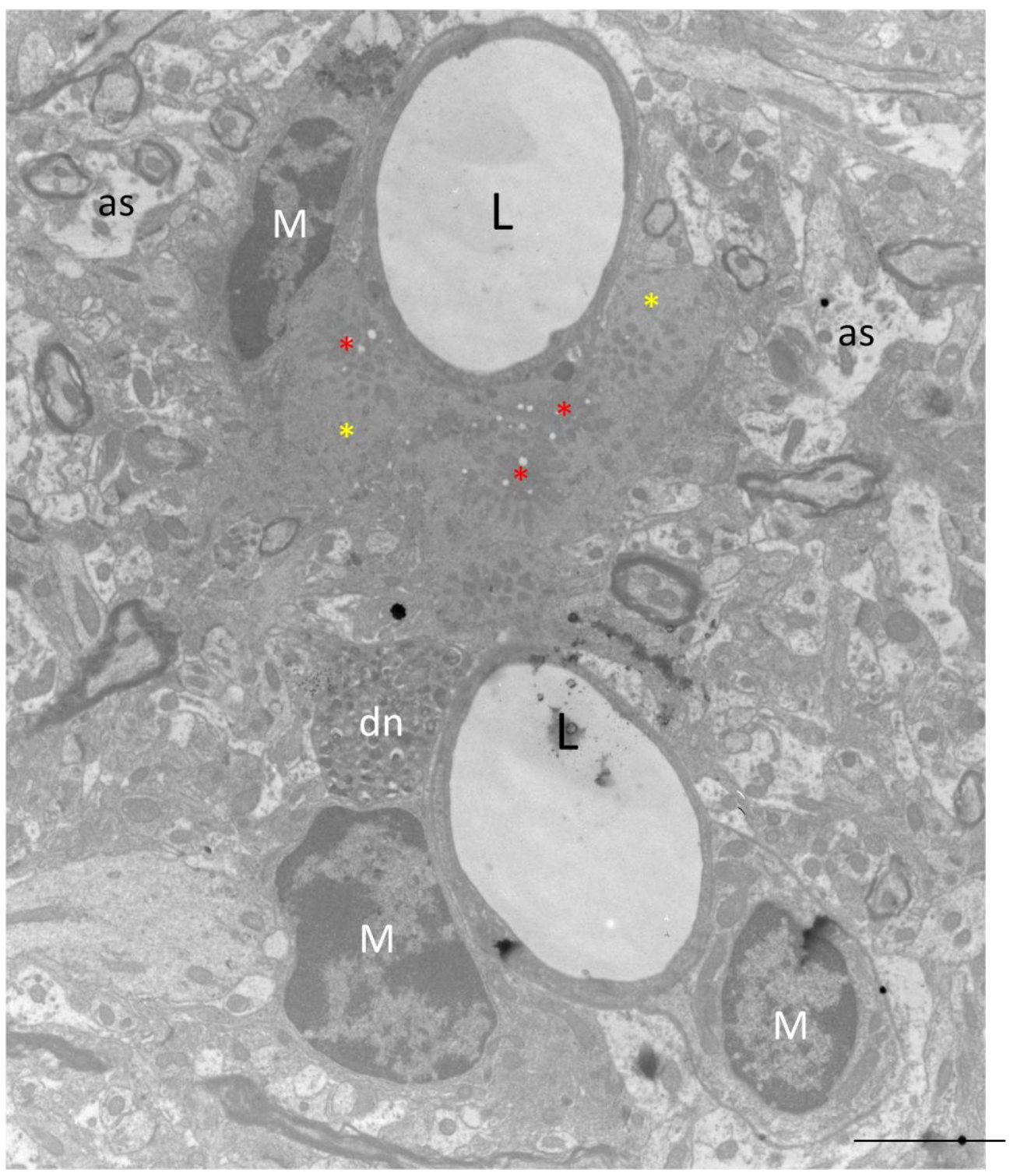


Figure 4.12 Dying microglial cell in TgSwDI 13-month old, cortex. Dark nucleus (M) and cytoplasm (yellow asterisks). Cytoplasm shows signs of phagocytosis: vacuoles (black arrow) and inclusion bodies (white arrow) inside. Dystrophic neuron (dn) and collections of fibrils, presumably of amyloid (red asterisk); Scale bar $=1 \mu \mathrm{m}$ 


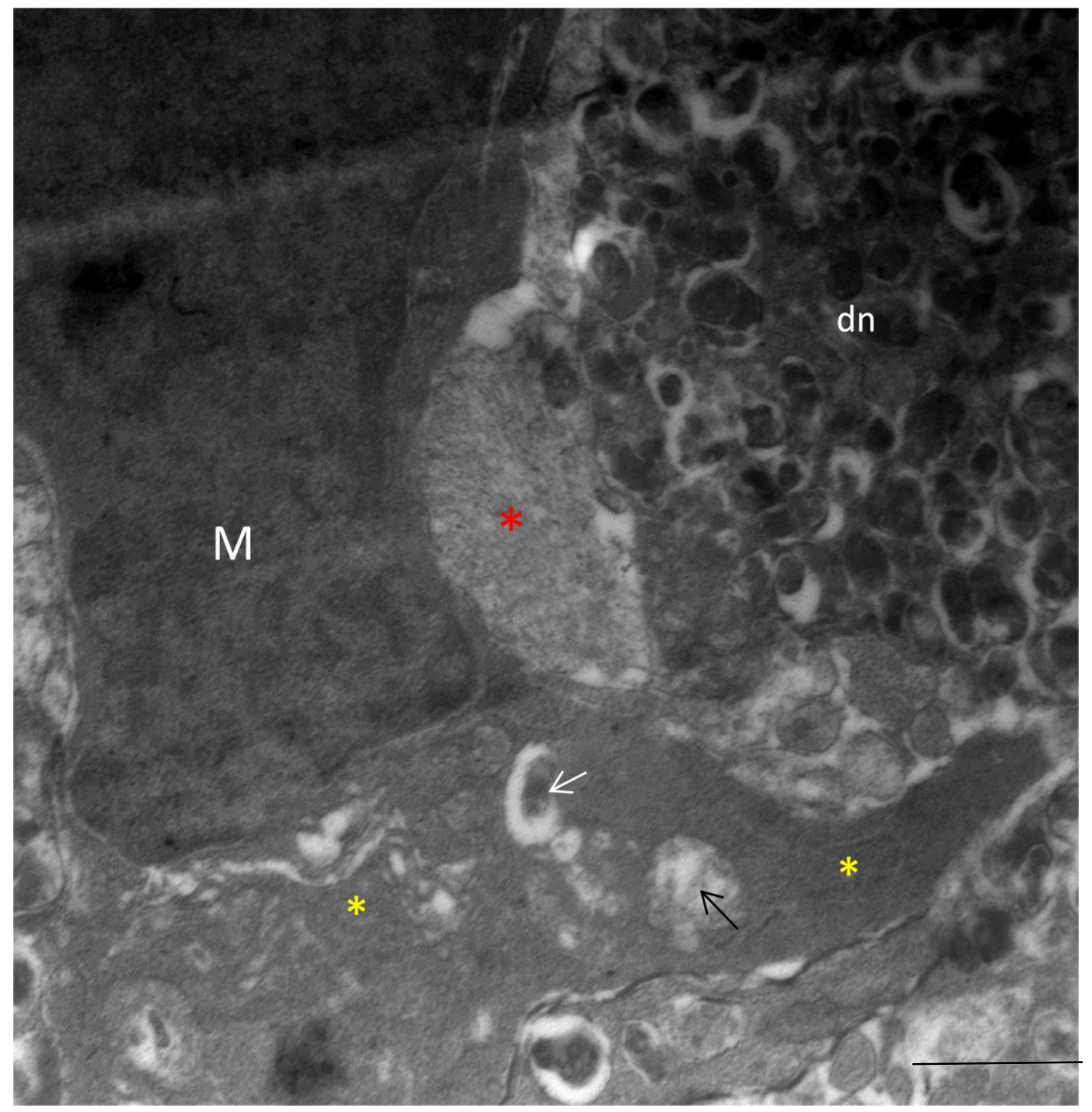


Figure 4.13 Microglial process (M) in mossy fibers of TgAD 13-month old mouse. The process filled with fibrillar material, surrounded by dystrophic neurites (dn). Process contains RER (black arrow) and mitochondria (white arrow). Scale bar $=2 \mu \mathrm{m}$ 


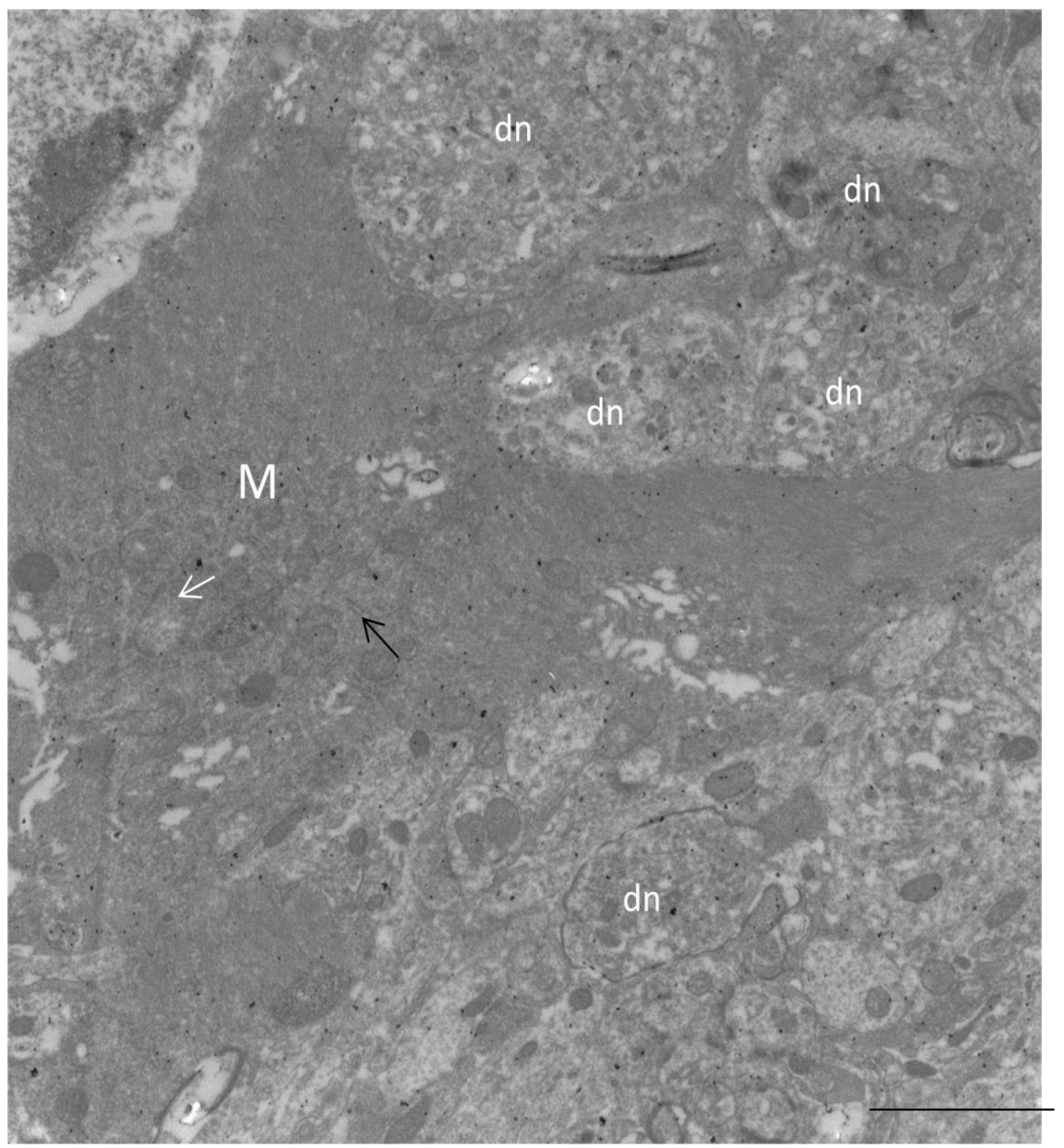


Figure 4.14 Microglial cells (M) in brain parenchyma of frontal cortex in 20-months old TgSwDI filled with (A) multiple or (B) single A $\beta$ depositions (red asterisks) in their processes. Scale bar $=2 \mu \mathrm{m}$ 

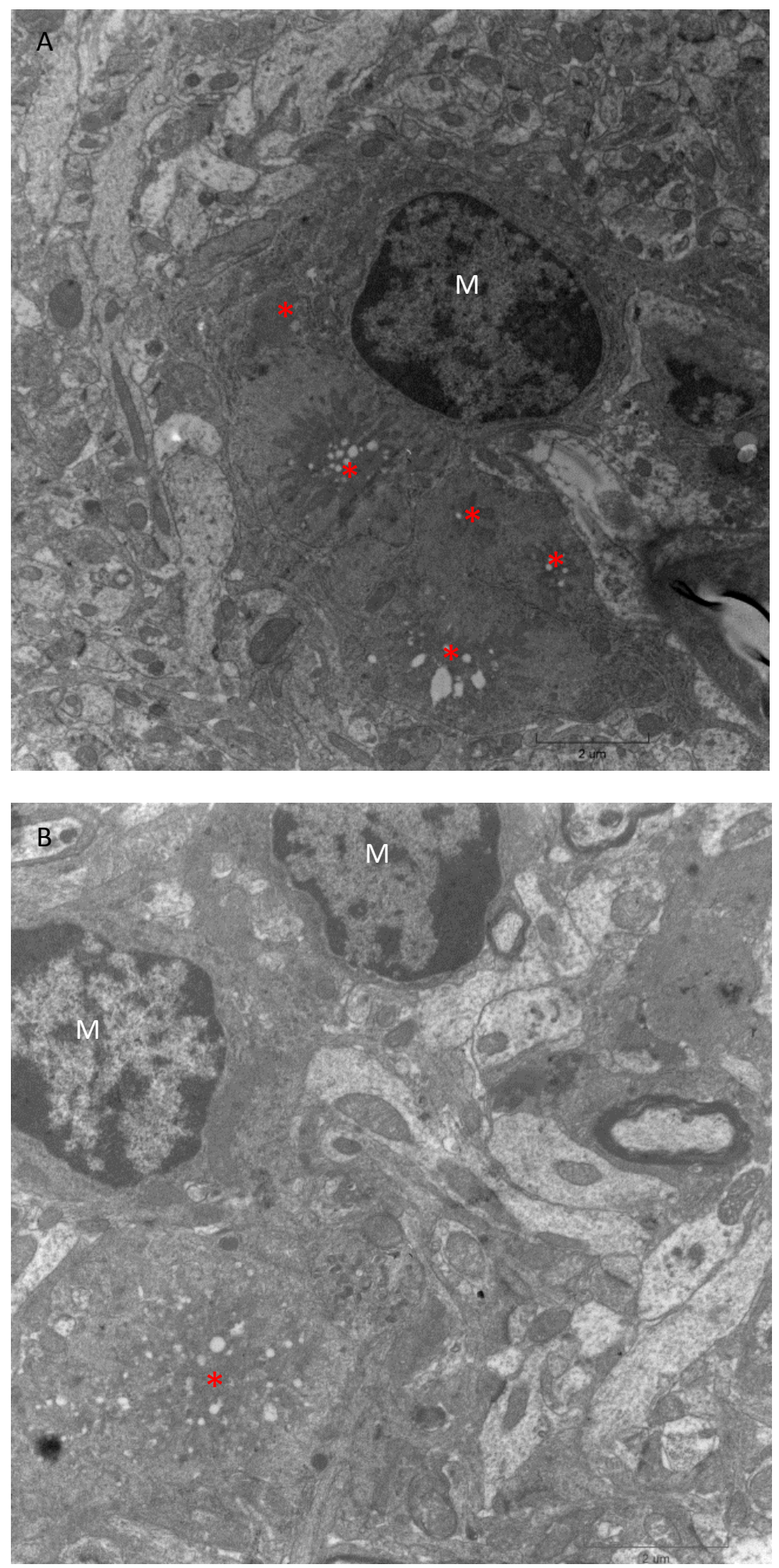
Figure 4.15 Neuron (N) surrounded by microglia (M) in 20-month old TgSwDI frontal cortex. Note the lipofuscin accumulations (white arrows) and vacuoles (black arrow) inside the neuron. Red asterisk shows $A \beta$ accumulation inside microglial process; $d n-$ dystrophic neuron. Scale bar $=5 \mu \mathrm{m}$ 


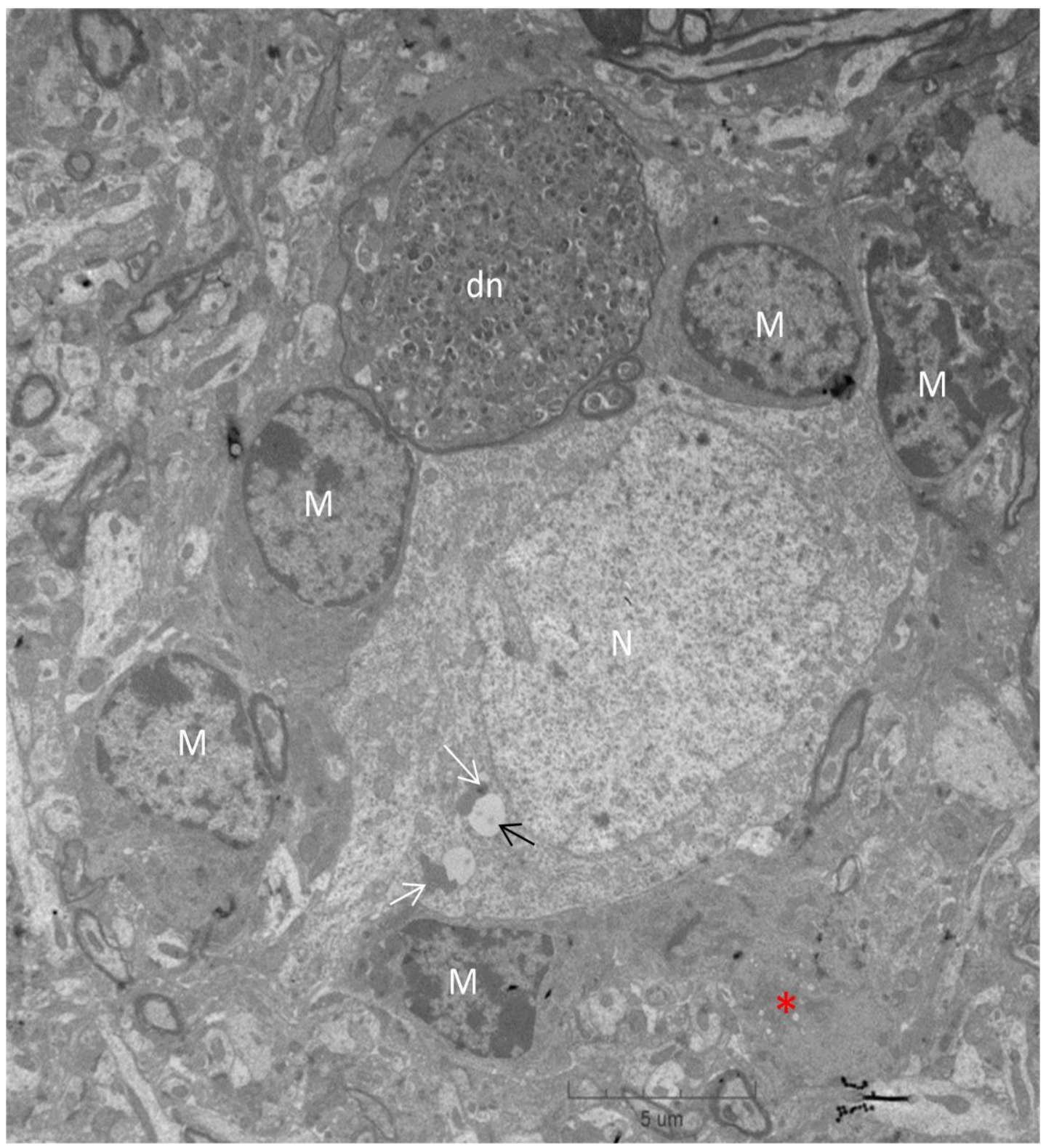


Figure 4.16 Different stages of neurite degeneration, TgSwDI 13-month old. (A) preserved neurofilaments, RER (yellow arrow) and clumping of mitochondria (black arrows); udn - unmyelinated dystrophic neurite; mdn - myelinated dystrophic neurite. (B) Occasional mitochondria are seen (black arrows), areas with neurofilaments are still present; some inclusion bodies (white arrow), dense bodies (green arrow) and vacuoles (yellow arrows) are noticed. (C) No mitochondria are noticed, very little areas with neurofilaments; majority of area filled with dense bodies, inclusion bodies and vacuoles. (D) Late stage of myelinated axon degeneration, filled with dense bodies and vacuoles, no spaces with neurofilaments could be seen. (E) Neuronal body in the initial stage of apoptosis with pyknotic nucleus and dense cytoplasm without any visible organelles. Scale bar (A) $2 \mu$ m and applies to (B, $C$ and D). Scale bar $(E)=5 \mu m$ 


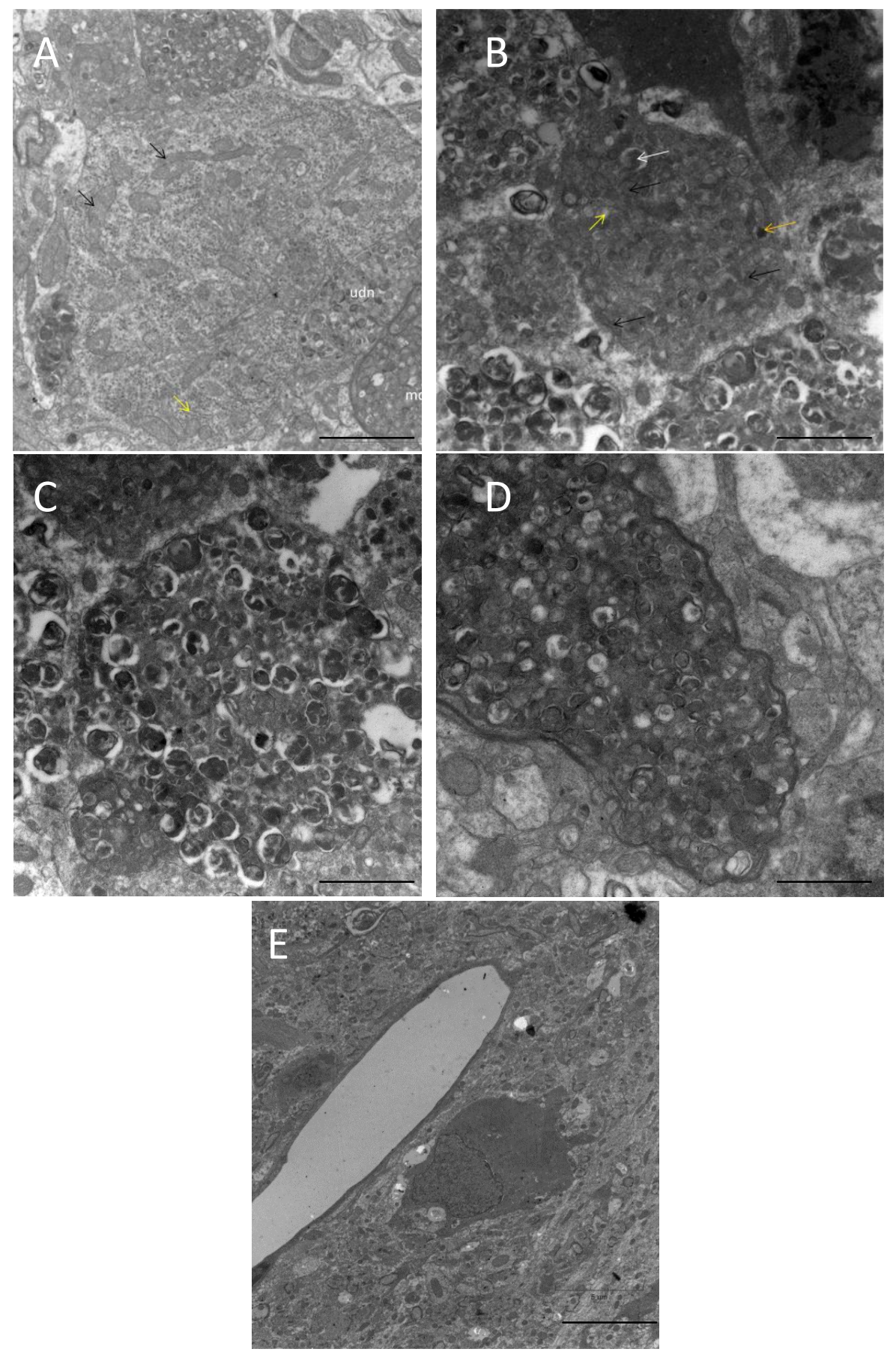


Figure 4.17 Loss of myelination by degenerating axon of TgSwDI 13-month old (A) cortex (B) hippocampus. Black arrows show myelinaed part and white arrows show umyelinated part of the axons; $d n-d y$ strophic neuron. Scale bar $=2 \mu \mathrm{m}$ and applies to ( $A$ and $B$ ) 

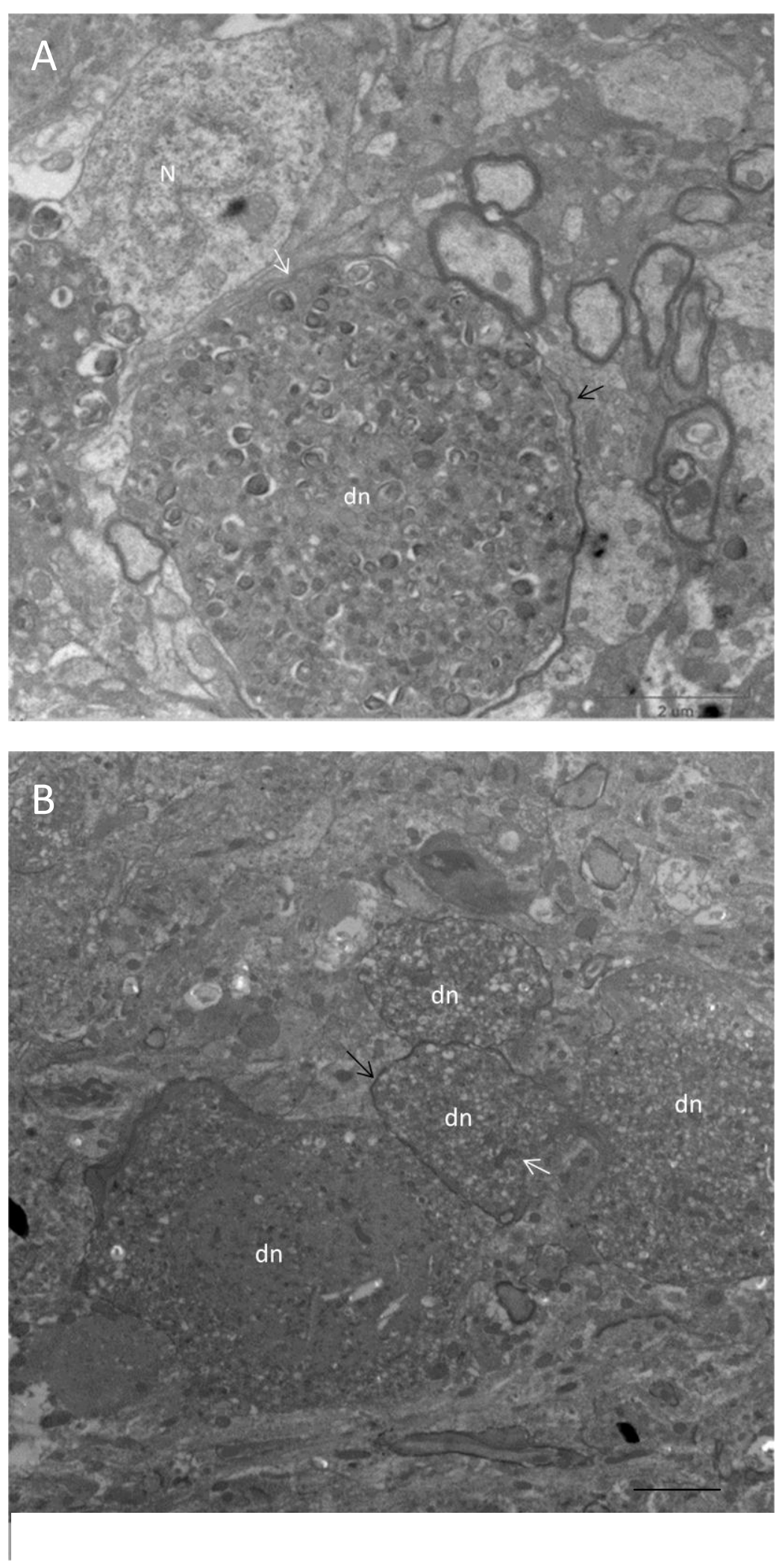
Figure 4.18 Degenerating synaptic terminal in 13-monh old TgSwDI. Terminal (T) of degenerating axon ( $\mathrm{dn}$ ) forms multiple synapses (black arrows) with dendritic spines and has visible dense bodies (yellow arrow) among the normal appearing terminal vesicles. Scale bar $=2 \mu \mathrm{m}$ 


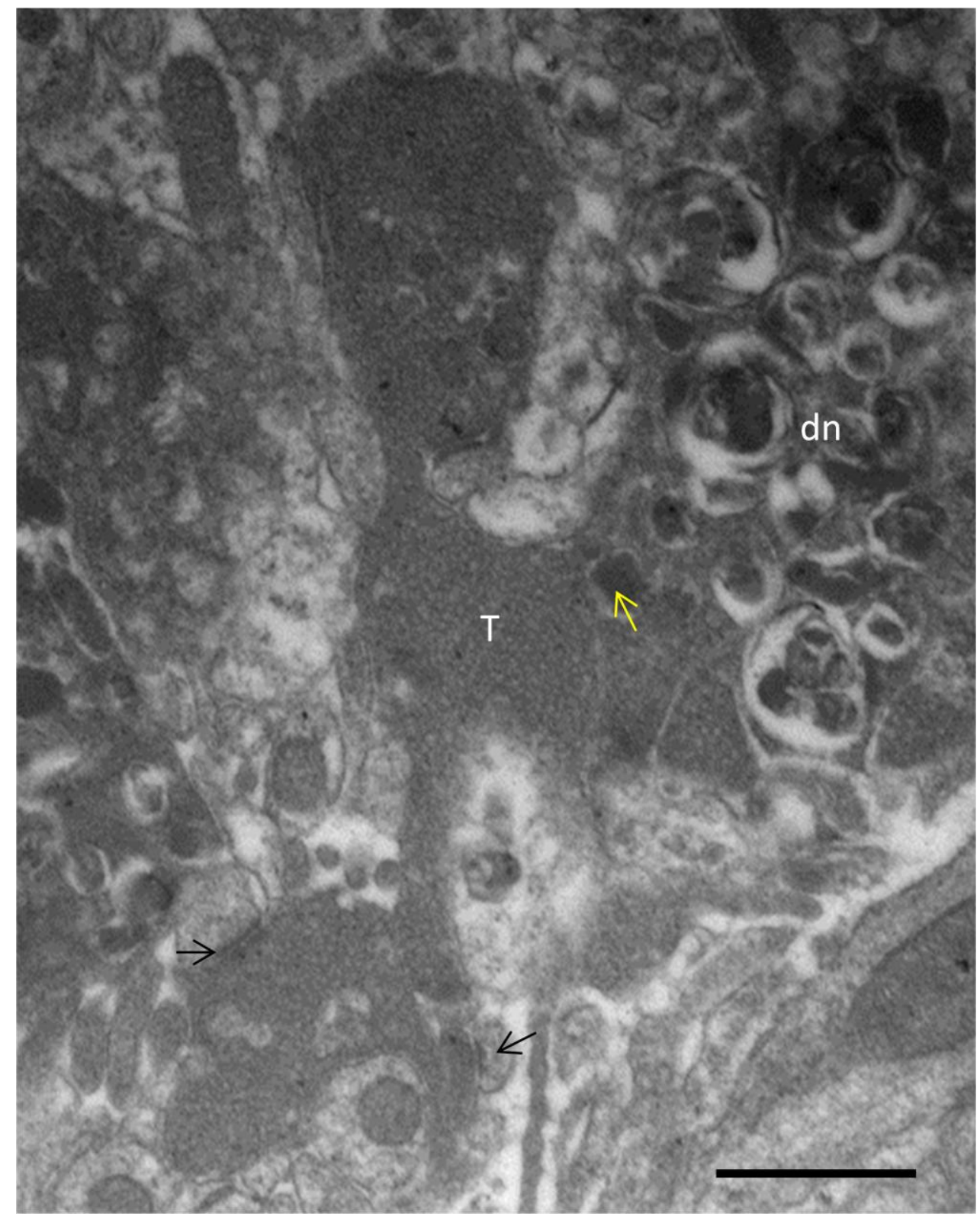


Figure 4.19 Intracellular amyloid core (red asterisk) inside the axon of 20-month old TgSwDI. Adjacent fibrillar bundles (black arrow) are possibly of amyloid origin; note the mitochondria (white arrow) and surrounding dystrophic neurites $(d n)$. Scale bar $=$ $2 \mu \mathrm{m}$ 


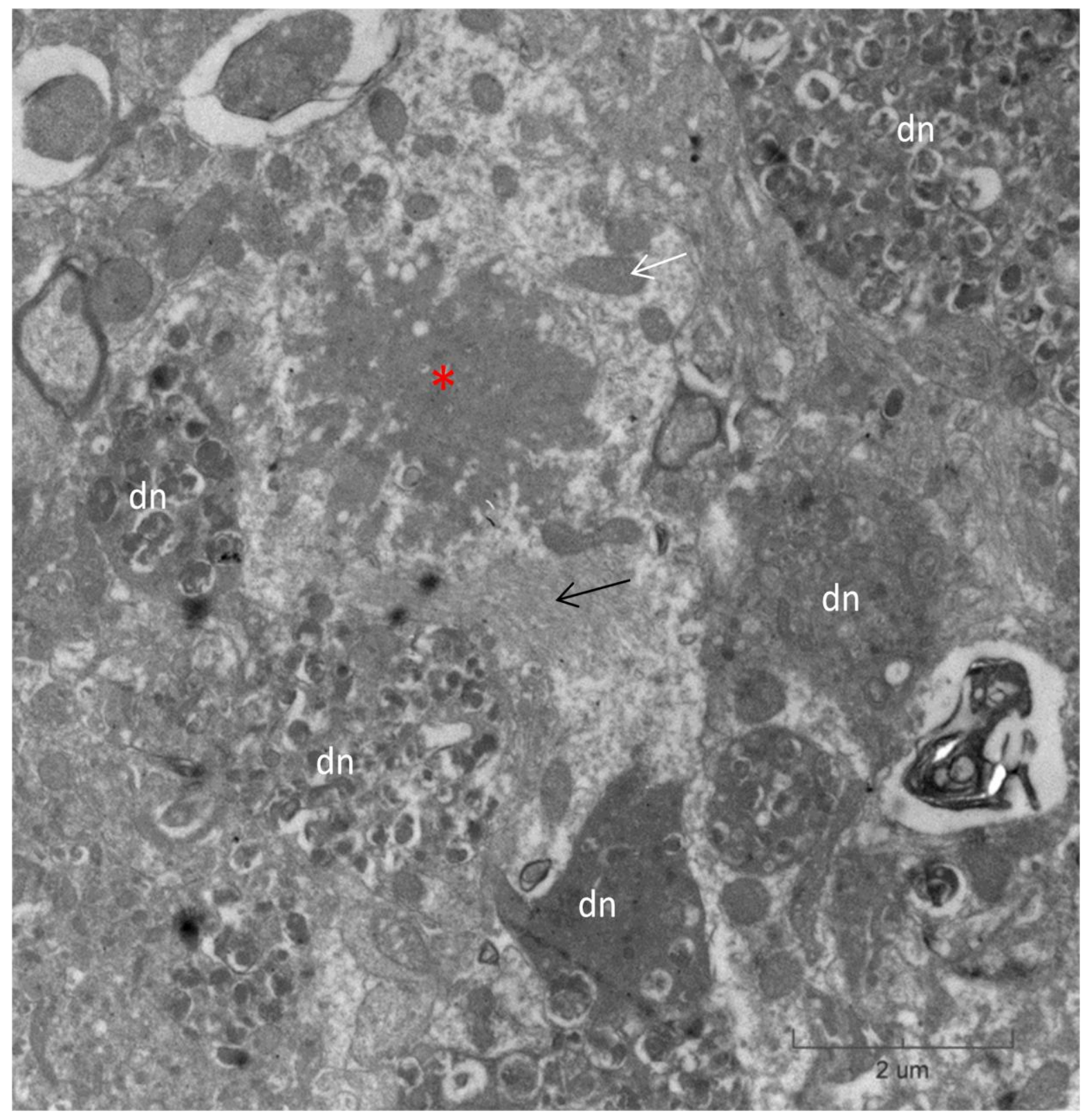


Figure 4.20 Fibrillar bundles in molecular layer of dentate gyrus in 13-month old TgSwDI. Fibrils lie among the mitochondria and resemble neurofibrillary tangles 


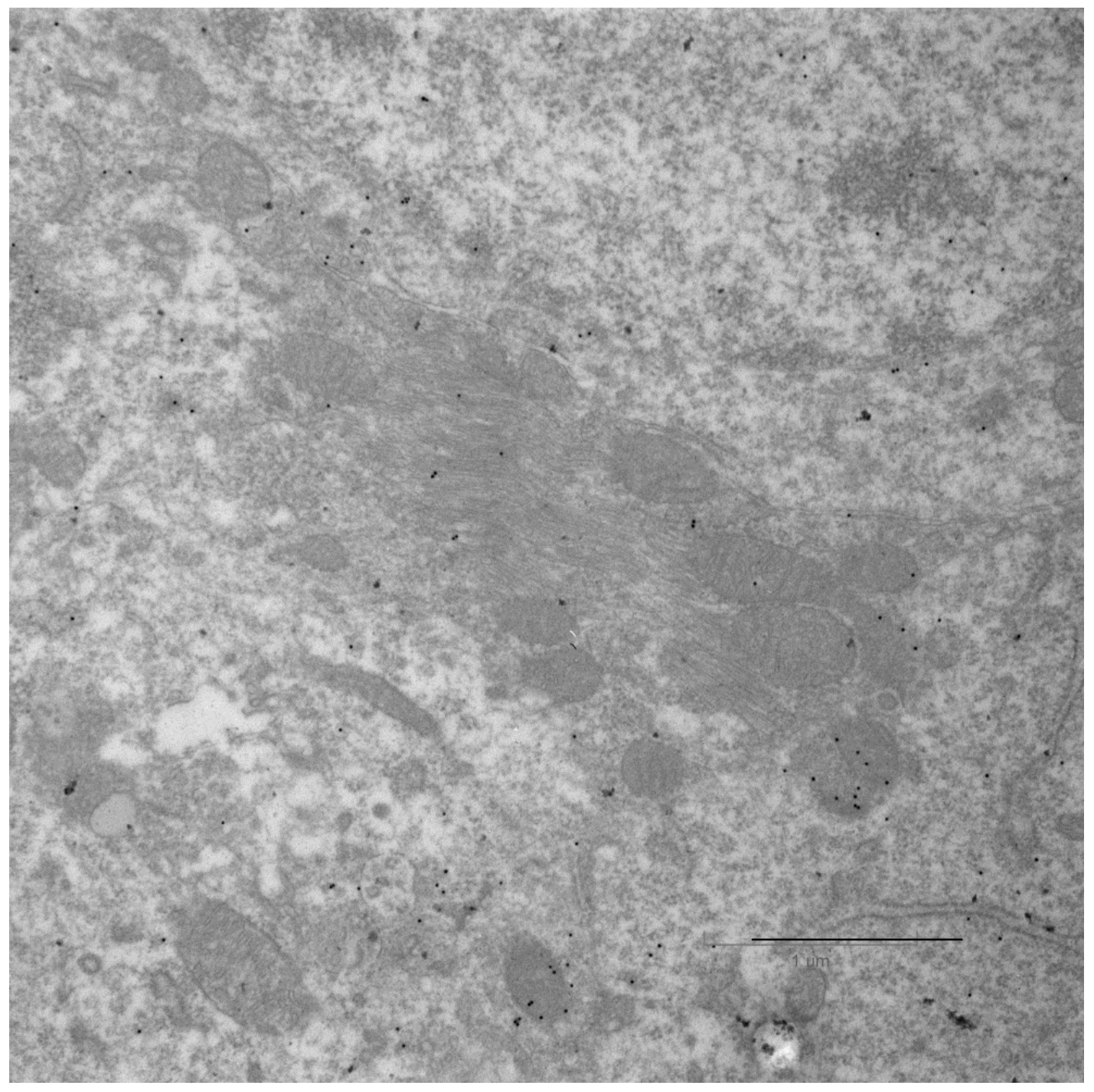


Figure 4.21 Microglial cell near dystrophic neurons showing signs of phagocytosis in 20-month old TgSwDI. Microglial cell (M) at periphery with lipofuscin (white arrow), vacuoles (yellow asterisk), inclusion bodies (black arrow) and vesicles

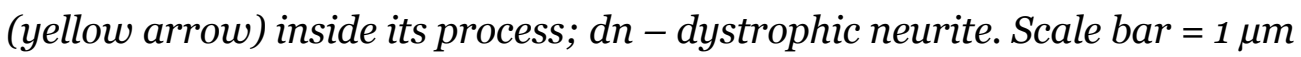




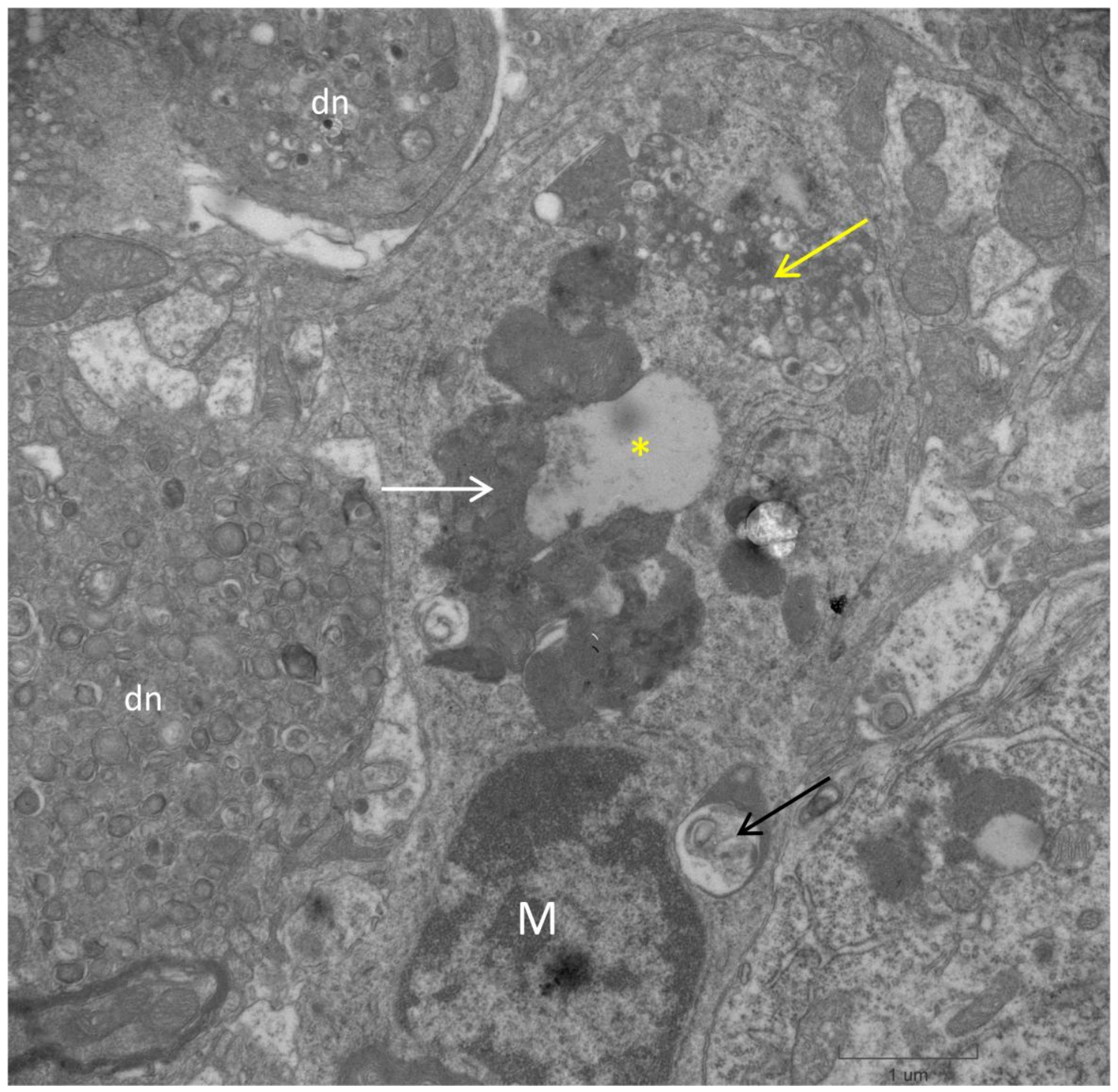




\section{CHAPTER V}

\section{SUMMARY, THEORETICAL CONSIDERATIONS AND FUTURE DIRECTIONS}

In this dissertation, which consists of several distinct chapters, the possible mechanisms of Alzheimer's disease (AD) development and histopathological characteristics of disease progression are investigated using a transgenic mouse model of AD. This model, with introduced triple Swedish/Dutch/Iowa mutations (TgSwDI), has a unique histopathological presentation manifesting in more pronounced cerebral amyloid angiopathy, which many consider to correlate with cognitive deterioration. Synaptic malfunction and loss, as the consequence of still not well understood etiologic factors of $\mathrm{AD}$, are also correlated with cognitive decline. The understanding of normal synaptic morphology and organization is therefore an important aspect of the continued search for the factors that affect synaptic malfunction and loss in AD.

In chapter II, we compared the synaptic organization of geniculocortical and pulvinocortical terminals in the tree shrew. We found several significant differences in the morphology of these terminals and their synaptic targets. Our results clearly demonstrate that the organization of thalamocortical synapses is quite different in the koniocortex and eulaminate cortex, although future studies will be necessary to determine the functional distinction between these projections. Future studies of other type of synapses in the koniocortex and eulaminate cortex may reveal features that would help to explain the different propensities of cortical regions to be affected by AD. In particular, $\mathrm{AD}$ pathology appears to primarily affect corticocortical cells. Therefore, future comparison of corticocortical connections may be a fruitful avenue to determine 
differences in cortical organization that may underlie the progression of AD pathology.

Chapter III investigated the potential interaction between zinc and spines as a possible pathophysiological mechanism of $\mathrm{AD}$ progression. Zinc, and the type 3 zinc transporter ( $\left.\mathrm{ZnT}_{3}\right)$, are thought to participate in different aspects of $\mathrm{AD}$ pathology. The earliest basis of memory deterioration is tied to the loss of dendritic spines, the most mature of which contain a unique organelle, the spine apparatus, which can be identified identified by the actin-binding protein synaptopodin. We measured changes in the levels of $\mathrm{ZnT}_{3}$ and synaptopodin in the cortex and hippocampus of the TgSwDI and WT mice at 1, 4 and 6 months of age before and after zinc precipitation via intraperitoneal injections of sodium selenite. Sodium selenite treatment produced no significant effect on the level of synaptopodin. However, we did find that $\mathrm{ZnT}_{3}$ levels were higher in TgSwDI at 4 months of age in both cortex and hippocampus when compared to wild type mice (WT) and TgSwDI mice of other ages. Also, when compared by ages, synaptopodin levels were higher in 4-month old WT and TgSwDI animals in both the cortex and hippocampus. These results suggest that zinc may be an important participant in the pathology of $\mathrm{AD}$, but that age-related changes in $\mathrm{ZnT} 3$ levels should be considered when evaluating treatments involving the manipulation of zinc levels.

Despite the seemingly strong hypothesis, we were unable to uncover any influence of zinc levels on the formation or maintenance of mature spines. It is worth noting, however, that the experiments were limited by a number of factors. 1) Few animals available so our experimental groups were small, 2) The sodium selenite injections proved to be quite toxic so were unable to test more long term effects of zinc precipitation, 3) we used animals at 1, 4 and 6 months of age but most of the pathological changes in the TgSwDI model used occur after 6 months of age and 4) the TgSwDI model we used does not produce the neuritic plaques typical of other AD models or the 
neuritic plaques and neurofibrillary tangles seen in human $\mathrm{AD}$ brains. Thus our results do not rule out a relationship between zinc and spines in $\mathrm{AD}$ pathology.

We conducted a power analysis to evaluate the possibility of our experiments reaching statistical significance if we increased the experimental groups described in Chapter III. To determine the probable sample sizes required to obtain significant differences, power analyses were performed on mean differences that were increased by $10 \%$ and with averaged standard deviations. Based on these power analyses, a significant difference would be expected in synaptopodin levels between the groups in the cortex at $1 \mathrm{hr}$ in 4 month old mice using a sample size of 6-7 per group with the power to predict this difference of $82.9-89.3 \%$, respectively. In the hippocampus a significant difference in synaptopodin may be achieved with sample sizes of 8-9 in 4 month olds at $1 \mathrm{hr}$. The power associated with these outcomes equaled 72.9 and $78.5 \%$, respectively. At 3 hrs the sample required increases to 15 per group and yields a power of $74.4 \%$ to detect a significant difference between the groups. Differences between the groups in $\mathrm{ZnT}_{3}$ in the cortex could be observed using sample sizes of 6 per group at 3 hrs in 4 month olds with a power of 93.0\%. The power to detect a significant difference in $\mathrm{ZnT}_{3}$ in the hippocampus equaled $86.0 \%$ in 1 month olds at $1 \mathrm{hr}$ with 7 per group and with 5-6 per group in 4 month olds at 3 hrs with a power of 97.0 and $99.1 \%$, respectively. Thus, further data collection would be necessary before drawing any final conclusions. Also, the experimental model could be changed by trying different zinc deprivation methods and different durations of zinc deprivation. The effect of increased zinc, for example via sensory deprivation, could also be used to assess the relationship between zinc and spine morphology.

Chapter IV described the histopathological features of the TgSwDI model used in our studies. We showed that isolectin b4 (Ib4) staining can be successfully used to study the age-related progression of AD pathology. Compared to humans and other mouse 
models of $\mathrm{AD}$, we found few classic neuritic plaques (with a dense $\mathrm{A} \beta$ core in the brain parenchyma). In the TgSwDI mice used in our studies, we instead found an abundant number of perivascular $A \beta$ depositions, which were surrounded by a corona of dystrophic neurites, microglial and astrocytic cells. Despite difference in the types of plaques found in different model, the timeline and sequential involvement of brain areas from enthorhinal/subiculum to the neocortex is similar. Overall, our results suggest that the TgSwDI mouse model is very suitable for the investigation of the cerebral amyloid angiopathy-related aspects of $\mathrm{AD}$.

The recent human trials of anti-A $\beta$ immunotherapy in advanced stages of $\mathrm{AD}$ pathology failed to produce significant cognitive improvement, although $A \beta$ was efficiently cleared from the brain. This raised new questions and led to the revised thoughts concerning the etipathogenetics of AD. Preventive measures and early intervention, before detrimental synaptic loss occurs, is the current priority of Alzheimer's research. The prevailing for almost two decades amyloid cascade hypothesis revealed large amount of weaknesses in its explanation of $\mathrm{AD}$ processes. Zinc dyshomeostasis was looked at as the igniting event that would precede amyloid cascade and could possibly explain the mechanism of amyloid formation as well as interference with other ultrastructural processes important for memory formation. The received data did not exclude importance of $\mathrm{A} \beta$ in the pathology of $\mathrm{AD}$ but it stressed the multifactorial nature of this disease. The existence of different oligomers of $A \beta$ and plaque forms described in Chapter IV also pointed out that there are disease types with pathology different from the one seen in typical $\mathrm{AD}$ postmortem brain, but yet exhibiting the similar cognitive dysfunction. These findings suggest that there are more pathogenetic pathways are involved in $\mathrm{AD}$ than just amyloid cascade. The function of microglia in $\mathrm{AD}$ is debated intensely with some authors ascribing these cells a major role in the aggravation of pathology while others demonstrating that $\mathrm{AD}$ pathology can develop 
without microglial participation. In either case, further studies are needed to understand every aspect of this disease. If we understand why pathology starts in certain areas of the brain, this could lead to methods to prevent the initial onset of AD. Finding ways to direct the balance between the necessary phagocytic properties of microglia and the detrimental properties of excessive microglial influx is another priority. Finally, investigating what role cerebral amyloid angiopathy plays in the progression of $\mathrm{AD}$ is another promising direction in $\mathrm{AD}$ research.

Despite the enormous number of patients affected by $\mathrm{AD}$ pathology and the multitude of hypotheses regarding the causes and treatment of this detrimental disease, we are still far from the desperately needed answers. The hope for a cure still exists while the minds of passionate investigators remain eager to solve one of the most complicated mysteries in the field of medicine to date. 


\section{REFERENCES}

1. $\quad$ Abbott, R.D., et al., Walking and dementia in physically capable elderly men. JAMA : the journal of the American Medical Association, 2004. 292(12): p. 144753.

2. Acsady, L., et al., GABAergic cells are the major postsynaptic targets of mossy fibers in the rat hippocampus. The Journal of neuroscience : the official journal of the Society for Neuroscience, 1998. 18(9): p. 3386-403.

3. $\quad$ Adlard, P.A., et al., Metal ionophore treatment restores dendritic spine density and synaptic protein levels in a mouse model of Alzheimer's disease. PloS one, 2011. 6(3): p. e17669.

4. Adlard, P.A. and A.I. Bush, Metals and Alzheimer's disease. Journal of Alzheimer's disease : JAD, 2006. 10(2-3): p. 145-63.

5. $\quad$ Adlard, P.A., et al., Rapid restoration of cognition in Alzheimer's transgenic mice with 8-hydroxy quinoline analogs is associated with decreased interstitial Abeta. Neuron, 2008. 59(1): p. 43-55.

6. Adlard, P.A., et al., Cognitive loss in zinc transporter-3 knock-out mice: a phenocopy for the synaptic and memory deficits of Alzheimer's disease? The Journal of neuroscience : the official journal of the Society for Neuroscience, 2010. 30(5): p. 1631-6.

7. Aguzzi, A., B.A. Barres, and M.L. Bennett, Microglia: scapegoat, saboteur, or something else? Science, 2013. 339(6116): p. 156-61.

8. Ambree, O., et al., Reduction of amyloid angiopathy and Abeta plaque burden after enriched housing in TgCRND8 mice: involvement of multiple pathways. The American journal of pathology, 2006. 169(2): p. 544-52.

9. Andersen, P., T.V. Bliss, and K.K. Skrede, Lamellar organization of hippocampal pathways. Experimental brain research. Experimentelle Hirnforschung. Experimentation cerebrale, 1971. 13(2): p. 222-38.

10. Anderson, J.C. and K.A. Martin, Connection from cortical area V2 to MT in macaque monkey. The Journal of comparative neurology, 2002. 443(1): p. 5670.

11. Andra, K., et al., Expression of APP in transgenic mice: a comparison of neuronspecific promoters. Neurobiology of aging, 1996. 17(2): p. 183-90.

12. Applegate, M.D. and P.W. Landfield, Synaptic vesicle redistribution during hippocampal frequency potentiation and depression in young and aged rats. The Journal of neuroscience : the official journal of the Society for Neuroscience, 1988. 8(4): p. 1096-111.

13. Arendash, G.W., et al., Environmental enrichment improves cognition in aged Alzheimer's transgenic mice despite stable beta-amyloid deposition. Neuroreport, 2004. 15(11): p. 1751-4.

14. Armstrong, R.A., N.J. Cairns, and P.L. Lantos, beta-Amyloid (A beta) deposition in the medial temporal lobe of patients with dementia with Lewy bodies. Neuroscience letters, 1997. 227(3): p. 193-6.

15. Arriagada, P.V., K. Marzloff, and B.T. Hyman, Distribution of Alzheimer-type pathologic changes in nondemented elderly individuals matches the pattern in Alzheimer's disease. Neurology, 1992. 42(9): p. 1681-8. 
16. Ascoli, G.A., et al., Petilla terminology: nomenclature of features of GABAergic interneurons of the cerebral cortex. Nature reviews. Neuroscience, 2008. 9(7): p. 557-68.

17. Ashby, M.C. and J.T. Isaac, Maturation of a recurrent excitatory neocortical circuit by experience-dependent unsilencing of newly formed dendritic spines. Neuron, 2011. 70(3): p. 510-21.

18. Ashby, M.C., et al., Lateral diffusion drives constitutive exchange of AMPA receptors at dendritic spines and is regulated by spine morphology. The Journal of neuroscience : the official journal of the Society for Neuroscience, 2006. 26(26): p. 7046-55.

19. Avila, J., Tau protein, the main component of paired helical filaments. Journal of Alzheimer's disease : JAD, 2006. 9(3 Suppl): p. 171-5.

20. Bas Orth, C., et al., Loss of the cisternal organelle in the axon initial segment of cortical neurons in synaptopodin-deficient mice. The Journal of comparative neurology, 2007. 504(5): p. 441-9.

21. Baumketner, A., M.G. Krone, and J.E. Shea, Role of the familial Dutch mutation E22Q in the folding and aggregation of the 15-28 fragment of the Alzheimer amyloid-beta protein. Proceedings of the National Academy of Sciences of the United States of America, 2008. 105(16): p. 6027-32.

22. Bayer, T.A. and O. Wirths, Intracellular accumulation of amyloid-Beta - a predictor for synaptic dysfunction and neuron loss in Alzheimer's disease. Frontiers in aging neuroscience, 2010. 2: p. 8.

23. Bender, D.B. and C.M. Butter, Comparison of the effects of superior colliculus and pulvinar lesions on visual search and tachistoscopic pattern discrimination in monkeys. Experimental brain research. Experimentelle Hirnforschung.

Experimentation cerebrale, 1987. 69(1): p. 140-54.

24. Bennett, D.A., Postmortem indices linking risk factors to cognition: results from the Religious Order Study and the Memory and Aging Project. Alzheimer disease and associated disorders, 2006. 2o(3 Suppl 2): p. S63-8.

25. Besser, L., et al., Synaptically released zinc triggers metabotropic signaling via a zinc-sensing receptor in the hippocampus. The Journal of neuroscience : the official journal of the Society for Neuroscience, 2009. 29(9): p. 2890-901.

26. Bettens, K., K. Sleegers, and C. Van Broeckhoven, Genetic insights in Alzheimer's disease. Lancet neurology, 2013. 12(1): p. 92-104.

27. Bibl, M., et al., CSF amyloid-beta-peptides in Alzheimer's disease, dementia with Lewy bodies and Parkinson's disease dementia. Brain : a journal of neurology, 2006. 129(Pt 5): p. 1177-87.

28. Binzegger, T., R.J. Douglas, and K.A. Martin, A quantitative map of the circuit of cat primary visual cortex. The Journal of neuroscience : the official journal of the Society for Neuroscience, 2004. 24(39): p. 8441-53.

29. Blennow, K., et al., Effect of immunotherapy with bapineuzumab on cerebrospinal fluid biomarker levels in patients with mild to moderate Alzheimer disease. Archives of neurology, 2012. 69(8): p. 1002-10.

30. Bourne, J. and K.M. Harris, Do thin spines learn to be mushroom spines that remember? Current opinion in neurobiology, 2007. 17(3): p. 381-6.

31. Braak, H., et al., Staging of Alzheimer disease-associated neurofibrillary pathology using paraffin sections and immunocytochemistry. Acta neuropathologica, 2006. 112(4): p. 389-404.

32. Braak, H. and E. Braak, Neuropathological stageing of Alzheimer-related changes. Acta neuropathologica, 1991. 82(4): p. 239-59. 
33. Braak, H., E. Braak, and J. Bohl, Staging of Alzheimer-related cortical destruction. European neurology, 1993. 33(6): p. 403-8.

34. Brookmeyer, R., S. Gray, and C. Kawas, Projections of Alzheimer's disease in the United States and the public health impact of delaying disease onset. American journal of public health, 1998. 88(9): p. 1337-42.

35. Brown, C.E. and R.H. Dyck, Rapid, experience-dependent changes in levels of synaptic zinc in primary somatosensory cortex of the adult mouse. The Journal of neuroscience : the official journal of the Society for Neuroscience, 2002. 22(7): p. 2617-25.

36. Brown, C.E. and R.H. Dyck, Retrograde tracing of the subset of afferent connections in mouse barrel cortex provided by zincergic neurons. The Journal of comparative neurology, 2005. 486(1): p. 48-60.

37. Brown, S.P. and S. Hestrin, Cell-type identity: a key to unlocking the function of neocortical circuits. Current opinion in neurobiology, 2009. 19(4): p. 415-21.

38. Buhl, E.H. and W. Singer, The callosal projection in cat visual cortex as revealed by a combination of retrograde tracing and intracellular injection.

Experimental brain research. Experimentelle Hirnforschung. Experimentation cerebrale, 1989. 75(3): p. 470-6.

39. Buki, A. and J.T. Povlishock, All roads lead to disconnection?--Traumatic axonal injury revisited. Acta neurochirurgica, 2006. 148(2): p. 181-93; discussion 193-4.

40. Bush, A.I., et al., Rapid induction of Alzheimer $A$ beta amyloid formation by zinc. Science, 1994. 265(5177): p. 1464-7.

41. Bush, A.I. and R.E. Tanzi, Therapeutics for Alzheimer's disease based on the metal hypothesis. Neurotherapeutics : the journal of the American Society for Experimental NeuroTherapeutics, 2008. 5(3): p. 421-32.

42. Capasso, M., et al., Zinc dyshomeostasis: a key modulator of neuronal injury. Journal of Alzheimer's disease : JAD, 2005. 8(2): p. 93-108; discussion 209-15.

43. Carder, R.K. and S.H. Hendry, Neuronal characterization, compartmental distribution, and activity-dependent regulation of glutamate immunoreactivity in adult monkey striate cortex. The Journal of neuroscience : the official journal of the Society for Neuroscience, 1994. 14(1): p. 242-62.

44. Catala, I., et al., Decreased numbers of dendritic spines on cortical pyramidal neurons in dementia. A quantitative Golgi study on biopsy samples. Human neurobiology, 1988. 6(4): p. 255-9.

45. Chen, H. and J.H. Zhang, Cerebral amyloid angiopathy-related microhemorrhages in Alzheimer's disease: a review of investigative animal models. Acta neurochirurgica. Supplement, 2011. 111: p. 15-7.

46. Chen, H.S. and S.A. Lipton, Mechanism of memantine block of NMDA-activated channels in rat retinal ganglion cells: uncompetitive antagonism. The Journal of physiology, 1997. 499 ( Pt 1): p. 27-46.

47. Chen, H.S. and S.A. Lipton, The chemical biology of clinically tolerated NMDA receptor antagonists. Journal of neurochemistry, 2006. 97(6): p. 1611-26.

48. Chomsung, R.D., H.M. Petry, and M.E. Bickford, Ultrastructural examination of diffuse and specific tectopulvinar projections in the tree shrew. The Journal of comparative neurology, 2008. 510(1): p. 24-46.

49. Chomsung, R.D., et al., Synaptic organization of connections between the temporal cortex and pulvinar nucleus of the tree shrew. Cerebral cortex, 2010. 20(4): p. 997-1011.

50. Chung, H., et al., Uptake, degradation, and release of fibrillar and soluble forms of Alzheimer's amyloid beta-peptide by microglial cells. The Journal of biological chemistry, 1999. 274(45): p. 32301-8. 
51. Claiborne, B.J., D.G. Amaral, and W.M. Cowan, A light and electron microscopic analysis of the mossy fibers of the rat dentate gyrus. The Journal of comparative neurology, 1986. 246(4): p. 435-58.

52. Cole, T.B., et al., Elimination of zinc from synaptic vesicles in the intact mouse brain by disruption of the ZnT3 gene. Proceedings of the National Academy of Sciences of the United States of America, 1999. 96(4): p. 1716-21.

53. Coleman, J.E., et al., Rapid structural remodeling of thalamocortical synapses parallels experience-dependent functional plasticity in mouse primary visual cortex. The Journal of neuroscience : the official journal of the Society for Neuroscience, 2010. 30(29): p. 9670-82.

54. Collins, C.E., et al., Optical imaging of visually evoked responses in the middle temporal area after deactivation of primary visual cortex in adult primates. Proceedings of the National Academy of Sciences of the United States of America, 2005. 102(15): p. 5594-9.

55. Collins, J., et al., Modality Switching in a Property Verification Task: An ERP Study of What Happens When Candles Flicker after High Heels Click. Frontiers in psychology, 2011. 2: p. 10.

56. Conley, M., D. Fitzpatrick, and I.T. Diamond, The laminar organization of the lateral geniculate body and the striate cortex in the tree shrew (Tupaia glis). The Journal of neuroscience : the official journal of the Society for Neuroscience, 1984. 4(1): p. 171-97.

57. Corson, J., et al., Sensory activity differentially modulates N-methyl-Daspartate receptor subunits $2 A$ and $2 B$ in cortical layers. Neuroscience, 2009. 163(3): p. 920-32.

58. Costa, D.A., et al., Enrichment improves cognition in AD mice by amyloidrelated and unrelated mechanisms. Neurobiology of aging, 2007. 28(6): p. 83144 .

59. $\quad$ Cotel, M.C., et al., Environmental enrichment fails to rescue working memory deficits, neuron loss, and neurogenesis in APP/PS1KI mice. Neurobiology of aging, 2012. 33(1): p. 96-107.

6o. Cousins, R.J., J.P. Liuzzi, and L.A. Lichten, Mammalian zinc transport, trafficking, and signals. The Journal of biological chemistry, 2006. 281(34): p. 24085-9.

61. Cowey, A. and P. Stoerig, Visual detection in monkeys with blindsight. Neuropsychologia, 1997. 35(7): p. 929-39.

62. Cracchiolo, J.R., et al., Enhanced cognitive activity--over and above social or physical activity--is required to protect Alzheimer's mice against cognitive impairment, reduce Abeta deposition, and increase synaptic immunoreactivity. Neurobiology of learning and memory, 2007. 88(3): p. 277-94.

63. Crimins, J.L., et al., The intersection of amyloid beta and tau in glutamatergic synaptic dysfunction and collapse in Alzheimer's disease. Ageing research reviews, 2013.

64. da Costa, N.M. and K.A. Martin, How thalamus connects to spiny stellate cells in the cat's visual cortex. The Journal of neuroscience : the official journal of the Society for Neuroscience, 2011. 31(8): p. 2925-37.

65. Danckert, J. and Y. Rossetti, Blindsight in action: what can the different subtypes of blindsight tell us about the control of visually guided actions?

Neuroscience and biobehavioral reviews, 2005. 29(7): p. 1035-46.

66. D'Andrea, M.R., G.M. Cole, and M.D. Ard, The microglial phagocytic role with specific plaque types in the Alzheimer disease brain. Neurobiology of aging, 2004. 25(5): p. 675-83. 
67. Danscher, G. and M. Stoltenberg, Silver enhancement of quantum dots resulting from (1) metabolism of toxic metals in animals and humans, (2) in vivo, in vitro and immersion created zinc-sulphur/zinc-selenium nanocrystals, (3) metal ions liberated from metal implants and particles. Progress in histochemistry and cytochemistry, 2006. 41(2): p. 57-139.

68. Davies, C.A., et al., A quantitative morphometric analysis of the neuronal and synaptic content of the frontal and temporal cortex in patients with Alzheimer's disease. Journal of the neurological sciences, 1987. 78(2): p. 151-64.

69. Davis, J. and W.E. Van Nostrand, Enhanced pathologic properties of Dutch-type mutant amyloid beta-protein. Proceedings of the National Academy of Sciences of the United States of America, 1996. 93(7): p. 2996-3000.

70. Davis, J., et al., Early-onset and robust cerebral microvascular accumulation of amyloid beta-protein in transgenic mice expressing low levels of a vasculotropic Dutch/Iowa mutant form of amyloid beta-protein precursor. The Journal of biological chemistry, 2004. 279(19): p. 20296-306.

71. Davis, J., et al., Deficient cerebral clearance of vasculotropic mutant Dutch/Iowa Double A beta in human A betaPP transgenic mice. Neurobiology of aging, 2006. 27(7): p. 946-54.

72. Day-Brown, J.D., et al., Pulvinar projections to the striatum and amygdala in the tree shrew. Frontiers in neuroanatomy, 2010. 4: p. 143.

73. de Ruiter, J.P. and H.B. Uylings, Morphometric and dendritic analysis of fascia dentata granule cells in human aging and senile dementia. Brain research, 1987. 402(2): p. 217-29.

74. Deane, R., Z. Wu, and B.V. Zlokovic, RAGE (yin) versus LRP (yang) balance regulates alzheimer amyloid beta-peptide clearance through transport across the blood-brain barrier. Stroke; a journal of cerebral circulation, 2004. 35(11 Suppl 1): p. 2628-31.

75. Dehay, C., et al., Excitation by geniculocortical synapses is not 'vetoed' at the level of dendritic spines in cat visual cortex. The Journal of physiology, 1991. 440: p. 723-34.

76. DeKosky, S.T. and S.W. Scheff, Synapse loss in frontal cortex biopsies in Alzheimer's disease: correlation with cognitive severity. Annals of neurology, 1990. 27(5): p. 457-64.

77. DeKosky, S.T., S.W. Scheff, and S.D. Styren, Structural correlates of cognition in dementia: quantification and assessment of synapse change.

Neurodegeneration : a journal for neurodegenerative disorders, neuroprotection, and neuroregeneration, 1996. 5(4): p. 417-21.

78. Deller, T., et al., A role for synaptopodin and the spine apparatus in hippocampal synaptic plasticity. Annals of anatomy = Anatomischer Anzeiger : official organ of the Anatomische Gesellschaft, 2007. 189(1): p. 5-16.

79. Deller, T., et al., Synaptopodin-deficient mice lack a spine apparatus and show deficits in synaptic plasticity. Proceedings of the National Academy of Sciences of the United States of America, 2003. 100(18): p. 10494-9.

8o. Deshpande, A., et al., A role for synaptic zinc in activity-dependent Abeta oligomer formation and accumulation at excitatory synapses. The Journal of neuroscience : the official journal of the Society for Neuroscience, 2009. 29(13): p. 4004-15.

81. Dikranian, K., et al., Ultrastructural studies in APP/PS1 mice expressing human ApoE isoforms: implications for Alzheimer's disease. International journal of clinical and experimental pathology, 2012. 5(6): p. 482-95. 
82. Doering, P., et al., Chemical blocking of zinc ions in CNS increases neuronal damage following traumatic brain injury (TBI) in mice. PloS one, 2010. 5(4): p. e10131.

83. Dukes, K.D., C.F. Rodenberg, and R.K. Lammi, Monitoring the earliest amyloidbeta oligomers via quantized photobleaching of dye-labeled peptides. Analytical biochemistry, 2008. 382(1): p. 29-34.

84. Dyck, R.H., A. Chaudhuri, and M.S. Cynader, Experience-dependent regulation of the zincergic innervation of visual cortex in adult monkeys. Cerebral cortex, 2003. 13(10): p. 1094-109.

85. Einstein, G. and D. Fitzpatrick, Distribution and morphology of area 17 neurons that project to the cat's extrastriate cortex. The Journal of comparative neurology, 1991. 303(1): p. 132-49.

86. Erisir, A. and M. Dreusicke, Quantitative morphology and postsynaptic targets of thalamocortical axons in critical period and adult ferret visual cortex. The Journal of comparative neurology, 2005. 485(1): p. 11-31.

87. Evans, D.A., et al., Prevalence of Alzheimer's disease in a community population of older persons. Higher than previously reported. JAMA : the journal of the American Medical Association, 1989. 262(18): p. 2551-6.

88. Ewbank, D.C. and S.E. Arnold, Cool with plaques and tangles. The New England journal of medicine, 2009. 360(22): p. 2357-9.

89. Farlow, M., et al., Safety and biomarker effects of solanezumab in patients with Alzheimer's disease. Alzheimer's \& dementia : the journal of the Alzheimer's Association, 2012. 8(4): p. 261-71.

90. Faux, N.G., et al., PBT2 rapidly improves cognition in Alzheimer's Disease: additional phase II analyses. Journal of Alzheimer's disease : JAD, 2010. 20(2): p. 509-16.

91. Feldman, S.C. and A.J. Kastin, Localization of neurons containing immunoreactive delta sleep-inducing peptide in the rat brain: an immunocytochemical study. Neuroscience, 1984. 11(2): p. 303-17.

92. Fendrick, S.E., Q.S. Xue, and W.J. Streit, Formation of multinucleated giant cells and microglial degeneration in rats expressing a mutant $\mathrm{Cu} / \mathrm{Zn}$ superoxide dismutase gene. Journal of neuroinflammation, 2007. 4: p. 9.

93. Fiala, J.C., et al., Synaptogenesis via dendritic filopodia in developing hippocampal area CA1. The Journal of neuroscience : the official journal of the Society for Neuroscience, 1998. 18(21): p. 8900-11.

94. Fitzpatrick, D., The functional organization of local circuits in visual cortex: insights from the study of tree shrew striate cortex. Cerebral cortex, 1996. 6(3): p. 329-41.

95. Fitzpatrick, D., R.G. Carey, and I.T. Diamond, The projection of the superior colliculus upon the lateral geniculate body in Tupaia glis and Galago senegalensis. Brain research, 1980. 194(2): p. 494-9.

96. Frackowiak, J., et al., Ultrastructure of the microglia that phagocytose amyloid and the microglia that produce beta-amyloid fibrils. Acta neuropathologica, 1992. 84(3): p. 225-33.

97. Frazzini, V., et al., Oxidative stress and brain aging: is zinc the link? Biogerontology, 2006. 7(5-6): p. 307-14.

98. Frederickson, C.J., J.Y. Koh, and A.I. Bush, The neurobiology of zinc in health and disease. Nature reviews. Neuroscience, 2005. 6(6): p. 449-62.

99. Frederickson, C.J., et al., Importance of zinc in the central nervous system: the zinc-containing neuron. The Journal of nutrition, 2000. 130(5S Suppl): p. $1471 \mathrm{~S}-83 \mathrm{~S}$. 
100. Freund, T.F., et al., Arborisation pattern and postsynaptic targets of physiologically identified thalamocortical afferents in striate cortex of the macaque monkey. The Journal of comparative neurology, 1989. 289(2): p. 31536.

101. Friedlich, A.L., et al., Neuronal zinc exchange with the blood vessel wall promotes cerebral amyloid angiopathy in an animal model of Alzheimer's disease. The Journal of neuroscience : the official journal of the Society for Neuroscience, 2004. 24(13): p. 3453-9.

102. Gage, F.H. and K.S. Chen, Neural transplants: prospects for Alzheimer's disease. Current opinion in neurology and neurosurgery, 1992. 5(1): p. 94-9.

103. Gaither, L.A. and D.J. Eide, The human ZIP1 transporter mediates zinc uptake in human K562 erythroleukemia cells. The Journal of biological chemistry, 2001. 276(25): p. 22258-64.

104. Galasso, S.L. and R.H. Dyck, The role of zinc in cerebral ischemia. Molecular medicine, 2007. 13(7-8): p. 380-7.

105. Ganeshina, O., et al., Synapses with a segmented, completely partitioned postsynaptic density express more AMPA receptors than other axospinous synaptic junctions. Neuroscience, 2004. 125(3): p. 615-23.

106. Garai, K., et al., Selective destabilization of soluble amyloid beta oligomers by divalent metal ions. Biochemical and biophysical research communications, 2006. 345(1): p. 210-5.

107. Garey, L.J. and T.P. Powell, An experimental study of the termination of the lateral geniculo-cortical pathway in the cat and monkey. Proceedings of the Royal Society of London. Series B, Containing papers of a Biological character. Royal Society, 1971. 179(54): p. 41-63.

108. Gaudet, A.D., P.G. Popovich, and M.S. Ramer, Wallerian degeneration: gaining perspective on inflammatory events after peripheral nerve injury. Journal of neuroinflammation, 2011. 8: p. 110.

109. Gentleman, S.M., et al., A demonstration of the advantages of immunostaining in the quantification of amyloid plaque deposits. Histochemistry, 1989. 92(4): p. 355-8.

110. Gilbert, C.D. and T.N. Wiesel, Morphology and intracortical projections of functionally characterised neurones in the cat visual cortex. Nature, 1979. 280(5718): p. 120-5.

111. Goodale, M.A. and A.D. Milner, Separate visual pathways for perception and action. Trends in neurosciences, 1992. 15(1): p. 20-5.

112. Grabowski, T.J., et al., Novel amyloid precursor protein mutation in an Iowa family with dementia and severe cerebral amyloid angiopathy. Annals of neurology, 2001. 49(6): p. 697-705.

113. Griffin, W.S., et al., Brain interleukin 1 and $S$-10o immunoreactivity are elevated in Down syndrome and Alzheimer disease. Proceedings of the National Academy of Sciences of the United States of America, 1989. 86(19): p. 7611-5.

114. Ha, C., J. Ryu, and C.B. Park, Metal ions differentially influence the aggregation and deposition of Alzheimer's beta-amyloid on a solid template. Biochemistry, 2007. 46(20): p. 6118-25.

115. Hardy, J. and D. Allsop, Amyloid deposition as the central event in the aetiology of Alzheimer's disease. Trends in pharmacological sciences, 1991. 12(10): p. 3838.

116. Hardy, J.A. and G.A. Higgins, Alzheimer's disease: the amyloid cascade hypothesis. Science, 1992. 256(5054): p. 184-5. 
117. Harms, K.J. and A. Dunaevsky, Dendritic spine plasticity: looking beyond development. Brain research, 2007. 1184: p. 65-71.

118. Haroutunian, V., et al., Role of the neuropathology of Alzheimer disease in dementia in the oldest-old. Archives of neurology, 2008. 65(9): p. 1211-7.

119. Harris, K.M. and J.K. Stevens, Dendritic spines of CA 1 pyramidal cells in the rat hippocampus: serial electron microscopy with reference to their biophysical characteristics. The Journal of neuroscience : the official journal of the Society for Neuroscience, 1989. 9(8): p. 2982-97.

120. Harvey, C.D. and K. Svoboda, Locally dynamic synaptic learning rules in pyramidal neuron dendrites. Nature, 2007. 450(7173): p. 1195-200.

121. Helzner, E.P., et al., Leisure activity and cognitive decline in incident Alzheimer disease. Archives of neurology, 2007. 64(12): p. 1749-54.

122. Heneka, M.T., et al., Focal glial activation coincides with increased BACE1 activation and precedes amyloid plaque deposition in APP [V717I] transgenic mice. Journal of neuroinflammation, 2005. 2: p. 22.

123. Henze, D.A., N.N. Urban, and G. Barrionuevo, The multifarious hippocampal mossy fiber pathway: a review. Neuroscience, 2000. 98(3): p. 407-27.

124. Herzig, M.C., et al., Abeta is targeted to the vasculature in a mouse model of hereditary cerebral hemorrhage with amyloidosis. Nature neuroscience, 2004. 7(9): p. 954-6o.

125. Hirsch, J.A., et al., Functionally distinct inhibitory neurons at the first stage of visual cortical processing. Nature neuroscience, 2003. 6(12): p. 1300-8.

126. Holtmaat, A.J., et al., Transient and persistent dendritic spines in the neocortex in vivo. Neuron, 2005. 45(2): p. 279-91.

127. Hosmane, S., et al., Toll/interleukin-1 receptor domain-containing adapter inducing interferon-beta mediates microglial phagocytosis of degenerating axons. The Journal of neuroscience : the official journal of the Society for Neuroscience, 2012. 32(22): p. 7745-57.

128. Hsiao, K., Transgenic mice expressing Alzheimer amyloid precursor proteins. Experimental gerontology, 1998. 33(7-8): p. 883-9.

129. Huang, W.C., et al., TGF-beta1 blockade of microglial chemotaxis toward Abeta aggregates involves SMAD signaling and down-regulation of CCL5. Journal of neuroinflammation, 2010. 7: p. 28.

130. Huang, Y., et al., Apolipoprotein E fragments present in Alzheimer's disease brains induce neurofibrillary tangle-like intracellular inclusions in neurons. Proceedings of the National Academy of Sciences of the United States of America, 2001. 98(15): p. 8838-43.

131. Huang, Y.Z., et al., Zinc-mediated transactivation of TrkB potentiates the hippocampal mossy fiber-CA3 pyramid synapse. Neuron, 2008. 57(4): p. 54658.

132. Hubener, M., C. Schwarz, and J. Bolz, Morphological types of projection neurons in layer 5 of cat visual cortex. The Journal of comparative neurology, 1990. 301(4): p. 655-74.

133. Iacono, D., et al., The Nun study: clinically silent $A D$, neuronal hypertrophy, and linguistic skills in early life. Neurology, 2009. 73(9): p. 665-73.

134. Irizarry, M.C., et al., APPSw transgenic mice develop age-related A beta deposits and neuropil abnormalities, but no neuronal loss in CA1. Journal of neuropathology and experimental neurology, 1997. 56(9): p. 965-73.

135. Itoh, K., M. Conley, and I.T. Diamond, Different distribution of large and small retinal ganglion cells in the cat after $H R P$ injections of single layers of the 
lateral geniculate body and the superior colliculus. Brain research, 1981. 207(1): p. 147-52.

136. Itoh, K., M. Conley, and I.T. Diamond, Retinal ganglion cell projections to individual layers of the lateral geniculate body in Galago crassicaudatus. The Journal of comparative neurology, 1982. 205(3): p. 282-90.

137. Jedlicka, P., et al., Impairment of in vivo theta-burst long-term potentiation and network excitability in the dentate gyrus of synaptopodin-deficient mice lacking the spine apparatus and the cisternal organelle. Hippocampus, 2009. 19(2): p. 130-40.

138. Jedlicka, P., et al., A role for the spine apparatus in LTP and spatial learning. Behavioural brain research, 2008. 192(1): p. 12-9.

139. Jellinger, K.A., Pathology and pathophysiology of vascular cognitive impairment. A critical update. Panminerva medica, 2004. 46(4): p. 217-26.

140. Jellinger, K.A., Alzheimer's disease: a challenge for modern neuropathobiology. Acta neuropathologica, 2009. 118(1): p. 1-3.

141. Jendroska, K., et al., Amyloid beta-peptide and its relationship with dementia in Lewy body disease. Journal of neural transmission. Supplementum, 1997. 51: p. 137-44.

142. Jendroska, K., et al., Amyloid beta-peptide and the dementia of Parkinson's disease. Movement disorders : official journal of the Movement Disorder Society, 1996. 11(6): p. 647-53.

143. Jones, E.G. and T.P. Powell, Morphological variations in the dendritic spines of the neocortex. Journal of cell science, 1969. 5(2): p. 509-29.

144. Kang, J., et al., The precursor of Alzheimer's disease amyloid A4 protein resembles a cell-surface receptor. Nature, 1987. 325(6106): p. 733-6.

145. Kasai, H., et al., Structural dynamics of dendritic spines in memory and cognition. Trends in neurosciences, 2010. 33(3): p. 121-9.

146. Katz, L.C., Local circuitry of identified projection neurons in cat visual cortex brain slices. The Journal of neuroscience : the official journal of the Society for Neuroscience, 1987. 7(4): p. 1223-49.

147. Kawai, M., et al., The relationship of amyloid plaques to cerebral capillaries in Alzheimer's disease. The American journal of pathology, 1990. 137(6): p. 143546.

148. Kidd, M., Paired helical filaments in electron microscopy of Alzheimer's disease. Nature, 1963. 197: p. 192-3.

149. Kidd, M., Alzheimer's Disease--an Electron Microscopical Study. Brain : a journal of neurology, 1964. 87: p. 307-20.

150. Knafo, S., et al., Pathology of synapses and dendritic spines. Neural plasticity, 2012. 2012: p. 972432.

151. Knowles, W.D., Normal anatomy and neurophysiology of the hippocampal formation. Journal of clinical neurophysiology : official publication of the American Electroencephalographic Society, 1992. 9(2): p. 252-63.

152. Koffie, R.M., et al., Oligomeric amyloid beta associates with postsynaptic densities and correlates with excitatory synapse loss near senile plaques. Proceedings of the National Academy of Sciences of the United States of America, 2009. 106(10): p. 4012-7.

153. Kopec, C.D., et al., Glutamate receptor exocytosis and spine enlargement during chemically induced long-term potentiation. The Journal of neuroscience : the official journal of the Society for Neuroscience, 2006. 26(7): p. 2000-9. 
154. Land, P.W. and L. Shamalla-Hannah, Experience-dependent plasticity of zinccontaining cortical circuits during a critical period of postnatal development. The Journal of comparative neurology, 2002. 447(1): p. 43-56.

155. Lang, C., et al., Transient expansion of synaptically connected dendritic spines upon induction of hippocampal long-term potentiation. Proceedings of the National Academy of Sciences of the United States of America, 2004. 101(47): p. 16665-70.

156. Lannfelt, L., et al., Safety, efficacy, and biomarker findings of PBT2 in targeting Abeta as a modifying therapy for Alzheimer's disease: a phase IIa, double-blind, randomised, placebo-controlled trial. Lancet neurology, 2008. 7(9): p. 779-86.

157. Larson, E.B., et al., Exercise is associated with reduced risk for incident dementia among persons 65 years of age and older. Annals of internal medicine, 2006. 144(2): p. 73-81.

158. Lavenex, P., P.B. Lavenex, and D.G. Amaral, Spatial relational learning persists following neonatal hippocampal lesions in macaque monkeys. Nature neuroscience, 2007. 10(2): p. 234-9.

159. Lawrence, J.J. and C.J. McBain, Interneuron diversity series: containing the detonation--feedforward inhibition in the CA3 hippocampus. Trends in neurosciences, 2003. 26(11): p. 631-40.

160. Lazarov, O., et al., Environmental enrichment reduces Abeta levels and amyloid deposition in transgenic mice. Cell, 2005. 120(5): p. 701-13.

161. Lee, J.Y., et al., Alteration of the cerebral zinc pool in a mouse model of Alzheimer disease. Journal of neuropathology and experimental neurology, 2012. 71(3): p. 211-22.

162. Lee, J.Y., et al., Contribution by synaptic zinc to the gender-disparate plaque formation in human Swedish mutant APP transgenic mice. Proceedings of the National Academy of Sciences of the United States of America, 2002. 99(11): p. 7705-10.

163. Lee, J.Y., et al., Dependence of the histofluorescently reactive zinc pool on zinc transporter-3 in the normal brain. Brain research, 2011. 1418: p. 12-22.

164. Lee, J.Y., et al., Essential role for zinc-triggered p75NTR activation in preconditioning neuroprotection. The Journal of neuroscience : the official journal of the Society for Neuroscience, 2008. 28(43): p. 10919-27.

165. Lee, J.Y., I. Mook-Jung, and J.Y. Koh, Histochemically reactive zinc in plaques of the Swedish mutant beta-amyloid precursor protein transgenic mice. The Journal of neuroscience : the official journal of the Society for Neuroscience, 1999. 19(11): p. RC10.

166. Lee, K.F., C. Soares, and J.C. Beique, Examining form and function of dendritic spines. Neural plasticity, 2012. 2012: p. 704103.

167. Lee, V.M., et al., A68: a major subunit of paired helical filaments and derivatized forms of normal Tau. Science, 1991. 251(4994): p. 675-8.

168. Leiby, C.C., 3rd, D.B. Bender, and C.M. Butter, Localization and detection of visual stimuli in monkeys with pulvinar lesions. Experimental brain research. Experimentelle Hirnforschung. Experimentation cerebrale, 1982. 48(3): p. 44954 .

169. Lendvai, B., et al., Experience-dependent plasticity of dendritic spines in the developing rat barrel cortex in vivo. Nature, 2000. 404(6780): p. 876-81.

170. Levy, E., et al., Mutation of the Alzheimer's disease amyloid gene in hereditary cerebral hemorrhage, Dutch type. Science, 1990. 248(4959): p. 1124-6. 
171. Li, Y., et al., Rapid translocation of Zn(2+) from presynaptic terminals into postsynaptic hippocampal neurons after physiological stimulation. Journal of neurophysiology, 2001. 86(5): p. 2597-604.

172. Liguz-Lecznar, M., et al., Dissociation of synaptic zinc level and zinc transporter 3 expression during postnatal development and after sensory deprivation in the barrel cortex of mice. Brain research bulletin, 2005. 66(2): p. 106-13.

173. Liu, S., et al., TLR2 is a primary receptor for Alzheimer's amyloid beta peptide to trigger neuroinflammatory activation. Journal of immunology, 2012. 188(3): p. 1098-107.

174. Liu, Y., et al., LPS receptor (CD14): a receptor for phagocytosis of Alzheimer's amyloid peptide. Brain : a journal of neurology, 2005. 128(Pt 8): p. 1778-89.

175. Lopes, J.P., et al., Galantamine potentiates the neuroprotective effect of memantine against NMDA-induced excitotoxicity. Brain and behavior, 2013. 3(2): p. 67-74.

176. Love, S., et al., Alz-50, ubiquitin and tau immunoreactivity of neurofibrillary tangles, Pick bodies and Lewy bodies. Journal of neuropathology and experimental neurology, 1988. 47(4): p. 393-405.

177. Lovell, M.A., et al., Copper, iron and zinc in Alzheimer's disease senile plaques. Journal of the neurological sciences, 1998. 158(1): p. 47-52.

178. Lue, L.F., et al., Inflammation, A beta deposition, and neurofibrillary tangle formation as correlates of Alzheimer's disease neurodegeneration. Journal of neuropathology and experimental neurology, 1996. 55(10): p. 1083-8.

179. Lund, J.S., et al., The origin of efferent pathways from the primary visual cortex, area 17, of the macaque monkey as shown by retrograde transport of horseradish peroxidase. The Journal of comparative neurology, 1975. 164(3): $\mathrm{p}$. 287-303.

180. Luth, H.J. and K. Brauer, The localization of AChE in the dorsal lateral geniculate nucleus of different mammals--a light and electron microscopical study. Acta histochemica, 1983. 72(2): p. 211-24.

181. Mandrekar-Colucci, S. and G.E. Landreth, Microglia and inflammation in Alzheimer's disease. CNS \& neurological disorders drug targets, 2010. 9(2): p. 156-67.

182. Marotta, C.A., R.E. Majocha, and B. Tate, Molecular and cellular biology of Alzheimer amyloid. Journal of molecular neuroscience : MN, 1992. 3(3): p. 11125.

183. Martel, G., et al., Zinc transporter $Z n T_{3}$ is involved in memory dependent on the hippocampus and perirhinal cortex. Behavioural brain research, 2011. 223(1): p. 233-8.

184. Martin, K.A. and D. Whitteridge, Form, function and intracortical projections of spiny neurones in the striate visual cortex of the cat. The Journal of physiology, 1984. 353: p. 463-504.

185. Masliah, E., et al., Late onset dementia with argyrophilic grains and subcortical tangles or atypical progressive supranuclear palsy? Annals of neurology, 1991. 29(4): p. 389-96.

186. Masliah, E., et al., Comparison of neurodegenerative pathology in transgenic mice overexpressing $V 717 F$ beta-amyloid precursor protein and Alzheimer's disease. The Journal of neuroscience : the official journal of the Society for Neuroscience, 1996. 16(18): p. 5795-811.

187. Masters, C.L., et al., Neuronal origin of a cerebral amyloid: neurofibrillary tangles of Alzheimer's disease contain the same protein as the amyloid of plaque cores and blood vessels. The EMBO journal, 1985. 4(11): p. 2757-63. 
188. Matsuzaki, M., et al., Dendritic spine geometry is critical for AMPA receptor expression in hippocampal CA1 pyramidal neurons. Nature neuroscience, 2001. 4(11): p. 1086-92.

189. Matsuzaki, M., et al., Structural basis of long-term potentiation in single dendritic spines. Nature, 2004. 429(6993): p. 761-6.

190. Matus, A., Growth of dendritic spines: a continuing story. Current opinion in neurobiology, 2005. 15(1): p. 67-72.

191. Mayeux, R. and Y. Stern, Epidemiology of Alzheimer disease. Cold Spring Harbor perspectives in medicine, 2012. 2(8).

192. McGeer, P.L. and E.G. McGeer, The inflammatory response system of brain: implications for therapy of Alzheimer and other neurodegenerative diseases. Brain research. Brain research reviews, 1995. 21(2): p. 195-218.

193. Mehta, A., et al., Excitotoxicity: bridge to various triggers in neurodegenerative disorders. European journal of pharmacology, 2013. 698(1-3): p. 6-18.

194. Mesulam, M.M., C. Geula, and M.A. Moran, Anatomy of cholinesterase inhibition in Alzheimer's disease: effect of physostigmine and tetrahydroaminoacridine on plaques and tangles. Annals of neurology, 1987. 22(6): p. 683-91.

195. Meyer-Luehmann, M., et al., Rapid appearance and local toxicity of amyloidbeta plaques in a mouse model of Alzheimer's disease. Nature, 2008. 451(7179): p. $720-4$.

196. Miao, J., et al., Cerebral microvascular amyloid beta protein deposition induces vascular degeneration and neuroinflammation in transgenic mice expressing human vasculotropic mutant amyloid beta precursor protein. The American journal of pathology, 2005. 167(2): p. 505-15.

197. Miller, L.M., et al., Synchrotron-based infrared and X-ray imaging shows focalized accumulation of $\mathrm{Cu}$ and $\mathrm{Zn}$ co-localized with beta-amyloid deposits in Alzheimer's disease. Journal of structural biology, 2006. 155(1): p. 30-7.

198. Mishkin, M. and L.G. Ungerleider, Contribution of striate inputs to the visuospatial functions of parieto-preoccipital cortex in monkeys. Behavioural brain research, 1982. 6(1): p. 57-77.

199. Miyashita, T., N. Ichinohe, and K.S. Rockland, Differential modes of termination of amygdalothalamic and amygdalocortical projections in the monkey. The Journal of comparative neurology, 2007. 502(2): p. 309-24.

200. Mocchegiani, E., L. Rink, and M. Blasco, Zinc and ageing (ZINCAGE Project). Biogerontology, 2006. 7(5-6): p. 305-6.

201. Morris, J.C., et al., Cerebral amyloid deposition and diffuse plaques in "normal" aging: Evidence for presymptomatic and very mild Alzheimer's disease.

Neurology, 1996. 46(3): p. 707-19.

202. Mrak, R.E., Microglia in Alzheimer brain: a neuropathological perspective. International journal of Alzheimer's disease, 2012. 2012: p. 165021.

203. Mullard, A., Sting of Alzheimer's failures offset by upcoming prevention trials. Nature reviews. Drug discovery, 2012. 11(9): p. 657-60.

204. Muly, E.C. and D. Fitzpatrick, The morphological basis for binocular and ON/OFF convergence in tree shrew striate cortex. The Journal of neuroscience : the official journal of the Society for Neuroscience, 1992. 12(4): p. 1319-34.

205. Mundel, P., et al., Synaptopodin: an actin-associated protein in telencephalic dendrites and renal podocytes. The Journal of cell biology, 1997. 139(1): p. 193204.

206. Nagappan, G., N.H. Woo, and B. Lu, Ama "zinc" link between TrkB transactivation and synaptic plasticity. Neuron, 2008. 57(4): p. 477-9. 
207. Nagele, R.G., et al., Contribution of glial cells to the development of amyloid plaques in Alzheimer's disease. Neurobiology of aging, 2004. 25(5): p. 663-74.

208. Nagerl, U.V., et al., Bidirectional activity-dependent morphological plasticity in hippocampal neurons. Neuron, 2004. 44(5): p. 759-67.

209. Nahmani, M. and A. Erisir, VGluT2 immunochemistry identifies thalamocortical terminals in layer 4 of adult and developing visual cortex. The Journal of comparative neurology, 2005. 484(4): p. 458-73.

210. Nakashima, A.S. and R.H. Dyck, Enhanced plasticity in zincergic, cortical circuits after exposure to enriched environments. The Journal of neuroscience : the official journal of the Society for Neuroscience, 2008. 28(51): p. 13995-9.

211. Ng, A.N. and H. Toresson, Gamma-secretase and metalloproteinase activity regulate the distribution of endoplasmic reticulum to hippocampal neuron dendritic spines. FASEB journal : official publication of the Federation of American Societies for Experimental Biology, 2008. 22(8): p. 2832-42.

212. Nicoll, R.A. and D. Schmitz, Synaptic plasticity at hippocampal mossy fibre synapses. Nature reviews. Neuroscience, 2005. 6(11): p. 863-76.

213. Palestini, M., et al., Glutamate, GABA, calbindin-D28k and parvalbumin immunoreactivity in the pulvinar-lateralis posterior complex of the cat: relation to the projection to the Clare-Bishop area. Neuroscience letters, 1993. 160(1): p. 89-92.

214. Palmiter, R.D., et al., ZnT-3, a putative transporter of zinc into synaptic vesicles. Proceedings of the National Academy of Sciences of the United States of America, 1996. 93(25): p. 14934-9.

215. Paoletti, P., P. Ascher, and J. Neyton, High-affinity zinc inhibition of NMDA NR1-NR2A receptors. The Journal of neuroscience : the official journal of the Society for Neuroscience, 1997. 17(15): p. 5711-25.

216. Paoletti, P., et al., Zinc at glutamatergic synapses. Neuroscience, 2009. 158(1): p. 126-36.

217. Paresce, D.M., R.N. Ghosh, and F.R. Maxfield, Microglial cells internalize aggregates of the Alzheimer's disease amyloid beta-protein via a scavenger receptor. Neuron, 1996. 17(3): p. 553-65.

218. Patel, N.C. and M.E. Bickford, Synaptic targets of cholinergic terminals in the pulvinar nucleus of the cat. The Journal of comparative neurology, 1997. 387(2): p. 266-78.

219. Perl, D.P., Neuropathology of Alzheimer's disease. The Mount Sinai journal of medicine, New York, 2010. 77(1): p. 32-42.

220. Perry, G., et al., Ubiquitin is detected in neurofibrillary tangles and senile plaque neurites of Alzheimer disease brains. Proceedings of the National Academy of Sciences of the United States of America, 1987. 84(9): p. 3033-6.

221. Peters, A. and I.R. Kaiserman-Abramof, The small pyramidal neuron of the rat cerebral cortex. The perikaryon, dendrites and spines. The American journal of anatomy, 1970. 127(4): p. 321-55.

222. Peters, A. and B.R. Payne, Numerical relationships between geniculocortical afferents and pyramidal cell modules in cat primary visual cortex. Cerebral cortex, 1993. 3(1): p. 69-78.

223. Pluta, R., et al., Sporadic Alzheimer's Disease Begins as Episodes of Brain Ischemia and Ischemically Dysregulated Alzheimer's Disease Genes. Molecular neurobiology, 2013.

224. Powell, T.P. and A.E. Hendrickson, Similarity in number of neurons through the depth of the cortex in the binocular and monocular parts of area 17 of the monkey. Brain research, 1981. 216(2): p. 409-13. 
225. Price, J.L., et al., The distribution of tangles, plaques and related immunohistochemical markers in healthy aging and Alzheimer's disease. Neurobiology of aging, 1991. 12(4): p. 295-312.

226. Prusky, G.T. and R.M. Douglas, Characterization of mouse cortical spatial vision. Vision research, 2004. 44(28): p. 3411-8.

227. Qian, J. and J.L. Noebels, Visualization of transmitter release with zinc fluorescence detection at the mouse hippocampal mossy fibre synapse. The Journal of physiology, 2005. 566(Pt 3): p. 747-58.

228. Quiroz-Baez, R., D. Flores-Dominguez, and C. Arias, Synaptic Aging is Associated with Mitochondrial Dysfunction, Reduced Antioxidant Contents and Increased Vulnerability to Amyloid-beta Toxicity. Current Alzheimer research, 2013. 10(3): p. 324-31.

229. Rachline, J., et al., The micromolar zinc-binding domain on the NMDA receptor subunit NR2B. The Journal of neuroscience : the official journal of the Society for Neuroscience, 2005. 25(2): p. 308-17.

230. Ramsden, M., et al., Age-dependent neurofibrillary tangle formation, neuron loss, and memory impairment in a mouse model of human tauopathy (P3O1L). The Journal of neuroscience : the official journal of the Society for Neuroscience, 2005. 25(46): p. 10637-47.

231. Reddy, P.H. and M.F. Beal, Amyloid beta, mitochondrial dysfunction and synaptic damage: implications for cognitive decline in aging and Alzheimer's disease. Trends in molecular medicine, 2008. 14(2): p. 45-53.

232. Reddy, P.H., et al., Amyloid-beta and mitochondria in aging and Alzheimer's disease: implications for synaptic damage and cognitive decline. Journal of Alzheimer's disease : JAD, 2010. 20 Suppl 2: p. S499-512.

233. Reddy, P.H., et al., Differential loss of synaptic proteins in Alzheimer's disease: implications for synaptic dysfunction. Journal of Alzheimer's disease : JAD, 2005. 7(2): p. 103-17; discussion 173-80.

234. Ritchie, C.W., et al., Metal-protein attenuation with iodochlorhydroxyquin (clioquinol) targeting Abeta amyloid deposition and toxicity in Alzheimer disease: a pilot phase 2 clinical trial. Archives of neurology, 2003. 60(12): p. 1685-91.

235. Rochefort, N.L. and A. Konnerth, Dendritic spines: from structure to in vivo function. EMBO reports, 2012. 13(8): p. 699-708.

236. Rockenstein, E.M., et al., Levels and alternative splicing of amyloid beta protein precursor (APP) transcripts in brains of APP transgenic mice and humans with Alzheimer's disease. The Journal of biological chemistry, 1995. 270(47): p. 28257-67.

237. Savva, G.M., et al., Age, neuropathology, and dementia. The New England journal of medicine, 2009. 360(22): p. 2302-9.

238. Schaeffer, E.L., M. Figueiro, and W.F. Gattaz, Insights into Alzheimer disease pathogenesis from studies in transgenic animal models. Clinics, 2011. 66 Suppl 1: p. 45-54.

239. Schneider, J.A., et al., Subcortical infarcts, Alzheimer's disease pathology, and memory function in older persons. Annals of neurology, 2007. 62(1): p. 59-66.

240. Schuster, T., M. Krug, and J. Wenzel, Spinules in axospinous synapses of the rat dentate gyrus: changes in density following long-term potentiation. Brain research, 1990. 523(1): p. 171-4.

241. Segal, M., Dendritic spines and long-term plasticity. Nature reviews. Neuroscience, 2005. 6(4): p. 277-84. 
242. Segal, M., A. Vlachos, and E. Korkotian, The spine apparatus, synaptopodin, and dendritic spine plasticity. The Neuroscientist : a review journal bringing neurobiology, neurology and psychiatry, 2010. 16(2): p. 125-31.

243. Selkoe, D.J., Alzheimer's disease: genes, proteins, and therapy. Physiological reviews, 2001. 81(2): p. 741-66.

244. Sensi, S.L., et al., Zinc in the physiology and pathology of the CNS. Nature reviews. Neuroscience, 2009. 10(11): p. 780-91.

245. Sensi, S.L., et al., The neurophysiology and pathology of brain zinc. The Journal of neuroscience : the official journal of the Society for Neuroscience, 2011. 31(45): p. 16076-85.

246. Sierra, A., et al., Janus-faced microglia: beneficial and detrimental consequences of microglial phagocytosis. Frontiers in cellular neuroscience, 2013. 7: p. 6.

247. Smyth, K.A., et al., Worker functions and traits associated with occupations and the development of $A D$. Neurology, 2004. 63(3): p. 498-503.

248. Solito, E. and M. Sastre, Microglia function in Alzheimer's disease. Frontiers in pharmacology, 2012. 3: p. 14.

249. Soriano, E. and M. Frotscher, Spiny nonpyramidal neurons in the CA3 region of the rat hippocampus are glutamate-like immunoreactive and receive convergent mossy fiber input. The Journal of comparative neurology, 1993. 333(3): p. 435-48.

250. Spacek, J. and K.M. Harris, Three-dimensional organization of cell adhesion junctions at synapses and dendritic spines in area CA1 of the rat hippocampus. The Journal of comparative neurology, 1998. 393(1): p. 58-68.

251. Sperling, R., et al., Amyloid-related imaging abnormalities in patients with Alzheimer's disease treated with bapineuzumab: a retrospective analysis. Lancet neurology, 2012. 11(3): p. 241-9.

252. Spires-Jones, T. and S. Knafo, Spines, plasticity, and cognition in Alzheimer's model mice. Neural plasticity, 2012. 2012: p. 319836.

253. Spires-Jones, T.L., et al., Impaired spine stability underlies plaque-related spine loss in an Alzheimer's disease mouse model. The American journal of pathology, 2007. 171(4): p. 1304-11.

254. Spires-Jones, T.L., et al., Passive immunotherapy rapidly increases structural plasticity in a mouse model of Alzheimer disease. Neurobiology of disease, 2009. 33(2): p. 213-20.

255. Stalder, M., et al., $3 D$-Reconstruction of microglia and amyloid in APP23 transgenic mice: no evidence of intracellular amyloid. Neurobiology of aging, 2001. 22(3): p. 427-34.

256. Stalder, M., et al., Association of microglia with amyloid plaques in brains of APP23 transgenic mice. The American journal of pathology, 1999. 154(6): p. 1673-84.

257. Stepniewska, I., T.M. Preuss, and J.H. Kaas, Architectonics, somatotopic organization, and ipsilateral cortical connections of the primary motor area (M1) of owl monkeys. The Journal of comparative neurology, 1993 330(2): p. 238-71.

258. Steward, O. and S.A. Scoville, Cells of origin of entorhinal cortical afferents to the hippocampus and fascia dentata of the rat. The Journal of comparative neurology, 1976. 169(3): p. 347-70.

259. Stoltenberg, M., et al., Amyloid plaques arise from zinc-enriched cortical layers in APP/PS1 transgenic mice and are paradoxically enlarged with dietary zinc deficiency. Neuroscience, 2007. 150(2): p. 357-69. 
260. Storey, E., K. Beyreuther, and C.L. Masters, Alzheimer's disease amyloid precursor protein on the surface of cortical neurons in primary culture colocalizes with adhesion patch components. Brain research, 1996. 735(2): p. 21731.

261. Streit, W.J., An improved staining method for rat microglial cells using the lectin from Griffonia simplicifolia (GSA I-B4). The journal of histochemistry and cytochemistry : official journal of the Histochemistry Society, 1990. 38(11): p. 1683-6.

262. Streit, W.J. and G.W. Kreutzberg, Lectin binding by resting and reactive microglia. Journal of neurocytology, 1987. 16(2): p. 249-60.

263. Tackenberg, C. and R. Brandt, Divergent pathways mediate spine alterations and cell death induced by amyloid-beta, wild-type tau, and R406W tau. The Journal of neuroscience : the official journal of the Society for Neuroscience, 2009. 29(46): p. 14439-50.

264. Takahashi, R.H., et al., Alzheimer beta-amyloid peptides: normal and abnormal localization. Histology and histopathology, 2002. 17(1): p. 239-46.

265. Takeda, A., Zinc homeostasis and functions of zinc in the brain. Biometals : an international journal on the role of metal ions in biology, biochemistry, and medicine, 2001. 14(3-4): p. 343-51.

266. Tanaka, T., M. Ueno, and T. Yamashita, Engulfment of axon debris by microglia requires p38 MAPK activity. The Journal of biological chemistry, 2009. 284(32): p. 21626-36.

267. Tao-Cheng, J.H., et al., Rapid turnover of spinules at synaptic terminals. Neuroscience, 2009. 160(1): p. 42-50.

268. Tarrant, S.B. and A. Routtenberg, The synaptic spinule in the dendritic spine: electron microscopic study of the hippocampal dentate gyrus. Tissue \& cell, 1977. 9(3): p. 461-73.

269. Terry, R.D., The Fine Structure of Neurofibrillary Tangles in Alzheimer's Disease. Journal of neuropathology and experimental neurology, 1963. 22: p. 629-42.

270. Terry, R.D., N.K. Gonatas, and M. Weiss, Ultrastructural Studies in Alzheimer's Presenile Dementia. The American journal of pathology, 1964. 44: p. 269-97.

271. Terry, R.D., et al., Physical basis of cognitive alterations in Alzheimer's disease: synapse loss is the major correlate of cognitive impairment. Annals of neurology, 1991. 30(4): p. 572-80.

272. Thal, D.R., et al., The development of amyloid beta protein deposits in the aged brain. Science of aging knowledge environment : SAGE KE, 2006. 2006(6): p. re1.

273. Thal, D.R., et al., Vascular pathology in Alzheimer disease: correlation of cerebral amyloid angiopathy and arteriosclerosis/lipohyalinosis with cognitive decline. Journal of neuropathology and experimental neurology, 2003. 62(12): p. 1287-301.

274. Thal, D.R., et al., Phases of A beta-deposition in the human brain and its relevance for the development of $A D$. Neurology, 2002. 58(12): p. 1791-800.

275. Thal, D.R., et al., Sequence of Abeta-protein deposition in the human medial temporal lobe. Journal of neuropathology and experimental neurology, 2000. 59(8): p. 733-48.

276. Thind, K. and M.N. Sabbagh, Pathological correlates of cognitive decline in Alzheimer's disease. Panminerva medica, 2007. 49(4): p. 191-5.

277. Tomlinson, B.E., G. Blessed, and M. Roth, Observations on the brains of nondemented old people. Journal of the neurological sciences, 1968. 7(2): p. 331-56. 
278. Tougu, V., et al., Zn(II)- and Cu(II)-induced non-fibrillar aggregates of amyloidbeta (1-42) peptide are transformed to amyloid fibrils, both spontaneously and under the influence of metal chelators. Journal of neurochemistry, 2009. 110(6): p. 1784-95.

279. Tyas, S.L., et al., Healthy ageing in the Nun Study: definition and neuropathologic correlates. Age and ageing, 2007. 36(6): p. 650-5.

280. Tycko, R., et al., Evidence for novel beta-sheet structures in Iowa mutant betaamyloid fibrils. Biochemistry, 2009. 48(26): p. 6072-84.

281. Ungerleider, L.G., Functional brain imaging studies of cortical mechanisms for memory. Science, 1995. 270(5237): p. 769-75.

282. Valenzuela, M.J., et al., Multiple biological pathways link cognitive lifestyle to protection from dementia. Biological psychiatry, 2012. 71(9): p. 783-91.

283. Van Nostrand, W.E., et al., Pathogenic effects of D23N Iowa mutant amyloid beta -protein. The Journal of biological chemistry, 2001. 276(35): p. 32860-6.

284. Venkitaramani, D.V., et al., Beta-amyloid modulation of synaptic transmission and plasticity. The Journal of neuroscience : the official journal of the Society for Neuroscience, 2007. 27(44): p. 11832-7.

285. Verret, L., et al., Transient enriched housing before amyloidosis onset sustains cognitive improvement in Tg2576 mice. Neurobiology of aging, 2013. 34(1): p. 211-25.

286. Vida, I. and M. Frotscher, A hippocampal interneuron associated with the mossy fiber system. Proceedings of the National Academy of Sciences of the United States of America, 2000. 97(3): p. 1275-80.

287. Vinters, H.V., Cerebral amyloid angiopathy. A critical review. Stroke; a journal of cerebral circulation, 1987. 18(2): p. 311-24.

288. Vlachos, A., Synaptopodin and the spine apparatus organelle-regulators of different forms of synaptic plasticity? Annals of anatomy = Anatomischer Anzeiger : official organ of the Anatomische Gesellschaft, 2012. 194(4): p. 31720.

289. Vlachos, A., et al., Synaptopodin regulates plasticity of dendritic spines in hippocampal neurons. The Journal of neuroscience : the official journal of the Society for Neuroscience, 2009. 29(4): p. 1017-33.

290. Wang, K.C., et al., Oligodendrocyte-myelin glycoprotein is a Nogo receptor ligand that inhibits neurite outgrowth. Nature, 2002. 417(6892): p. 941-4.

291. Wegiel, J., et al., Microglia cells are the driving force in fibrillar plaque formation, whereas astrocytes are a leading factor in plague degradation. Acta neuropathologica, 2000. 100(4): p. 356-64.

292. Wei, W., et al., Amyloid beta from axons and dendrites reduces local spine number and plasticity. Nature neuroscience, 2010. 13(2): p. 190-6.

293. Wilson, R.S., et al., Cognitive activity and incident $A D$ in a population-based sample of older persons. Neurology, 2002. 59(12): p. 1910-4.

294. Wiltfang, J., et al., Highly conserved and disease-specific patterns of carboxyterminally truncated Abeta peptides 1-37/38/39 in addition to 1-40/42 in Alzheimer's disease and in patients with chronic neuroinflammation. Journal of neurochemistry, 2002. 81(3): p. 481-96.

295. Wirths, O., et al., Axonopathy in an APP/PS1 transgenic mouse model of Alzheimer's disease. Acta neuropathologica, 2006. 111(4): p. 312-9.

296. Wisniewski, H.M., H.K. Narang, and R.D. Terry, Neurofibrillary tangles of paired helical filaments. Journal of the neurological sciences, 1976. 27(2): p. 17381. 
297. Wisniewski, H.M. and R.D. Terry, Morphology of the aging brain, human and animal. Progress in brain research, 1973. 40(o): p. 167-86.

298. Xu, F., et al., Early-onset subicular microvascular amyloid and neuroinflammation correlate with behavioral deficits in vasculotropic mutant amyloid beta-protein precursor transgenic mice. Neuroscience, 2007. 146(1): p. 98-107.

299. Yamaguchi, H., et al., Alzheimer type dementia: diffuse type of senile plaques demonstrated by beta protein immunostaining. Progress in clinical and biological research, 1989. 317: p. 467-74.

30o. Yamaguchi, H., et al., Amyloid beta protein (Abeta) starts to deposit as plasma membrane-bound form in diffuse plaques of brains from hereditary cerebral hemorrhage with amyloidosis-Dutch type, Alzheimer disease and nondemented aged subjects. Journal of neuropathology and experimental neurology, 2000. 59(8): p. 723-32.

301. Yang, Y., et al., Spine expansion and stabilization associated with long-term potentiation. The Journal of neuroscience : the official journal of the Society for Neuroscience, 2008. 28(22): p. 5740-51.

302. Yuan, Q., et al., Existence of different types of senile plaques between brain and spinal cord of TgCRND8 mice. Neurochemistry international, 2013. 62(3): p. 211-20.

303. Yuste, R., A. Majewska, and K. Holthoff, From form to function: calcium compartmentalization in dendritic spines. Nature neuroscience, 2000. 3(7): p. 653-9.

304. Zhou, Q., K.J. Homma, and M.M. Poo, Shrinkage of dendritic spines associated with long-term depression of hippocampal synapses. Neuron, 2004. 44(5): p. 749-57. 


\section{CURRICULUM VITAE}

\section{Dmitry Familtsev}

\section{Personal Information}

Home address: $\quad 2108$ Biljana Drive apt 9

Louisville, KY 40206

Preferred Phone: $\quad 5028079514$

E-mail: $\quad$ familtsev@gmail.com

DOB: $\quad$ September, 5,1985

\section{$\underline{\text { Education }}$}

Graduate (Ph.D.) 2008-present Anatomical Sciences and Neurobiology Department of Medicine, University of Louisville, Louisville, KY

(Non-terminal M.S. awarded in 2010)

Graduate (M.D.) ～2002-2008

Omsk State Medical academy, Omsk, Russia

\section{Grants and Fellowships}

IPIBS Fellowship 2008-2010

\section{Honors and Awards}

Medical School 2008

Omsk State Medical Academy, Omsk,

Diploma with Honors Russia 


\section{Professional Affiliations}

Society for Neuroscience 20012-present

\section{Publications}

Poster presentations and Abstracts

Familtsev D., Bickford, M., Friedland R.. (2012, October). Zinc, synaptopodin and Alzheimer's disease. Poster presented at: Neuroscience 2012; New Orleans, LA

Harrison B., Familtsev D., Hougland M., Moon L., Petruska J.. (2009, June). Comparative Analyses of Molecular Signaling During Distinct Modes of Axonal Plasticity In Vivo: Collateral Sprouting vs. Regeneration. Poster presented at: 15th Annual Kentucky Spinal Cord and Head Injury Research Trust Symposium; Louisville, $\mathrm{KY}$

Harrison B., Hargrove C., Guenthner J., Familtsev D., Hougland M., Moon L., Petruska J.. (2009, October). Comparative Analyses of Molecular Signaling During Distinct Modes of Axonal Plasticity in Vivo: Collateral Sprouting vs. Regeneration. Poster presented at: Neuroscience 2009; Chicago, IL 